\title{
Wissensgenese 2.0: Social-Media-Analysen
}

Digitalisierung oder auch „digitale Transformation“ handelt der öffentliche Diskurs seit geraumer Zeit als das Phänomen, das die Gegenwart in nahezu jeder Hinsicht prägt und ebenso unsere Zukunft wesentlich mitbestimmen wird. Die Google-Gesellschaft und Das Wikipedia-Universum wurden ausgerufen und damit einhergehend vom digitalen Wandel des Wissens berichtet oder auch danach gefragt, wie das Internet unsere Wissenskultur verändert (Lehmann, \& Schetsche, 2007; Pscheida, 2010). ${ }^{1}$

Da der digitale Wandel keinen Gesellschaftsbereich unberührt lässt, erstreckt er sich auch auf die zuvor thematisierte wissenssoziologische Organisationsforschung. Etliche Organisationen, nicht zuletzt deutsche Automobilhersteller, haben sich schon längst auf den Weg in Richtung einer Industrie 4.0 gemacht; nun stehen sie auch einem Wandel in Bezug auf die Entstehung und Erschließung von Wissen gegenüber, und dieser ist vor allem durch das Web 2.0 und Social Media bedingt (Ebel, \& Hofer, 2014; Köhler, \& Wollschläger, 2014).

Im Jahr 1992 prägte Edward Barrett den Begriff „Sociomedia“ und erkannte schon damals:

In essence, then, "sociomedia" signifies that when we design computer media we are hardwiring a mechanism for the social construction of knowledge (Barrett, 1992, S. 1).

Sabine Maasen (2012) erklärt Barretts Befund dahingehend, dass die „soziomediale Wissensarena“ als „extended conversation among knowledgeable peers“

\footnotetext{
${ }^{1}$ Lehmann und Schetsche (2007) bieten mit ihrem Sammelband Die Google-Gesellschaft. Vom digitalen Wandel des Wissens eine Bestandsaufnahme der verschiedenen Dimensionen der „,internetbasierten Wissensgesellschaft“. Pscheida (2010) betrachtet Das WikipediaUniversum. Wie das Internet unsere Wissenskultur verändert und diskutiert das ,,wissenskulturreformerische Potential des Internets".
} 
$\mathrm{zu}$ verstehen ist; diese verbinde die individuellen Nutzer sozialer Medien zu einem „Netzwerk von Wissensproduzenten und Wissenskonsumenten“ vor neuen Raum- und Zeithorizonten. Ähnlich fassen Guendalina Graffigna und Giuseppe Riva (2015) Social Media und titulieren diese als ,the arena of interpersonal knowledge and practice sharing“. Es handle sich dabei um fruchtbaren Boden für den Austausch von Wissen, Informationen, ,Klatsch und Tratsch“ und Bewertungen; diesem komme große Bedeutung für den Vergleich individueller Erfahrungen und die Orientierung in zwischenmenschlichen Entscheidungsprozessen zu. Graffigna und Riva begreifen Social Media als eine bedeutsame Wissens- und Unterstützungs-Ressource für Individuen (Graffigna, \& Riva, 2015; Maasen, 2012, S. 65). ${ }^{2}$

Den mit der Digitalisierung des Wissens einhergehenden Prozessen hat sich auch Nina Degele (2007) gewidmet und eine These der Informierung des Wissens aufgestellt:

Die These der Informierung von Wissen behauptet also, dass der Einsatz von Computern Wissen in eine neue, nämlich inhaltsarme und dafür verarbeitungs- und inszenierungsfreundliche Form bringt: Wissen zweiter Ordnung wird wichtiger als inhaltliches Domänenwissen, als Wissen darüber, wie inhaltsspezifisches Domänenwissen zu organisieren, zu inszenieren und in Aktion zu bringen ist. Grund: Inhaltliches Wissen verliert an Bedeutung, denn es veraltet zu schnell und es wird zuviel (Degele, 2007, S. 396).

Degele konstatiert einen Wandel des Wissens hin zu einem neuen Typus, welchen sie ,informiertes Wissen“ nennt; dabei erkennt sie eine Verlagerung weg von inhaltlichen und hin zu nicht-inhaltlichen Komponenten (Maasen, 2012, S. 86). In Bezug auf Degeles These bemerkt Maasen:

Die Informatisierung des Wissens hat Rückwirkungen auf Wissen 1. Ordnung (know that) wie auf Wissen 2. Ordnung (know how). Degele selbst diagnostiziert eine Verschiebung in Richtung auf Wissen 2. Ordnung: knowing how genießt zunehmend Priorität gegenüber knowing that - zumal die Wissensbestände sinkende Halbwertzeiten verzeichnen. Dies lässt sich radikalisieren: Die Produktion von Wissensbeständen ist immer weniger von den technischen Medien seiner Genese, Anwendung und Verwaltung zu trennen (Maasen, 2012, S. 86 f., Hervorheb. i. O.).

Einer solchen Digitalisierung des Wissens steht auch die deutsche Automobilindustrie gegenüber. Die Unternehmen gewinnen, wie zuvor dargelegt, das

${ }^{2}$ Social Media for Knowledge Sharing in Automtovive Repair hat aus wirtschaftswissenschaftlicher Perspektive Finkbeiner (2017) untersucht. 
für sie relevante Wissen, das insbesondere im Kundenwissen besteht, bislang in erster Linie durch klassische Marktforschung. Für Organisationen bedeutsames Wissen ist heute vor allem auch in sozialen Medien enthalten und kann beispielsweise durch Online-Forschung untersucht werden; eine Methode, die angewendet werden kann, sind Social-Media-Analysen. Die Erzeugung von organisationalem Wissen im Kontext des digitalen Wandels unter Rückgriff auf das Web 2.0 beziehungsweise soziale Medien thematisiert das vorliegende Kapitel als Wissensgenese 2.0: Social-Media-Analysen.

Allerdings sollte - dies ist vorwegzunehmen - nicht von Kundenwissen, das in Social Media enthalten ist, ausgegangen werden, sondern von Nutzerwissen. Technische, vor allem aber datenschutzrechtliche Restriktionen erlauben derzeit keine verlässlichen Rückschlüsse von über soziale Medien veröffentlichen Botschaften auf die Identität ihrer Sender; die Nutzer von sozialen Medien bleiben der Online-Forschung gegenüber unbekannt, Inhalte von Social Media werden anonym publiziert. Aus diesem Grund ist auch nicht bekannt, ob Nutzer sozialer Medien Kunden bestimmter Unternehmen sind. Entsprechend nimmt auch Frauke Zeller (2017) ihre methodologischen Überlegungen zu Social-Media-Analysen auf: ${ }^{3}$

Linking social media data and other data sources can be regarded as a challenging and, at the same time, redeeming feature. It is challenging given the complexity of the data available online, and the research design needed for a sound research project analyzing multiple data formats and/or sources. The redeeming feature can be seen, for example, in the availability of vast quantities of different kinds of social media data, which help to contextualize findings and can potentially provide a work-around strategy to the anonymity challenge of online data. Social scientists are primarily interested in understanding social interaction embedded in our social world or context. This means we need to be able to contextualize data with the respective social world (usually demographic and other information). This is difficult - if not almost impossible given that anyone can pretend to be anybody on social media. Linking social media data and other data sources can help by enriching the results, and providing additional information (Zeller, 2017b, S. 386).

\footnotetext{
${ }^{3}$ Einen Überblick über die sozialwissenschaftliche Online-Forschung geben die zahlreichen Beiträge in dem von Welker, Taddicken, Schmidt und Jackob herausgegebenen Handbuch Online-Forschung. Sozialwissenschaftliche Datengewinnung und-auswertung in digitalen Netzen (2014). Die Artikel in dem Sammelband Digital Methods for Social Science. An Interdisciplinary Guide to Research Innovation (2016) von Snee, Hine, Morey, Roberts und Watson beleuchten verschiedene digitale Methoden für die Sozialwissenschaften mit heterogenem Fokus.
} 
Trotz mancher Herausforderungen bergen Social-Media-Analysen also auch erhebliche Potentiale. Grundlegende Herausforderungen einer empirischen Forschung mit sozialen Medien entsprechen, so Zeller, folgenden Merkmalen der Online-Forschung:

Flüchtigkeit/Dynamik der Inhalte, Multimedialität/Heterogenität der Inhalte, Nonlinearität/Hypertextualität, Reaktivität und Personalisierung, Digitalisierung und Maschinenlesbarkeit, Qualität (Zeller, 2017a, S. 402).

Hinzu kommen Schwierigkeiten, die auf der Besonderheit sozialer Medien beruhen. Danah Boyd und Kate Crawford diskutieren insgesamt sechs Kritikpunkte in Bezug auf Big Data, die ebenso auf Social Media übertragbar sind. Dies geschieht derart prägnant, dass ihre Kritik den weiteren in diesem Kapitel behandelten Themen vorangestellt wird. (1) Boyd und Crawford üben Kritik daran, dass die Social-Media-Forschung die Definition von Wissen verändert:

Big Data creates a radical shift in how we think about research. [... ] Big Data reframes key questions about the constitution of knowledge, the processes of research, how we should engage with information, and the nature and the categorization of reality (Boyd, \& Crawford, 2012, S. 665).

Diesen Wandel der Wissensdefinition sehen Boyd und Crawford auch im Einsatz von Software beziehungsweise deren Einfluss auf die Social-Media-Forschung begründet:

If we are observing the automation of particular kinds of research functions, then we must consider the inbuilt flaws of the machine tools (Boyd, \& Crawford, 2012, S. 666).

(2) Sie mahnen die quantitative Ausrichtung der Social-Media-Forschung und auch die Fehleinschätzung deren höheren Potentials im Vergleich zu qualitativen Studien an:

Claims to objectivity and accuracy are misleading. [... ] Big Data offers the humanistic disciplines a new way to claim the status of quantitative science and objective method. It makes many more social spaces quantifiable. In reality, working with Big Data is still subjective, and what it quantifies does not necessarily have a closer claim on objective truth - particularly when considering messages from social media sites. But there remains a mistaken belief that qualitative researchers are in the business of interpreting stories and quantitative researchers are in the business of producing facts (Boyd, \& Crawford, 2012, S. 666 f.). 
Für Boyd und Crawford besteht der Kern einer Datenanalyse in der Interpretation der Daten; diese unterlägen jedoch sowohl Einschränkungen als auch Verzerrungen:

Interpretation is at the center of data analysis. Regardless of the size of a data, it is subject to limitation and bias. Without those biases and limitations being understood and outlined, misinterpretation is the result. Data analysis is most effective when researchers take account of the complex methodological processes that underlie the analysis of that data (Boyd, \& Crawford, 2012, S. 668).

(3) Ihre Kritik knüpft an die Kontrastierung quantitativer und qualitativer Methoden an und bezieht sich auf die Datenqualität:

Bigger data are not always better data (Boyd, \& Crawford, 2012, S. 668).

Zum einen könne anhand der oft hohen Quantität der über soziale Medien gewonnenen Daten nicht auch auf deren Repräsentativität geschlossen werden; zum anderen sei das Potential von Small Data nicht zu unterschätzen. (4) Boyd und Crawford thematisieren die Bedeutung des Interpretationsprozesses im Rahmen von Social-Media-Analysen und halten folgende wesentliche Erkenntnis fest:

Taken out of context, Big Data loses its meaning (Boyd, \& Crawford, 2012, S. 670).

Eine Untersuchung von Social-Media-Daten ist nach Ansicht von Boyd und Crawford nur unter Einbezug von deren Kontext wertvoll; aus ihrem Zusammenhang gerissen verlören die Daten jegliche Bedeutung. Bei Zeller liest sich das so:

Die Vorstellung also, dass Zahlen für sich sprächen, ist bestenfalls naiv oder auch schlichtweg falsch (Zeller, 2017a, S. 403).

(5) Ein Kritikpunkt greift ethische Aspekte auf:

Just because it is accessible does not make it ethical (Boyd, \& Crawford, 2012, S. 671).

(6) Boyd und Crawford beanstanden den reglementierten Zugang, den die Wissenschaft beziehungsweise die wissenschaftliche Forschung im Vergleich mit Unternehmen zu sozialen Medien hat:

Limited access to Big Data creates new digital divides (Boyd, \& Crawford, 2012, S. 673). 
Unter Rückgriff auf Boyd und Crawford sind zentrale Punkte der an der SocialMedia-Forschung und damit der Online-Forschung geübten Kritik genannt. Es bleibt auf eine weitere Besonderheit der Social-Media-Forschung hinzuweisen: Soziale Medien können sowohl Gegenstand als auch Instrument der Forschung sein. Im Fokus der vorliegenden Arbeit stehen Social-Media-Analysen und damit auch soziale Medien als ein neues und mächtiges Instrument sozialwissenschaftlicher Forschung, das verantwortungsvolles Handeln erfordert (König, Stahl, \& Wiegand, 2014; Welker, \& Kloß, 2014).

Im Hinblick auf den digitalen Wandel der Entstehung und Erschließung von Wissen kommt dieser Methode eine nicht zu unterschätzende Bedeutung zu, die das vorliegende Kapitel in den Blick nimmt. Konkret geschieht dies in sechs Schritten. Zunächst wird geklärt, was überhaupt unter dem Web 2.0 und sozialen Medien zu verstehen ist und wie diese Phänomene die Gegenwart, insbesondere auch die von Organisationen prägen. Im Anschluss daran geht es um die sich etablierenden Computational Social Sciences und Digital Humanities als interdisziplinären Zusammenschluss von Sozial- oder auch Geisteswissenschaften mit der Informatik, der sich mit Online-Forschung und Big Data befasst. Anknüpfend an die schon thematisierte Kritik von Boyd und Crawford wird auf methodologische Besonderheiten und ethische Aspekte der Methode eingegangen. Ein weiterer Abschnitt behandelt die methodische Umsetzung von Social-Media-Analysen und bildet deren Status quo ab. Um die Relevanz der Methode über unterschiedliche Disziplinen hinweg und auch in ihrer Vielseitigkeit zu zeigen, werden Social-Media-Analysen sodann aus weiteren Blickrichtungen vorgestellt. Zum Abschluss stehen die diversen Einsatzgebiete von Social-Media-Analysen im Fokus der Betrachtung, wobei der Schwerpunkt auf der Marktforschung im Web 2.0 liegt. $^{4}$

\footnotetext{
${ }^{4}$ Es ist darauf hinzuweisen, dass mittlerweile eine Unmenge an Literatur über soziale Medien und der Forschung mit und zu sozialen Medien vorliegt. Wie sich im Verlauf dieses Kapitels zeigen wird, sind es nicht nur die Sozialwissenschaften, die sich mit dem Thema beschäftigen; Forschungsfragen rund um soziale Medien werden disziplinübergreifend und interdisziplinär verfolgt. Aus forschungsökonomischen und -strategischen Gründen war es unmöglich, allen Beiträgen die verdiente Beachtung zu schenken; es galt zu selektieren. Dennoch macht diese Forschungsarbeit auf den von König, Stahl und Wiegand (2014) publizierten Sammelband Soziale Medien. Gegenstand und Instrument der Forschung sowie auf die Reihe Neue Schriften zur Online-Forschung und hier insbesondere auf den zweibändigen Sammelband Kommunikation, Partizipation und Wirkungen im Social Web von Zerfass, Welker und Schmidt (2008a, 2008b) aufmerksam. Diese stellen eine gute Ausgangsbasis für den Einstieg in die sozialwissenschaftliche Beschäftigung mit Social Media als einem Gegenstand oder Instrument der Forschung dar. Einen Überblick über die Herausforderungen, die soziale Medien für die Forschung bedeuten, geben mit ihrem Social Media Honeycomb Model ferner Kietzmann, Silvestre, McCarthy und Pitt (2012).
} 


\subsection{Social Media im Web 2.0}

The Internet is now an established technology that has evolved rapidly in terms of its availability and diversity of uses. The Internet is most easily understood from a technological perspective as a global network of millions of computers (mobile or fixed platforms) connected through a set of protocols and a broad array of electronic, wireless and optical networking technologies. [. . ] the Internet now serves as a basic infrastructure that supports an increasingly extensive range of information resources and services. The World Wide Web constitutes one of the most popular and widely used services running on the Internet (Sudulich, Wall, Gibson, Cantijoch, \& Ward, 2014, S. 5 f.).

In den letzten 20 Jahren hat sich das „Universalmedium Internet“ rasant entwickelt und gesellschaftlich etabliert. „Social Media“ oder auch „soziale Medien“, zwei Begriffe, die das gleiche Phänomen benennen und daher in dieser Arbeit synonym gebraucht werden, sind in den Fokus der öffentlichen wie wissenschaftlichen Aufmerksamkeit gerückt. Soziale Medien bilden heutzutage ein enormes Konstrukt digital vernetzter Kommunikation, das sich aus heterogenen Bausteinen zusammensetzt, zum Beispiel Netzwerkplattformen wie Facebook, Multimediaplattformen wie YouTube oder auch Microblogs wie Twitter. Es ist die Rede von der „Kommunikationsrevolution Social Media“ (Ehlers, 2013; Kaplan, \& Haenlein, 2012; McCay-Peet, \& Quan-Haase, 2017, S. 13 ff.; Taddicken, \& Schmidt, 2017, S. 4; Schmidt, 2018, S. 7 ff.).

Mittlerweile kann sich der Begriff „Social Media“ in eine Riege fast schon inflationär verwendeter Begriffe, zu denen auch „Big Data“ gehört, einreihen. „Social Media“ kommt Schlagwortcharakter zu; dies bedeutet auch, dass der Begriff inzwischen fast jede Klarheit eingebüßt hat. Dies macht eine Auseinandersetzung mit der Frage, was sich hinter dem Phänomen ,soziale Medien“ verbirgt, unerlässlich (Alpar, \& Blaschke, 2008, S. 4; Scheffler, 2014, S. 13; Taddicken, \& Schmidt, 2017, S. 4).

Das Netz der Netze ist in seiner noch kurzen Geschichte nicht nur zu einem immer erfolgreicheren Kommunikations- und Informationsmedium geworden; es ist zugleich das digitale Herz der globalisierten Welt. [. . . ] Doch nicht nur die Wirtschaft, auch Wissenschaft und Forschung, Politik und Kultur finden längst zu einem nicht geringen Teil im oder über das Internet statt. Tendenz steigend (Münker, 2009a, S. 1).

Eng verwoben mit „Social Media“, begrifflich auch oft synonym gebraucht, aber doch eher als die Konstitutionsbedingung sozialer Medien zu verstehen, ist „Web 2.0“. Für Stefan Münker (2009) ist das mehr als nur ein Schlagwort: 
Der Begriff „Web 2.0“ ist [ . . ] eine Chiffre für eine ebenso radikale wie unaufhaltsame Veränderung nicht nur unserer digitalen Medien, sondern unserer Welt (Münker, 2009b, S. 28).

Ein Bestandteil des „Web 2.0“, ein ebenfalls weit aufgespannter und dabei längst nicht eindeutig definierter Begriff, sind soziale Medien. Diese können selbst auch wieder in diverse Gattungen unterteilt werden. Den Zusammenhang von sozialen Medien und dem Web 2.0 beschreibt Münker so:

Die sozialen Netzwerke sind das virale Zentrum des zentrumslosen Web 2.0 und der derzeit wichtigste Motor seiner Entwicklung (Münker, 2009b, S. 87).

Auf die Bedeutung, welche dem Web 2.0 insbesondere im Zusammenhang mit Wissen zukommt, macht Elfriede Sixt (2014) aufmerksam:

\begin{abstract}
Als grundlegendes Prinzip des Web 2.0 gilt der Mitmachgedanke, der vorher eher passive Internetnutzer bringt sich auf speziellen Plattformen aktiv ein und steuert eigene Inhalte bei (Partizipation, Vernetzung und Austausch). Mit dem Web 2.0 vollzieht sich die Änderung im Umgang mit dem Internet weg vom Abrufmedium hin zum Partizipationsinstrument. Vor allem das Thema Wissen bekommt durch den Mitmachgedanken des Web 2.0 eine völlig neue, nämlich kollektive Dimension verliehen. Web 2.0 erleichtert nicht nur den Zugang und Austausch von Wissen beträchtlich, sondern motiviert auch ungemein, einen eigenen Beitrag zu leisten (Sixt, 2014, S. 8)
\end{abstract}

Zum ersten Mal öffentlich erwähnt wurde der Begriff „Web 2.0“ Ende 2003 in der US-amerikanischen Ausgabe Fast Forward 2010 - The Fate of IT der Zeitschrift CIO in dem Artikel 2004: The Year of Web Services von Eric Knorr (2003), dem Chefredakteur des IDG Magazins InfoWorld:

An increase of outsourcing with web services is nothing less than the start of what Scott Dietzen, CTO of BEA Systems, calls the Web 2.0, where the Web becomes a universal, standards-based integration platform. Web 1.0 (HTTP, TCP/IP and HTML) is the core of enterprise infastructure (Knorr, 2003, S. 90).

Weiter geprägt haben den Begriff Dale Dougherty und Craig Cline, die im Kontext der Vorbereitung einer Konferenz nach einer treffenden Bezeichnung für den damaligen Wandel des Internets von einem Rezipienten- hin zu einem SendeMedium suchten. Im Jahr 2004 fand schließlich die erste Web 2.0 Konferenz in Kalifornien statt; bis ins Jahr 2011 wurde diese jährlich wiederholt. Gefestigt hat sich „Web 2.0“ als Begriff dann mit dem 2005 erschienen Essay What is Web 2.0? Design Patterns and Business Models for the Next Generation of Software von Tim 
O’Reilly (2005). Darin werden das damals neuartige, interaktive Technologieangebot und dessen Anwendungen sowie auch das veränderte Nutzerverhalten der Internetteilnehmer analysiert. O'Reilly definiert Web 2.0 ähnlich wie zuvor Knorr als neue Bewegung in der IT-Industrie hin zum Internet als Plattform (Alpar, \& Blaschke, 2008, S. 4; Bernauer, Hesse, Laick, \& Schmitz, 2011, S. 17 f.; O'Reilly, 2005a; Schmidt, 2018, S. 16; Schmidt, 2008, S. 19 ff.; Sixt, 2014, S. 7; Taddicken, \& Schmidt, 2017, S. 5):

Web 2.0 is the network as platform, spanning all connected devices; Web 2.0 applications are those that make the most of the intrinsic advantages of that platform: delivering software as a continually-updated service that gets better the more people use it, consuming and remixing data from multiple sources, including individual users, while providing their own data and services in a form that allows remixing by others, creating network effects through an "architecture of participation," and going beyond the page metaphor of Web 1.0 to deliver rich user experiences (O'Reilly, 2005b).

Grundsätzlich ist das Suffix ,2.0“ in der Softwareentwicklung gebräuchlich, wo es die erweiterte und optimierte Version eines Programms beschreibt. Demnach wäre das Web 2.0 die neuere und optimierte Variante des Web 1.0. Mit der Begriffswahl hat der Essay von O'Reilly zwar einen fundamentalen Wandel des Internets suggeriert, doch die technologische Weiterentwicklung erfolgte nicht sprunghaft, sondern sukzessive; einen Moment, in dem das Web 1.0 abgeschaltet und durch das Web 2.0 ersetzt worden wäre, hat es nie gegeben. Innovationen in der Softwaretechnik gingen stattdessen einher mit Fortschritten in der Leistungsfähigkeit und auch der Verbreitung des Personal Computer sowie neuen Übertragungstechnologien. Wiederholt hat sich diese Entwicklung zuletzt im Kontext mobiler Technologien, durch Innovationen in der Hardwaretechnik wie Smartphones und Tablets sowie erneut in den Übertragungstechnologien durch UMTS und LTE, die in Verbindung mit dem Fortschritt im Softwarebereich, zum Beispiel in Gestalt von Apps die gegenwärtige Form der Onlinekommunikation bestimmen, die nun auch mobil ist (Bernauer et al. 2011, S. 17; Schmidt, 2018, S. 10, S. 16; Schmidt, 2009, S. 19 ff.; Taddicken, \& Schmidt, 2017, S. 5 ff.). 6

\footnotetext{
${ }^{5}$ Für einen Überblick über die Entwicklung des Internets hin zum Web 2.0, in dem es Nutzern möglich ist, Inhalte zu generieren, die dann ,User-generated Content“ genannt werden, siehe: Goldbeck, 2013, S. 3 f.; Kaplan, \& Haenlein, 2012; Schmidt, 2009, S. 11 ff.; Sudulich et al., 2014, S. 5 ff..

${ }^{6}$ Inzwischen ist bereits die Rede von einem Übergang in das „Web 3.0“ oder auch „Semantic“ Web, wie bei Tuten und Solomon (2018, S. 8 f.).
} 
Zudem sind im Kontext der Diskussion um das Web 2.0 die sozialen Aspekte von seiner Nutzung ins Zentrum der Betrachtung gerückt:

In dieser Hinsicht spricht für die Annahme eines grundlegenden Wandels, dass das Web 2.0 vor allem auch soziale Innovationen mit sich bringt. Soziale Innovationen stellen neue Wege dar, Ziele zu erreichen, die die Richtung des sozialen Wandels verändern und soziale bzw. individuelle Probleme und Herausforderungen besser lösen können [... ]. Das Web 2.0 hat durch seine vereinfachte Bedienbarkeit ohne spezielle technische Fertigkeiten die individuellen Partizipationsmöglichkeiten an (teil-)öffentlichen Diskursen und sozialen Mechanismen gestärkt und zu einer grundlegenden Veränderung des Kommunikations- und Mediennutzungsverhaltens geführt. Technische, ökonomische und soziale Innovationen zusammen lassen es somit gerechtfertigt erscheinen, von einer veränderten Konstellation zu sprechen, die den Unterschied zwischen einem „Web 1.0“ und einem „Web 2.0“ gerechtfertigt scheinen lassen (Taddicken, \& Schmidt, 2017, S. 7).

Ein 2010 unter dem Titel Users of the world, unite! The challenges and opportunities of Social Media erschienener Artikel von Andreas Kaplan und Michael Haenlein (2010) gilt heute als Meilenstein hinsichtlich der Frage nach einer allgemeinen Definition von sozialen Medien sowie einer Konkretisierung ihrer verschiedenen Ausprägungen:

[... ] Social Media is a group of internet-based applications that build on the ideological and technological foundations of Web 2.0, and that allow the creation and exchange of User Generated Content (Kaplan, \& Haenlein, 2010, S. 61). 
Kaplan und Haenlein thematisieren soziale Medien zusammengefasst als einen neuen, revolutionären Trend und diskutieren seine Relevanz für Unternehmen; dabei betonen sie die partizipative und kollaborative Organisation der vernetzten Nutzer. Verschiedene Erscheinungsformen sozialer Medien klassifizieren sie in insgesamt sechs Gruppen: (1) Collaborative Projects, (2) Blogs, (3) Content Communities, (4) Social Networking Sites, (5) Virtual Game Worlds, (6) Virtual Social Worlds (Kaplan, \& Haenlein, 2010; Gabriel, \& Röhrs, 2017, S. 15 f.). ${ }^{7}$

Innerhalb unterschiedlicher Gattungen von Social Media stehen deren Nutzern diverse Technologien zur Verfügung, die verschiedene Funktionen und Dienste ermöglichen. Laura Sudulich, Matthew Wall, Rachel Gibson, Marta Cantijoch und Stephen Ward (2014) beschreiben diese:

[... ] Web 2.0 environment is characterised by the presence of several applications and services that facilitate user generation, sharing, editing and tagging of content, as well as the creation of networks of connected users - such services and applications include blogs and blog-creating software, wikis, multimedia-sharing sites, tagging and social bookmarking sites, podcasting and social media sites. These technologies both emerge from and contribute to a series of 'big ideas' that underpin the Web 2.0 Internet - most notably a focus on facilitating the production and sharing of user-generated content, but also a deep awareness of network effects and the 'power of the crowd', as well as a focus on using large-scale data as a technical and economic resource [ . . . ] (Sudulich et al., 2014, S. 6 f.)

\footnotetext{
${ }^{7}$ Im Rahmen dieser Forschungsarbeit wird, weiter als bereits mit Haenlein und Kaplan geschehen und mit Taddicken und Schmidt (2017) noch folgend, darauf verzichtet, diverse Social-Media-Gattungen gesondert zu thematisieren und durch Typisierungen oder Klassifikationen voneinander abzugrenzen. Zum einen gründet diese Entscheidung in forschungsökonomischen Erwägungen, zum anderen erscheint eine derartige Systematisierung nicht als eine primäre Aufgabe der Soziologie. Es ist jedoch anzumerken, dass eine konsequente und systematische Trennung kaum möglich ist, da verschiedene Gattungen im Hinblick auf Plattformen und Angebote ineinander übergehen und ferner die Innovationsgeschwindigkeit sozialer Medien derart hoch ist, dass eine Systematisierung stets nur vorübergehend sein kann, also über kurz oder lang veraltet und unvollständig erscheint (Taddicken, \& Schmidt, 2017, S. 9). Trotzdem existiert hinreichend Literatur, die den Versuch der Systematisierung von Social Media unternimmt, zum Beispiel: Alpar, \& Blaschke, 2008; Ebersbach, Glaser, \& Heigl, 2011, S. 37 ff.; Gabriel, \& Röhrs, 2017, S. 20 ff.; Kaplan, \& Haenlein, 2010, 2012; McCay-Peet, \& Quan-Haase, 2017, S. 15 ff.; Michelis, \& Schildhauer, 2012; Schmidt, 2018, S. 11 ff.; Schmidt, \& Taddicken, 2017b; Schmidt, 2009, S. 22 ff.; Sixt, 2014, S. 9 ff.; Taddicken, \& Schmidt, 2017, S. 9 ff..
} 
Von Seiten der Kommunikationswissenschaft, hier vertreten durch Monika Taddicken und Jan-Hinrik Schmidt (2017), werden als Gattungen sozialer Medien benannt: (1) Soziale Netzwerkplattformen, wie das schon erwähnte Facebook oder auch Google + und für den beruflichen Kontext Xing und LinkedIn, (2) Multimediaplattformen für den Austausch von Fotos und Videos, beispielsweise das bereits angesprochene YouTube, zudem Instagram oder Snapchat, (3) eine Vielzahl an Weblogs, die meist nur „Blogs“ genannt werden, sowie deren Sonderform Microblogs, insbesondere in Gestalt von Twitter, das schon genannt wurde, (4) Instant-Messaging-Dienste wie WhatsApp und (5) Wikis, in erster Linie in Gestalt von Wikipedia (Taddicken, \& Schmidt, 2017, S. 4 f., S. 9 ff.; Schmidt, 2018, S. 11 ff.). Einer sozialwissenschaftlichen Perspektive folgend begreifen Taddicken und Schmidt „Social Media“ allgemein als einen

[. . . ] Sammelbegriff für Angebote auf Grundlage digital vernetzter Technologien, die es Menschen ermöglichen, Informationen aller Art zugänglich zu machen und davon ausgehend soziale Beziehungen zu knüpfen und/oder zu pflegen (Taddicken, \& Schmidt, 2017, S. 8, Hervorheb. i. O.).

Des Weiteren merken sie an, dass aus sozialwissenschaftlicher Sicht nicht nur Social Media, sondern alle Medien einen sozialen Charakter haben, da sie ein Bestanteil von Kommunikationsakten und Interaktionen oder auch von sozialem Handeln sind. Dieser Umstand klinge mitunter an in Titeln wie „Mitmachweb“, „Participatory Web“ oder „User-generated Content“, einem weiteren Schlagwort im Kontext von Web 2.0 und Social Media. ${ }^{8}$ Allen sozialen Medien sind gemäß Taddicken und Schmidt neben gegebenenfalls noch weiteren Zielen mindestens die des kommunikativen Austausches und der sozialen Interaktion gemein (Taddicken, \& Schmidt, 2017, S. 4 f.):

\footnotetext{
${ }^{8}$ Die Genese von User-generated Content (nutzergenerierte Inhalte) wird auch unter dem Stichwort „Produsage“ thematisiert. Diese Begriffswahl betont, dass aktive Nutzer sozialer Medien (im Englischen ,user“) deren Inhalte nicht nur rezipieren, sondern auch produzieren (im Englischen „,to porduce“) und daher als „Produser“ anzusehen sind. Siehe zu Produsage beispielsweise: Schmidt, 2008, S. 25 ff.. Für eine Definition des Begriffs „User-generated Content" siehe: Sixt, 2014, S. 15.
} 
Soziale Medien verändern damit auch die Strukturen gesellschaftlicher Öffentlichkeit: Sie brechen die Dominanz des Modus „Massenkommunikation“ und seiner institutionalisierten Formen, indem sie andere Modi, Arenen und Kontexte bereitstellen, die Kommunikationsinhalte für einen (potenziell) großen Personenkreis sichtbar und zugänglich machen [... ]. Informationen in sozialen Medien sind persistent, replizierbar sowie - aufgrund ihrer Digitalität - auch durchsuchbar [. . .], und aggregierbar [. . . ]. Es besteht eine hohe Transparenz von Daten, Vernetzungen und Handlungen (Taddicken, \& Schmidt, 2017, S. 5).

Gegenüber einer sozialwissenschaftlichen Betrachtung sozialer Medien fokussiert die Wirtschaftsinformatik, auch wenn sie bemüht ist, ,den Mensch in den Vordergrund“ zu stellen, in ihrer Auseinandersetzung mit Social Media technologische Aspekte. Roland Gabriel und Heinz-Peter Röhrs (2017) schlagen folgende Definition vor:

Social Media (soziale Medien) sind digitale Medien, Technologien und Systeme, die über Anwendungsplattformen angeboten werden und mit denen unterschiedliche Anwendungen (Social-Media-Anwendungen) im Internet (bzw. im Intranet) über Web 2.0 oder Web 3.0 ausgeführt werden können. Hierzu können sich z. B. Social Networks (soziale Netzwerke) als ein gemeinschaftliches Netzwerk von Benutzern bilden, die die Social-Media-Technologien nutzen. Charakteristisch für die Anwendungen sind die Kommunikation und vor allem die Erstellung (user-generated content) und die Verteilung von Inhalten, die in digitaler Form bzw. als digitale Medien vorliegen (Gabriel, \& Röhrs, 2017, S. 16, Hervorheb. i. O.).

Die Sozial- und Geisteswissenschaften hingegen bestimmen soziale Medien gemeinsam mit der Informatik, etwa Lori McCay-Peet und Anabel Quan-Haase (2017), so:

Social media are web-based services that allow individuals, communities, and organizations to collaborate, connect, interact, and build community by enabling them to create, co-create, modifies [sic], share, and engage with user-generated content that is easily accessible (McCay-Peet, \& Quan-Haase, 2017, S. 17).

Ungeachtet dessen, welche Definition von Social Media zugrundgelegt wird, ist klar festzustellen, dass sich soziale Medien in den letzten Jahren in großer Geschwindigkeit verbreitet haben; nicht nur in Deutschland, sondern auf der ganzen Welt. 
In Deutschland wird diese Entwicklung von mehreren, regelmäßig durchgeführten Studien begleitet: die Berichte der Arbeitsgemeinschaft Online-Forschung (AGOF), die ARD/ZDF-Onlinestudien, die Allensbacher Computer- und TechnikAnalysen (ACTA), die Untersuchungen des Bundesverbands Informationswirtschaft, Telekommunikation und neue Medien (BITKOM). Gemäß diesen Studien liegt die Internetnutzung der deutschen Bevölkerung bei $90 \%$ (ARD/ZDFOnlinestudie) beziehungsweise bei $85 \%$ (AGOF). ${ }^{9}$ Alle Studien verweisen darauf, dass Social Media in den letzten Jahren in ihrer Verbreitung wie Nutzung erheblich zugenommen haben; derzeit ist allerdings eine Stagnation zu verzeichnen. So wird beispielsweise Facebook von $33 \%$ der Deutschen mindestens einmal pro Woche genutzt; Instagram liegt hier bei $9 \%$, Videoportale wie YouTube erreichen $31 \%$, Twitter erzielt $3 \%$ und WhatsApp hat sich auf $64 \%$ gesteigert (ARD/ZDF-Onlinestudie). Ferner geben die Studien in Bezug auf die Nutzungsmotive und -zwecke ein sehr ähnliches Ergebnis wieder; sie bestehen vorrangig in der Pflege sozialer Kontakte. Seit einigen Jahren kommt Videoformaten eine gestiegene Relevanz zu; aktuell ist jedoch auch hier eine Stagnation festzustellen. Auch die Nutzung von Fanseiten in Netzwerkplattformen, zum Beispiel von Automobilmarken, weist ein hohes Niveau auf. Interessant sind aber vor allem auch die Erkenntnisse, die ein Blick auf die Nicht-Nutzer sozialer Medien eröffnet: Etwa ein Viertel derjenigen Deutschen, die online sind, nutzt Wikipedia nicht, circa ein Drittel dieser Gruppe verwendet keine Video-Portale und ungefähr die Hälfte gehört keiner sozialen Netzwerkplattform an. Die im Vergleich hohen Anteile der Nicht-Nutzer von Social Media erstrecken sich dabei in erster Linie, allerdings nicht ausschließlich auf ältere Jahrgänge. Jedoch ist ebenso ein Bevölkerungsanteil auszumachen, der persönlich weder Sinn noch Nutzen in sozialen Medien erkennt; die Sorge um einen potentiellen Datenmissbrauch und die Wahrung der Privatsphäre gelten als gewichtige Gründe. Im Rahmen einer Betrachtung der Nicht-Nutzer von Social Media fällt ferner auf, dass nahezu ein Viertel der Deutschen offline ist; diskutiert wird dieser Umstand unter den Ausdrücken „digitale Spaltung“ und „Digital Divide“. Damit ist angesprochen, dass soziale Gleichheit und Gerechtigkeit in Gefahr sind, wenn bestimmte soziale Gruppen von der Internetnutzung profitieren, andere hingegen offline sind. Der Blick auf derartige Ungleichheiten hat sich allerdings mittlerweile vom reinen Zugang zum Internet auf den Umgang damit sowie auch in Richtung der Fähigkeiten, die dazu vonnöten sind, verlagert; neben einer Alters- sind eine Geschlechter- sowie eine

\footnotetext{
${ }^{9}$ Der Unterschied beruht auf der unterschiedlichen Grundgesamtheit, die den beiden Studien zugrunde liegt. Die ARD/ZDF-Onlinestudie bezieht sich auf die deutschsprachige Bevölkerung ab 14 Jahren, die Untersuchung der AGOF auf die deutschsprachige Bevölkerung ab 16 Jahren.
} 
Bildungskluft festzustellen (ACTA, 2016; AGOF, 2018, 2017, 2015; BITKOM, 2013; Koch, \& Frees, 2017; McCay-Peet, \& Quan-Haase, 2017, S. 14; Projektgruppe ARD/ZDF-Multimedia, 2017; Schneller, 2013; Sudulich et al., 2014, S. 3, S. 10; Taddicken, \& Schmidt, 2017, S. 13 ff.):

Hence, it is important to realize that social media adoption, usage, and its social implications are dynamic social processes that occur within existing patterns of inequality: some social groups are simply being left out of the social media conversation (McCay-Peet, \& Quan-Haase, 2017, S. 14).

In Deutschland scheint heute, wie auch in Europa und den USA, die Nutzung sozialer Medien fester Bestandteil der Interaktion und Kommunikation eines Großteils der Bevölkerung zu sein; soziale Medien gelten dort als gesellschaftlich etabliert. Weltweit sind aber nach wie vor erhebliche Ungleichheiten auszumachen. Auch die Unterschiede zwischen Digital Natives und Digital Immigrants dürfen nicht übersehen werden. Hierbei handelt es sich um zwei Bezeichnungen, die darauf aufmerksam machen, dass die heutige Gesellschaft zum einen aus Individuen besteht, die mit den Entwicklungen des Internets aufgewachsen sind oder in diese hineingeboren wurden und sich eine Offline-Welt kaum noch oder gar nicht mehr vorstellen können, als auch aus solchen, die, vor allem aufgrund ihres Geburtsjahres, eben dieser Offline-Welt entstammen (Günther, 2007; McCay-Peet, \& Quan-Haase, 2017, S. 14; Sudulich et al. 2014, S. 3; Taddicken, \& Schmidt, 2017, S. 13 ff.). ${ }^{10} 11$

Soziale Medien bedeuten auch für Organisationen, wie Unternehmen sie darstellen, und deren Praxis eine facettenreiche Transformation. Rhagavendra Rao (2017) in dem von ihm herausgegebenen Sammelband Social Media Listening und Monitoring for Business Applications dar, wie soziale Medien Unternehmen weltweit verändern. Vor allem die für F\&E, Vertrieb, Marketing und Kommunikation zuständigen Unternehmensbereiche würden sich Social Media zu Nutze machen. Erfolgreiche Anwendungsfälle fänden sich in Produktentwicklung und Kundendienst:

${ }^{10}$ Angaben zur Nutzung von Internet und sozialen Medien hält in Bezug auf Europa das Statistische Amt der Europäischen Union (Eurostat) bereit (https://ec.europa.eu/eurostat/de/home); in Bezug auf die USA bieten sich die vom Pew Research Center durchgeführten Studien des Pew Research Internet Project und für weltweite Informationen die Internetseite Internet World Stats (https://www.internetworldstats.com/) sowie auch der Web Index der World Wide Web Foundation (http://thewebindex.org/) an (Taddicken, \& Schmidt, 2017, S. 17 f.).

${ }^{11}$ China ist für die deutsche Automobilindustrie ein zentraler Absatzmarkt und weist eine eigene Social- Media-Landschaft auf. Innerhalb der vorliegenden Arbeit wird diese aus forschungsökonomischen und -strategischen Gründen nicht näher betrachtet. Siehe hierzu: He, 2017; Liu, 2016; Xie, 2017. 
Further social media is changing business landscape across the borders of countries. [... ] Social media tools empower all the stake holders of business enterprise to integrate most of the functional areas in the business enterprises for business processes and decisions. Mainly research, marketing, sales customer services and product development functions in organizations can be included within the social media programs (Rao, 2017, S. XVI).

Ebenso erkennen Rajagopalan Venkatesh und Sudarsan Jayasingh (2017) eine Transformation der Wirtschaft aufgrund von Social Media, deren Bedeutung sie für Organisationen und auch für deren Kunden sehen. Vor- wie Nachteile, die soziale Medien für Unternehmen bereithalten, werden diskutiert:

\begin{abstract}
Although Social networks can help companies spread good news fast, it can also spread bad news just as quickly. Moreover, if customers want to vent their anger on your product or service, they can use your social network account. Managers need to understand how to handle those situations quickly and effectively. Also, as social media is not as widely moderated or censored as mainstream media, individuals can say anything they want about a company or brand, positive or negative. Today's customers act, and react, at lightning speed to your latest products, pricing plans and sales channels. They spread the word about their good and bad experiences with comments captured across a dizzying range of platforms. [. . . ] But marketers need to quickly and cost-effectively collect social media data from various sources, analyze the data, and understand the results before sudden market changes undermine sales or damage their brands. This rapid response requires the discovery, extraction, cleansing and integration of a wider range of data than ever before. When rumors on social media threaten a product's reputation, or a complaint about an out-of-stock product signals an inventory crisis, marketers and brand managers need detailed information immediately. They must be able to analyze and correlate many more types of customer data, sense danger and opportunity in their customers' experiences much more quickly, and response much more effectively than ever before. It also requires social media tracking and analytic tools, natural-language processing to draw insights from text, advanced predictive analytics to aid decisionmaking and easy-to-use dashboards and reports (Venkatesh, \& Jayasingh, 2017, S. 9).
\end{abstract}

Basierend auf dieser Erkenntnis benennen Venkatesh und Jayasingh Möglichkeiten für den Einsatz sozialer Medien in bestimmten Unternehmensbereichen oder Funktionen: E-Commerce (Electronic Commerce), Marketing, Communication, CRM, Human Resource Management (HRM), Knowledge Management. Um soziale Medien im Unternehmenskontext erfolgreich „zu managen“, empfehlen sie, ein „Social Media Command Center" einzurichten; in einem solchen sollten sämtliche mit sozialen Medien zusammenhängenden Aktivitäten unternehmensweit koordiniert werden (Venkatesh, \& Jayasingh, 2017). 
Im Rahmen einer umfangreichen Analyse hat Kijpokin Kasemsap (2017) die Literatur zu Social Media mit Fokus auf die moderne Wirtschaftswelt ausgewertet und anhand der Ergebnisse eine regelrechte Lobeshymne auf soziale Medien gesungen. Potentiale erkennt Kasemsap vor allem für: Marketing, Communication Management, Knowledge Sharing. Er kommt zu folgendem Fazit:

Social media plays a growing role in business communication and becomes important platforms for marketing and advertising. Social media offers a chance to redefine the delivery of service to customers, thus changing the way they think about a company's brands while considerably lowering service costs. Social media has many benefits, such as the increased awareness of the organization, the increased traffic to website, the greater favorable perceptions of the brand, the better understanding of customer perceptions of their brand, the improved insights into target markets, and the improved identification of new product opportunities in modern business. Social media allows organizations to improve communication and productivity by disseminating information among the different groups of employees in a more efficient manner. The most obvious opportunity of utilizing social media is to generate business revenue through building a business community and advertising products within social media platforms. When considering social media campaigns, social media users can try to attract followers with promotions. Social media is an important approach to attracting new customers, enhancing brand management, and promoting knowledge management in modern organizations. Using social media allows customers to connect and interact with business on a more personal level. Applying social media has the potential to enhance organizational performance and reach strategic goals in modern business (Kasemsap, 2017, S. 28).

Neus Soler-Labajos und Ana Jiménez-Zarco (2017) wiederum beschäftigen sich mit der Rentabilität im Social Web. Ausgehend von dem Schlagwort „Enterprise 2.0" setzen sie sich mit dem Einsatz sozialer Medien in Unternehmen auseinander und sehen Vorteile dadurch in Bezug auf die folgenden drei Aspekte: (1) Image and Brand Reputation, (2) Communication, (3) Traffic to the Corporate Website. Soler-Labajos und Jiménez-Zarco erklären Verbesserungen letztlich durch den Einsatz von Social-Media-Analysen, weisen aber ebenso darauf hin, dass das Web 2.0 als soziologisches Phänomen einen bedeutsamen kulturellen Wandel markiert: 
Listening, talking, sharing, and generating content that interests the user is the premise of this new form of interactive relationship between the company and the public. Web 2.0 is the environment in which companies meet the opinions of brand users. Social media provide the most effective tools to meet customers, receive feedback, and disseminate information in an effective and efficient way. Social networks represent communities, much like ecosystems, of coexistence between people and brands, where the latter are 'humanized' while maintaining their business objectives. [. . . ] To convert information into knowledge and guide decision making, we use methods of measurement to determine whether objectives have been achieved and what value social media are contributing to the economic health of the company. To develop these measurements, we must choose optimal metrics, both quantitative and qualitative, that reflect the company context. Web 2.0 is a sociological phenomenon that represents an important cultural change. Companies must not only adapt to this change but also pay attention to a new series of concepts and techniques that will help them advance even more, by making them mobile and able to predict the market using semantic web technologies (Soler-Labajos, \& Jiménez-Zarco, 2017, S. 63 f.).

Trends, Chancen und Risiken, die soziale Medien für Unternehmen mit sich bringen, beleuchten auch Gabriel und Röhrs, deren Definition sozialer Medien bereits aufgeführt wurde. Folgende Chancen erkennen sie: Durchführung von Marktforschung; Steigerung des Bekanntheitsgrads; Kontakt zu und Dialog mit Kunden; positive Reputation; Publikation von Fachinformationen; virale Marketing-Effekte; Integration von Kunden in die Produktentwicklung; Lösung komplexer Probleme über Crowd Sourcing; Förderung von E-Commerce; Erreichen jüngerer Zielgruppen; Stärkung der Kundenbindung; Kooperation mit Multiplikatoren; Kanal zu Menschen; Umsatz- und Gewinnsteigerung. Neben diesen Potentialen sehen Gabriel und Röhrs jedoch ebenso einige Risiken für Unternehmen: Datenschutzprobleme; Wissensdefizite; ausbleibende Reaktion der Zielgruppe; fehlerhafte Umsetzung mangels Konzept und schlechte Implementierung; Kontrollverlust; ausbleibende interne Kooperation; unzureichende Ressourcen; mangelhafte Organisation und ungeklärte Zuständigkeit; fehlende Affinität der Zielgruppe; interne Widerstände; negative Publicity; Einbußen bei Umsatz und Gewinn; Zeitverschwendung aufgrund des nicht vorhandenen Fokus; Angst vor dem virtuellen Pranger; Suchtpotential; Kriminalität; schwierige Messbarkeit von Erfolgen; negative Reputation; Gefahr von Fehlinformationen; Gehaltlosigkeit der Inhalte. Insgesamt wird Social Media eine enorme Bedeutung für Unternehmen zugemessen und deren Zukunft daher anhand von fünf Thesen prognostiziert: (1) Soziale Medien etablieren sich als Querschnittsfunktion und zentraler Aufgabenbereich; (2) soziale Medien halten Einzug in die Produktentwicklung, vor allem hinsichtlich Konfiguration und Design; (3) soziale Medien beeinflussen Werbekampagnen und Maßnahmen der Public Relations (PR) entscheidend; (4) 
soziale Medien modifizieren und optimieren CRM-Prozesse und E-CommerceAktivitäten; (5) soziale Medien bedingen, vor allem in Bezug auf interne wie externe Kommunikation, neue Unternehmensstrategien. Somit halten Gabriel und Röhrs zusammenfassend fest:

Der erfolgreiche Einsatz von Social Media in Unternehmen hängt schließlich von einer fundierten betrieblichen Social-Media-Strategie und von einer fachlichen Qualifikation der Mitarbeiter ab. Sowohl die Chancen als auch die Risiken von Social Media müssen erkannt und analysiert werden, vor allem bei Beachtung der Wirtschaftlichkeit, d. h. der Kosten und des Nutzens, und der Qualität der Social-Media-Anwendungen (Gabriel, \& Röhrs, 2017, S. 88, Hervorheb. i. O.).

Zuletzt sei noch einmal von der gesellschaftlichen Mesoebene und der Thematisierung der Relevanz sozialer Medien für Organisationen auf die Makroebene zurückgekehrt. Münker fasst die sozialen Folgen, die das Web 2.0 und dessen Ausprägungen bedingen, wie folgt zusammen:

Das Web 2.0 hat nicht nur das Internet radikal verändert - es hat die digitale Medienrevolution, die einzig vergleichbar ist mit den großen Umwälzungen durch die Erfindung des Buchdrucks oder der Elektrifizierung, zu ihrem gegenwärtigen Höhepunkt geführt. Von hier können wir gegenwärtig überall im Internet die Emergenz digitaler Öffentlichkeiten beobachten - als Effekte jener spezifischen Praktiken des Umgangs mit Informationen, der Herstellung kultureller Güter und der Verbreitung von Wissen und Meinungen, die erst und nur die vernetzten Plattformen digitaler Medien ermöglichen konnten. Die Entstehung einer radikal anderen, utopischen Gegenwelt, wie sie die Netzidealisten der ersten Stunde erträumt hatten, sehen wir hier nirgendwo; wir sehen aber, wie die digitalen Öffentlichkeiten heute bereits über das Netz hinaus zu wirken beginnen - in die Politik, die Wissenschaft, in Wirtschaft, Kunst und den Journalismus; und natürlich in unsere alltäglichen sozialen Beziehungen. Das ist kein Zufall - unsere Mediengesellschaft ist zugleich eine Netzwerkgesellschaft und lebt von vernetzten Medien. Und so treffen wir, hegelianisch gesprochen, in den Sozialen Netzmedien des Web 2.0 schlicht auf die zeitgemäße Erscheinung von Medien, die implizit immer schon sozial sind. Dabei steht der strukturelle Wandel, den die Entstehung digitaler Öffentlichkeiten als Effekt der medialen Vernetzung für unsere Gesellschaft bedeutet, erst am Anfang. Der Ausgang mag offen sein, die Richtung ist es nicht (Münker, 2009b, S. 132 f.). 
In dem Bewusstsein der Heterogenität und Unbestimmbarkeit sozialer Medien, welche schlicht in deren rasanter Weiterentwicklung begründet liegt, galt es trotzdem eine für den weiteren Verlauf dieser Arbeit, insbesondere für deren empirischen Teil geltende Definition von Social Media festzulegen. Diese sollte klar und einfach formuliert sein. Die Wahl ist auf eine 2013, im Rahmen des 25. Plenums der Fachgruppe NEON des Berufsverbands Deutscher Markt- und Sozialforscher entwickelte Definition gefallen:

Social Media ist eine Vielfalt digitaler Medien und Technologien, die es Nutzern ermöglicht, sich auszutauschen und mediale Inhalte einzeln oder in Gemeinschaft zu gestalten. Die Interaktion umfasst den gegenseitigen Austausch von Informationen, Meinungen, Eindrücken und Erfahrungen sowie das Mitwirken an der Erstellung von Inhalten. Die Nutzer nehmen durch Kommentare, Bewertungen und Empfehlungen aktiv auf die Inhalte Bezug und bauen auf diese Weise eine soziale Beziehung untereinander auf. Die Grenze zwischen Produzent und Konsument verschwimmt . . . Als Kommunikationsmittel setzt Social Media einzeln oder in Kombination auf Text, Bild, Audio oder Video und kann plattformunabhängig stattfinden (Scheffler, 2014, S. 13).

Um die weite Landschaft sozialer Medien bildlich zu veranschaulichen, gruppiert das Unternehmen ethority global network GmbH in seinem Social-Media-Prisma zentrale Anbieter sozialer Medien. Das Prisma liegt für die deutsche wie globale Perspektive in einer aktualisierten Version von 2017 vor; die chinesische Landschaft sozialer Medien wurde zuletzt 2012 abgebildet. Über 250 Anbieter sind in 25 Kategorien aufgelistet; dabei umfassen die Dienste der Anbieter unter anderem die Bereiche Text, Bild, Audio, Video, Musik, Social Gaming, Bookmarking (Lütters, \& Egger, 2013, S. 34; Sten, 2017a, 2017b, 2012). Abbildung 3.1 zeigt das momentan aktuelle Social-Media-Prisma zu Deutschland.

Eine Antwort auf die eingangs aufgeworfene und für diese Forschungsarbeit als zu klärend essentielle Frage, was soziale Medien denn überhaupt sind, schlagen Sudulich et al. vor. Mit politikwissenschaftlichem Fokus werden noch einmal der technologische Zusammenhang von Internet, Web 2.0 und Social Media auf den Punkt gebracht und ferner die methodologischen Herausforderungen und Potentiale, die Social Media für die Sozialforschung der Zukunft bergen, betont: 


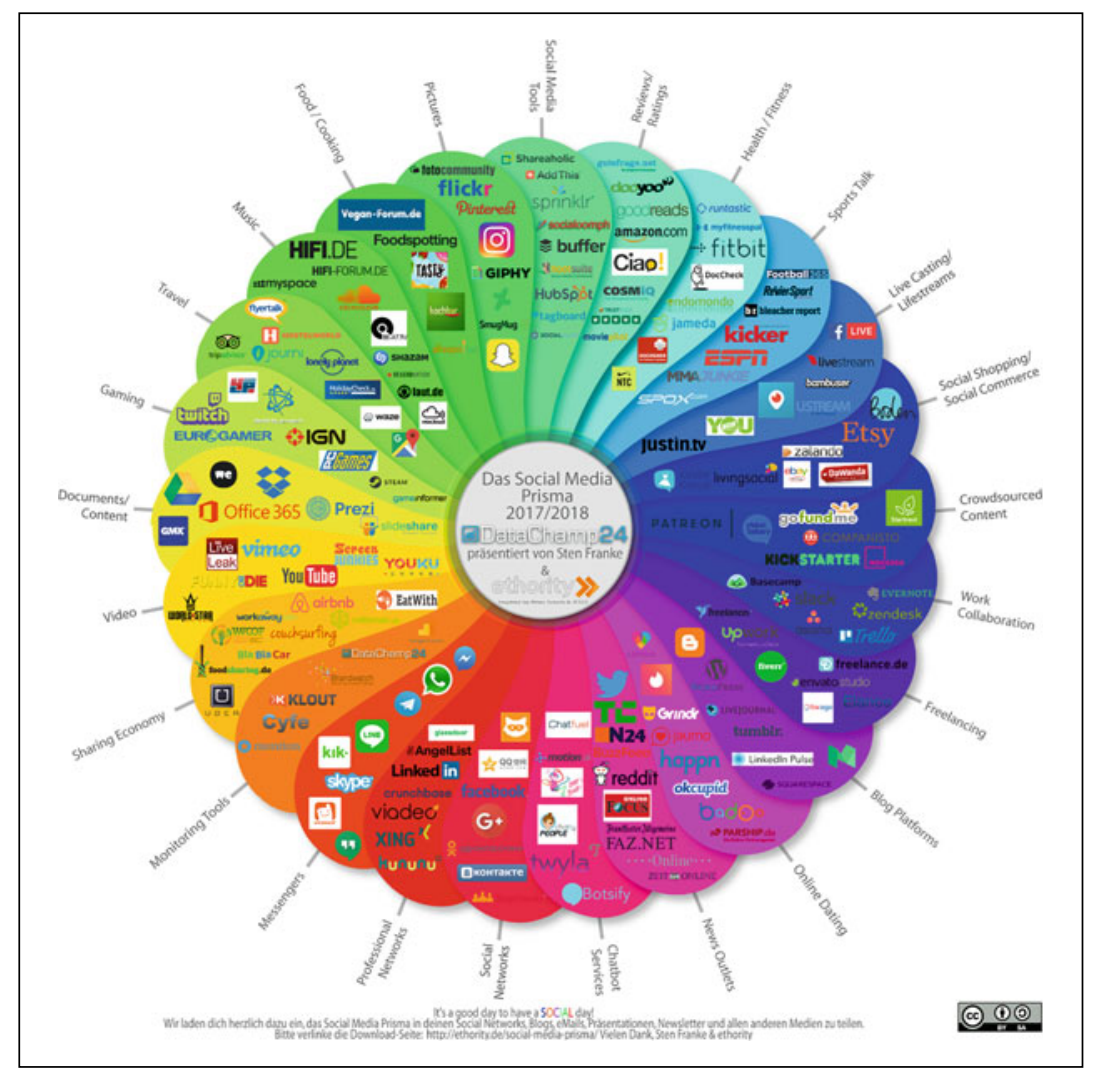

Abbildung 3.1 Social-Media-Prisma 2017/2018, Deutschland (Sten, 2017a) 
A key take away [... ] is that the Internet, the web and social media, while clearly interrelated and interdependent, are not one and the same thing. They form a chronological and technological line of progression whereby the Internet forms the underlying infrastructure and 'root' network that supports the outward 'skein' of the Web, upon which are laid various applications, some of the most popular and widely used of which are social media platforms. From a sociopolitical perspective, they can be seen as an increasingly democratised means of undertaking digital communication, with social media now forming the most widely used entry point into the online world for most people. [. . . ] With rapid advent of Web 2.0 from the mid-2000s onwards, and talk (among some) of a Web 3.0 era emerging [. . . ], we may be in a period of semi-continuous information revolution, which has implications for methodologically rigorous study [... ], especially in the domain of reliability. While this process is fascinating from a theoretical and normative perspective as noted earlier, our concern here is with how, in practical terms, we can make sense of the growing chaos of bits and bytes that it has released into the world of data analysis. The rise of social media in particular presents a new frontier for social science research methodology to confront. [. . . ] [W]e argue that, in its current form, the Internet presents new opportunities for the study of politics and society, providing digitised and instantaneous data on processes and phenomena that were previously extremely difficult to study empirically. However, this medium also poses novel challenges for researchers seeking to abide by the standards of transparency, generalisability and replicability that underlie the 'scientific' claims of empirically orientated social and political research. [. . . ] [A] combination of volume, heterogeneity and plasticity makes the contemporary political Internet a unique research target for social scientists, necessitating considerable methodological care (Sudulich et al., 2014, S. 7 f.). ${ }^{12}$

\footnotetext{
12 Aufgrund des Mangels einer allgemeingültigen Definition von Social Media haben sich mit der Frage, was soziale Medien sind, von Seiten der Kommunikationswissenschaft zudem Carr und Hayes (2015) auseinandergesetzt. Sie haben versucht, eine Definition für Social Media aufzustellen, welche das Potential besitzt bis in 2035 gültig zu sein; diese lautet wie folgt: „Social media are Internet-based channels that allow users to opportunistically interact and selectively self-present, either in real-time or asynchronously, with both broad and narrow audiences who derive value from user-generated content and the perception of interaction with others" (Carr, \& Hayes, 2015, S. 50).
} 


\subsection{Computational Social Sciences oder Digital Humanities}

Online-Forschung erfolgt meist interdisziplinär. Sozialwissenschaften und Informatik können Big Data oder Social Media kaum ohne einander untersuchen; beide Disziplinen sind methodisch aufeinander angewiesen. Diskutiert wird der Zusammenschluss der Sozial- beziehungsweise Geisteswissenschaften mit der Informatik als Computational Social Sciences oder Digital Humanities. ${ }^{13}$

Markus Strohmaier und Maria Zens (2014) setzen sich mit der Analyse sozialer Medien an der Schnittstelle von Informatik und Sozialwissenschaften auseinander und werfen die Frage auf, ob das Zusammentreffen von großen Datenmengen beziehungsweise Big Data mit der Veränderungsgeschwindigkeit, die sie „Internet Time" nennen, eine Krise der Sozialwissenschaften aufgrund methodologischer Herausforderungen bedeutet. Für die Sozialwissenschaften seien computergestützte Verfahren und Prozesse unabdingbar, um „Internet-Transaktionsdaten“ nach sozialwissenschaftlichen Standards extrahieren und analysieren zu können; dies sei Aufgabe der Computational Social Sciences. Bei dieser Kooperation müssen die Sozialwissenschaften laut Strohmaier und Zens ihre Fragestellungen auf die Inhalte des Web 2.0 beziehungsweise die darin stattfindenden Interaktionen

\footnotetext{
${ }^{13}$ Obwohl Big Data nicht das Schwerpunktthema dieser Forschungsarbeit ist, sondern nur im engen Zusammenhang mit sozialen Medien steht, zeigt sich im weiteren Verlauf dieses Abschnitts, was die Sozialwissenschaften darunter verstehen; dies geschieht allerdings eher implizit, denn Big Data wird nicht explizit als ein eigenes Phänomen vorgestellt. In dieser Arbeit ist es nur in seinem Zusammenhang mit Social Media von Interesse. Es ist vor allem die Informatik, die sich als wissenschaftliche Disziplin mit dem Phänomen „Big Data“ beschäftigt und dazu publiziert. Ein Praxishandbuch Big Data. Wirtschaft - Recht - Technik hat Dorschel (2015) vorgelegt. Darin wird darauf hingewiesen, dass Big Data ein mehrdimensionales Phänomen und ein Kunstbegriff zugleich sei; diesen gelte es in heterogene, fachliche Einzelbetrachtungen zu zerlegen und den technischen, kommerziellen, rechtlichen und sozialen Kontext zu beachten. Da Big Data viele unterschiedliche Aspekte umfasse, werde es je nach Zielsetzung und Fragestellung anders definiert und abgegrenzt. Am gängigsten sei in der Informatik eine Definition von Big Data anhand von drei oder vier spezifischen Eigenschaften, die auch die drei oder vier „V's“ genannt werden: Volume, Velocity, Variety, Veracity (Dorschel, \& Dorschel, 2015, S. 1 ff.). König, Schröder und Wiegand (2018) haben demgegenüber einen sozialwissenschaftlichen Sammelband herausgegeben, der Chancen, Risiken und Entwicklungstendenzen von Big Data behandelt und in dem Wachter (2018) Big-DataAnwendungen in der Marktforschung diskutiert. Aus einer techniksoziologischen Perspektive beleuchtet Tufekci (2014) große methodologische Fragen bezüglich Social Media Big Data, etwa die nach Repräsentativität und Validität. Zeller (2014) gibt einen Überblick zu Big Data innerhalb der sozialwissenschaftlichen Online-Forschung.
} 
anwenden; Aufgabe der Informatik sei es, entsprechende Informationen oder „Interaktionsspuren“" zu extrahieren. Jedoch bemerken Strohmaier und Zens, dass die Frage der Datenqualität bisher nicht gelöst wurde, obwohl diese grundlegend ist. Online-Forschung sei, verglichen mit den klassischen Methoden der Sozialforschung, weniger kontrolliert und transparent:

Wir haben darauf hingewiesen, dass das Web zum einen als internationaler Handlungsraum zu sehen ist, zum anderen als ein Raum, in dem Suchmaschinen-Algorithmen und automatisierte Prozesse starken Einfluss haben, manipulative Macht entfalten und Eigendynamiken besitzen, für die Kontrollmechanismen noch nicht entwickelt sind (Strohmaier, \& Zens, 2014, S. 90).

Potential der Computational Social Sciences sehen Strohmaier und Zens also durchaus, jedoch lassen sie die Möglichkeiten zur ökonomischen Einflussnahme und politischen Manipulation, die sich für Organisationen mit sozialen Medien auftun, ebenfalls nicht außer Acht. Soziale Medien begreifen sie auch als einen soziopolitischen Raum:

Organisation und kommunizierte Inhalte der Internet-Medien haben Auswirkungen in der Gestaltung politischer und sozialer Wirklichkeit. Suchmaschinen sind die bevorzugten Instanzen der Informationsbeschaffung, -selektion und -sortierung. [. . . ] Wie wir im Informationsüberfluss navigieren, welche Informationsfragmente wir in welcher Reihenfolge zur Kenntnis nehmen und wie wir die digitale Konstruktion von Wirklichkeit wahrnehmen, wird wesentlich von den Algorithmen der Suchmaschine bestimmt. [. . . ] Die aufklärerische Vorstellung, dass Auswahl und Rezeption neuen Wissens dem gebildeten, bereits informierten, verantwortungsvollen und souveränen Individuum obliegt, war zwar immer eine Idealvorstellung, angesichts dieser technisch unterstützten und manipulierbaren Komplexitätsreduktion mutet sie heute jedoch fast rührend an (Strohmaier, \& Zens, 2014, S. 83 f.).

Computational Social Sciences dürfen also, sofern sie für sich beanspruchen methodisch reflektiert zu sein, nach Strohmaier und Zens die „Macht der Suchmaschinen" nicht aus den Augen verlieren, sondern sollten sich vielmehr stets einen kritischen Blick auf diesen gesellschaftstheoretischen Aspekt bewahren. Durch soziale Medien entstehe eine neue Art sowie auch eine neue Qualität von Daten. Für die Sozialwissenschaften seien neue Methoden zu deren Erhebung, Bereinigung und Evaluation unabdingbar; jedoch gelte es die verschiedenen Einschränkungen und Grenzen von Social-Media-Analysen in die methodologischen Überlegungen einzubeziehen. 
So sehr die technologieinduzierte Datenproliferation die klassische Sozialforschung vielleicht herausfordert, sie bedeutet vor allem eine Chance für die sozialwissenschaftliche Forschung, der - ausgestattet mit den richtigen Fragestellungen und neuen informationellen Verfahren der Datenextraktion - für die Prüfung ihrer Hypothesen zunehmend vollständigere Daten zur Verfügung stehen. Umgekehrt braucht die Computerwissenschaft solche Fragen, um Daten aus computergestützter und getriebener Kommunikation auf soziale Zusammenhänge beziehen zu können. Aus dem für sich genommen nichtssagenden Überfluss Signifikantes zu schöpfen und mithilfe von Big Data komplexe soziale Systeme besser analysieren zu können, ist deshalb ein interdisziplinäres Projekt. Damit aus einer vielversprechenden eine ergebnisreiche Konstellation werden kann, sind weitere Arbeiten notwendig. [. . . ] Dieser methodologischen Herausforderung stellt sich die sich neu formierende Disziplin der Computational Social Science, die die jeweiligen Fachexpertisen - zu Technologien, Methoden, Gesellschaft - zusammenführen möchte [. . . ]. Die Computerwissenschaft leistet den Umgang mit großen Datenbeständen, die mit den statistischen Verfahren der Sozialwissenschaften nicht zu bewältigen sind, liefert Algorithmen und Verfahren des data mining. Die Sozialwissenschaften tragen ihr Wissen über Theorien, Methoden, Datenerhebung, soziales Handeln bei und leisten die Formulierung relevanter Fragestellungen (Strohmaier, \& Zens, 2014, S. 90 f., Hervorheb. i. O.).

Unter den Titeln „Computational Social Sciences“ und „Digital Humanities“ behandelt auch Rob Kitchin (2017) die Kooperation von Sozialwissenschaften und Informatik. Er betrachtet zwar nicht explizit Social Media, sondern eher Big Data, worunter er soziale Medien subsumiert, tut dies aber aus einer sozialwissenschaftlichen Perspektive und setzt somit gewissermaßen die Überlegungen von Strohmaier und Zens fort:

From 2008 onward the term started to gain traction, quickly rising up a hype cycle aided by a strong boosterist discourse that contended big data was set to revolutionize how business is conducted and governance enacted. Not long after, the term drifted into common academic use accompanied by an argument that big data would transform how research would be conducted. [... ] big data and its associated data analytics represent a genuine revolution with respect to how we make sense of the world or whether it has been over-hyped and is merely a new inclusion amongst a suite of options in the academy's research armoury (Kitchin, 2017, S. 27 f.). 
Kitchin setzt sich damit auseinander, inwiefern sich Big Data von den Datensätzen unterscheidet, mit denen die sozialwissenschaftliche Forschung bislang gearbeitet hat und die die Informatik „Small Data“ nennt. ${ }^{14}$ Letztlich entfaltet er

[... ] the argument that it is leading to the creation of new research paradigms across disciplines, what have been termed data-driven science, computational social science and digital humanities. In particular, it focuses on the extent to which social media data, in combination with other big social data, offers the possibility for a different kind of social science (Kitchin, 2017, S. 28).

Laut Kitchin sind mit der Entwicklung von Big Data zugleich neue Analysemethoden aufgekommen; mithilfe maschineller Lernverfahren ziehen diese Erkenntnisse aus der „Datenflut“. Drei Gründe bedingen gemäß Kitchin den „Hype“ um die neue Art der Datenanalyse und markieren den zentralen Unterschied zu bisherigen Methoden. Die neuen Verfahren könnten (1) mit einer Flut von Daten variabler Qualität umgehen, (2) Daten auf innovative Art verwenden, Muster erkennen und Hypothesen generieren sowie (3) in beliebiger Vielzahl miteinander kombiniert und ferner zur Bestimmung des besten Erklärungsmodells auf einen Datensatz angewendet werden. Zusammengefasst spricht Kitchin von einem „Paradigmenwechsel“ und prognostiziert die Etablierung einer neuen Epistemologie über unterschiedliche Disziplinen hinweg. Dabei beruft er sich zunächst auf die 1962 von Thomas S. Kuhn vorgelegte Arbeit The Structure of Scientific Revolutions (1962/1996), die Paradigmenwechsel in der Wissenschaft damit erklärt, dass deren dominierender Modus bestimmte Phänomene nicht berücksichtigt oder Schlüsselfragen nicht beantwortet. Letztlich hält Kitchin aber die von Jim Gray im Jahr 2007 geäußerte Erklärung der gegenwärtigen „Datenrevolution“ für geeigneter im Hinblick auf den von ihm festgestellten Paradigmenwechsel: ${ }^{15}$

In contrast, Jim Gray [. . . ] proposed that the transitions between paradigms can also be founded on advances in data production and the development of new analytical methods. [...] Gray thus proposed that science was entering a fourth paradigm (exploratory science) based on the growing availability of big data and new analytics (his first paradigm was 'experimental science' that operated pre-Renaissance, the second was 'theoretical science' operating pre-computers, and the third was 'computational science' operating pre-big data) (Kitchin, 2017, S. 30).

\footnotetext{
${ }^{14}$ Aufgrund forschungsökonomischer und -strategischer Erwägungen kann darauf im Rahmen der hier vorliegenden Arbeit nicht näher eingegangen werden. Siehe hierzu: Kitchin, 2017, S. 28 ff..

${ }^{15}$ Kitchin bezieht sich hier auf den Beitrag Jim Gray on eScience. A Transformed Scientific Method von Hey, Tansley und Tolle (2009); dieser basiert auf dem Transskript eines Interviews, das der Informatiker Jim Grey in 2007 gegeben hat.
} 
Beruhend auf dieser Begründung untersucht Kitchin die drei folgenden Entwicklungen, auf die hier noch etwas genauer eingegangen wird: (1) Die Annahme, dass Big Data zugleich das ,Ende der Theorie' bedeute und einer neuen Art des Empirismus oder der Wissensproduktion den Weg bereite, in der Daten für sich selbst sprechen können; (2) die Schaffung einer datengetriebenen anstelle einer wissensbasierten Wissenschaft; (3) das Entstehen der Digital Humanities und Computational Social Sciences.

(1) Arbeiten, welche die „These vom Ende der Theorie“ aufgrund von Big Data stützen, suggerieren, dass ein neuer Wissenschaftsmodus mit rein induktiver Vorgehensweise geschaffen wird. Obwohl eine solche empirische Epistemologie laut Kitchin zunächst attraktiv wirkt, beruht diese auf einem Irrglauben in vier Punkten: (A) Big Data ist nicht erschöpfend; es handelt sich um eine Stichprobe, die durch die genutzte Technologie und Plattform, das verwendete Datenmaterial und auch das regulatorische Umfeld geprägt ist und damit einer Verzerrung unterliegt. (B) Big Data entsteht nicht aus dem „Nichts“; die Datensammlung erfolgt über diverse Systeme, die genutzten Analysen und Algorithmen beruhen auf wissenschaftlichen Überlegungen und werden durch entsprechende Tests verfeinert. (C) Ebenso wenig wie Daten frei von Theorie generiert werden, können diese für sich selbst sprechen, denn Daten beruhen auf menschlichen Vorurteilen oder Rahmungen; zur Sinnrekonstruktion müssen Daten interpretiert werden und auch dies geschieht nicht gänzlich objektiv, sondern aus einer bestimmten Perspektive heraus. (D) Daten können ohne Einbezug ihres Kontexts und frei von domänenspezifischer Expertise interpretiert werden; eine solche Interpretation ist jedoch ,,anämisch“ und hat wenig Nutzen, da die Einbettung in die breitere Debatte und in Wissen fehlt.

(2) Die Entwicklung der datengetriebenen Wissenschaft hält, so Kitchin, im Gegensatz dazu an Grundsätzen der wissenschaftlichen Methode fest, ist aber, um das Verständnis eines Phänomens zu fördern, offener für eine ,hybride Kombination" von abduktiven, induktiven und deduktiven Ansätzen. Kennzeichen der datengetriebenen Wissenschaft sei ein neuer Modus der Hypothesengenerierung; mit Techniken der Wissensfindung würden Fragestellungen für Untersuchungen identifiziert. Gesteuert würde der Prozess der Wissensentdeckung anhand von bestehender Theorie. Aus diesem Grund begreift Kitchin datengetriebene Wissenschaft auch als ,rekonfigurierte Version“ traditioneller wissenschaftlicher Methoden, die auf neue Weise Theorie bildet, und bewertet diesen erkenntnistheoretischen Wandel als überaus bedeutsam. Da sie Erkenntnisse generiere, welche die traditionelle wissensbasierte Wissenschaft nicht liefern kann, werde die datengetriebene Wissenschaft im Zeitalter von Big Data mitunter als neues Paradigma der wissenschaftlichen Methodik gehandelt. 
(3) In Bezug auf die Entwicklung des Entstehens der Digital Humanities oder auch Computational Social Sciences betont Kitchin den Unterschied zwischen den Natur- und Ingenieurwissenschaften gegenüber den Geistes- und Sozialwissenschaften. Die Epistemologie der Big-Data-Forschung oder datengetriebenen Wissenschaft scheine die Forschungsansätze der Erstgenannten bereits durchdrungen zu haben; im Gegensatz dazu sei die Entwicklung innerhalb dieser noch ungewiss. Diese Ungewissheit sieht Kitchin darin begründet, dass die Geistesund Sozialwissenschaften sehr vielfältige philosophische Grundlagen haben; beispielsweise biete Big Data für eine quantitativ orientierte Sozialforschung großes Potential. Insgesamt erkennt Kitchin im Entstehen der Digital Humanities und Computational Social Sciences Chancen wie Risiken:

For post-positivist scholars, big data offers both opportunities and challenges. The opportunities are a proliferation, digitization and interlinking of a diverse set of analogue and unstructured data, much of it new (e. g., social media) and many of which have heretofore been difficult to access (e. g., millions of books, documents, newspapers, photographs, art works, material objects, etc.) from across history that have been rendered into digital form over the past couple of decades by a range of organizations (Cohen, 2008); and the provision of new tools of data curation, management and analysis that can handle massive numbers of data objects. [. . . ] These opportunities are most widely being examined through the emerging field of digital humanities. [. . . ] The same kinds of argument can be levelled at computational social science. [. . . ] In other words, it is possible to think of new epistemologies that do not dismiss or reject big data analytics, but rather employ the methodological approach of datadriven science within a different epistemological framing that enables social scientists to draw valuable insights from big data (Kitchin, 2017, S. 33 f.).

Im Zuge seiner Auseinandersetzung mit der Frage, ob es sich bei Big Data nur um einen Hype oder doch um eine Revolution handelt, thematisiert Kitchin auch die Grenzen von Big Data. Er preist Small Data und spannt den Bogen von den Digital Humanities und Computational Social Sciences zur qualitativen Sozialforschung:

In general terms, it has been intimated that big data does represent a revolution in measurement that will inevitably lead to a revolution in how academic research is conducted; that big data studies will replace small data ones. However, this is unlikely to be the case for a number of reasons (Kitchin, 2017, S. 34). 
Mit den folgenden beiden Argumenten spricht sich Kitchin für die Koexistenz von Big Data Studies und Small Data Studies aus: (1) Small Data Studies könnten besser angepasst werden, um spezifische Forschungsfragen zu beantworten und vielfältige kontextuelle, rationale und irrationale Möglichkeiten, wie Menschen interagieren, die Welt verstehen und letztlich auch wie Prozesse funktionieren, im Detail zu erforschen; sie würden die Konzentration auf wenige Fälle ermöglichen, um anhand dieser individuelle, nuancierte und kontextbezogene Geschichten zu erzählen. (2) Big Data Studies würden keine tiefen Einblicke, sondern Momentaufnahmen liefern, aber oft zur Beantwortung von Fragen herangezogen, für die sie nie entwickelt wurden. Da Big Data Studies auf Stichproben beruhen, seien sie nicht erschöpfend, sondern von diversen Selektionskriterien bestimmt, wie zum Beispiel der Plattform und Technologie, dem Entstehungskontext oder dem regulatorischen Umfeld. Ferner würden Big Data Studies nur das erfassen, was offen ausgedrückt wird. Insgesamt assoziiert Kitchin Small Data Studies mit einem „Schürfen von Gold in einem schmalen Flöz“, Big Data Studies hingegen vergleicht er mit dem ,Versuch des Förderns von Goldklumpen im Tagebau durch das Schaufeln und Sieben riesiger Landstriche“:

\begin{abstract}
Small data studies then mine gold from working a narrow seam, whereas big data studies seek to extract nuggets through open-pit mining, scooping up and sieving huge tracts of land. These two approaches of narrow versus open mining have consequences with respect to data quality, fidelity and lineage. Given the limited sample sizes of small data, data quality - how clean (error and gap free), objective (bias free) and consistent (few discrepancies) the data are; veracity - the authenticity of the data and the extent to which they accurately (precision) and faithfully (fidelity, reliability) represent what they are meant to; and lineage - documentation that establishes provenance and fit for use; are of paramount importance [... ] (Kitchin, 2017, S. 35).
\end{abstract}

Kitchin stellt Small Data Studies und Big Data Studies einander gegenüber und hält fest, dass Erstere wegen der jeweiligen relativen Stärken und Grenzen und trotz der Vorteile, die mit Big Data und Social Media einhergehen, weiterhin ein wesentliches Element in der Forschungslandschaft darstellen werden. Es sei jedoch zu beachten, dass Small Data Studies verstärkt unter Druck geraten, neue Archivierungstechnologien zu nutzen; Daten werden, so Kitchin, in der digitalen Infrastruktur derart skaliert, dass sie für zukünftige Generationen erhalten bleiben sowie für eine Wiederverwendung und Kombination mit anderen Daten zugänglich werden, um Erkenntnisse durch den Einsatz von Big Data Analysen zu gewinnen. Insgesamt kommt Kitchin zu folgendem Schluss: 
There is little doubt that much of the rhetoric concerning big data is hyped and is boosterist, especially that produced by companies seeking to push new big data products, or research centres seeking to capture grant income. At the same time, there is no doubt that big data are qualitatively different to traditional small data and it does offer the potential to change how business is conducted, societies are governed, and academic research conducted. Big data and new data analytics do offer the possibility of reframing the epistemology of science, social science and humanities (though it will not lead to the 'end of theory'), and such a reframing is already actively taking place across disciplines. Nonetheless, small data studies will continue to be valuable because they have a tried and tested track record of producing insights by working a narrow seam and due to the various shortcomings of big data. As such, one can argue that there is a revolution underway, and that it will have profound effects, but that it will not lead to full-scale regime change. With respect to social media data then, its analysis will no doubt have a strong and positive impact on sociological and geographical research, providing a very rich, extensive, longitudinal set of data and studies, but these are most likely to complementary to a plethora of other studies (Kitchin, 2017, S. 36 f.).

Mit seinem Befund hat Kitchin, unter Einnahme einer Perspektive, die der Relevanz von Online-Forschung und sozialen Medien Rechnung trägt, eine Gegenwartsdiagnose der aktuellen Entwicklung sozialwissenschaftlicher Methoden vorgelegt. Mit ihren Big Data und Small Data Studies sind die Computational Social Sciences und Digital Humanities ein bedeutsamer Aspekt dieser. ${ }^{16} 17$

\footnotetext{
${ }^{16}$ Längst diskutieren die Computational Social Sciences oder Digital Humanities, zum Beispiel in Person von Latzko-Toth, Bonneau und Millette (2017), nicht mehr nur Big Data Studies und Small Data Studies, sondern setzen sich zudem mit der Data Thickness auseinander. Grund hierfür ist, dass die Qualität von Social-Media-Analysen oft einzig von der Quantität der untersuchten Daten abhängig gemacht wurde; Datenfülle galt als Garant für die Erfassung der sozialen, Wirklichkeit'. Thickening the Data beschreibt den Prozess, innerhalb dessen die Breite der Daten reduziert, dafür aber deren Tiefe erhöht wird. Anhand des Begriffs „Thick Data“ wird die nachhaltige Relevanz qualitativer Verfahren in der „Era of Big Data“ diskutiert.

${ }^{17}$ Die Beschäftigung mit der Kooperation von empirischer Sozialforschung und Informatik hat deutlich zugenommen; unterschiedliche Disziplinen betrachten seit einigen Jahren die methodologischen Aspekte von Social-Media-Analysen aus ihren je eigenen Perspektiven. Forschungsökonomische und -strategische Erwägungen bedingen es, dass im Rahmen dieser Arbeit, die den Sozialwissenschaften zuzurechnen ist, nicht weiter auf die Diskussion anderer Fachrichtungen eingegangen wird. Ebenso wenig kann sämtliche, sozialwissenschaftliche Literatur gewürdigt werden. Einige Literaturhinweise gibt diese Arbeit dennoch: Sarprasatham (2017) diskutiert Social-Media-Analysen im Zusammenhang mit Big Data aus einer wirtschaftswissenschaftlichen Perspektive und fokussiert auf Marketing. Mehrere Beiträge verschiedener Disziplinen enthält der Sammelband Debates in the Digital Humanities von Gold (2012). Außerdem sind die aktuelleren Sammelbände von Hai-Jew (2017d) sowie Stuetzer, Welker und Egger (2018) anzuführen.
} 


\subsection{Methodologische Besonderheiten und ethische Aspekte}

Mit dem Aufkommen von Social-Media-Analysen ist auch die Diskussion über die Potentiale und Herausforderungen, die die Methode für Wissenschaft und Wirtschaft bereithält, entfacht. Der Ruf nach einer systematischen Beschäftigung mit den Chancen und Risiken, die mit Social-Media-Analysen einhergehen, sowie einer Reflexion dieser ist laut geworden. Mittlerweile wurde die Methode schon an verschiedener Stelle und in heterogenem Kontext diskutiert. Auch diese Arbeit hat zwar bereits einige Spezifika der Methode erwähnt, doch eine umfassende theoretischen Betrachtung von Social-Media-Analysen verlangt danach, dass relevante methodologische Besonderheiten und ethische Aspekte im Hinblick auf die Methode noch einmal gesondert dargelegt werden. Dies geschieht nun anhand von zwei Artikeln, welche eben diese in ihren Mittelpunkt rücken und zudem die zentralen Vor- und Nachteile der Methode betrachten.

Sudulich et al. (2014) begegnen der empirischen Sozialforschung, die sich des Internets bedient, mit einem methodologischen Blick; sie umreißen das Gros der für Social-Media-Analysen als Methode relevanten theoretischen Grundlagen. Wie viele andere Autoren sprechen auch sie von einer wachsenden sozialen und politischen Bedeutung des Web 2.0 in der Gegenwart. Sie nennen das Web 2.0 ein „politisches Internet“, was ihrem politikwissenschaftlichen Hintergrund geschuldet scheint. Obwohl sie einige Risiken für die Sozialforschung erkennen, sehen sie durchaus auch zahlreiche Chancen:

We argue that three key characteristics of online political information in the Web 2.0 era shape and constrain any study of the political Internet. These characteristics are (1) extremely large volume, (2) heterogeneity and (3) plasticity. We contend that this combination creates what we term a 'dynamic data deluge' for social scientists, which makes distinguishing and recording meaningful information generated by the political Internet a methodologically challenging endeavour (Sudulich et al., 2014, S. 1).

Sudulich et al. fordern von der Sozialforschung methodologische Offenheit; eine solche würde es ermöglichen öffentliches Wissen, das über das globale und multifunktionale Medium Internet zugänglich wird, zu akkumulieren. Transparente wie reproduzierbare Methoden seien zu entwickeln, um darüber systematisch das Verständnis des Internets zu erhöhen; etablierte Methoden empirischer Sozialforschung gelte es zu diesem Zweck ebenso zu nutzen wie die noch im Entstehen 
begriffenen Methoden zur Erforschung der Online-Umwelt. Hier bestehe Handlungsbedarf, denn

[... ] this progression has occurred with relatively little methodological introspection, a lacuna that is all the more keenly felt given the unique challenges and opportunities that data generated by the 'Web 2.0' or social media Internet offers to researchers (Sudulich et al., 2014, S. 3).

Daher sind für eine empirische Sozialforschung, welche die Dynamik und die Prozesse des Internets zu erklären beabsichtigt, nach Ansicht von Sudulich et al. entsprechende methodologische Überlegungen zwingend erforderlich:

The Internet is an environment where 'everything flows' at an unprecedented pace, with content and connections constantly being created, amended and deleted. There is thus an emerging disjuncture between the Internet's permanence as an element of social (and political) life and the large-scale, diverse and highly ephemeral nature of its content (Sudulich et al., 2014, S. 5).

Methodologische Reflexion bedeute, die drei dem Internet inhärenten Charakteristika bei jeder einzelnen Social-Media-Analyse mit zu bedenken; im Hinblick auf empirische Untersuchungen seien diese von signifikanter Bedeutung. Nach Sudulich et al. handelt es sich dabei um (1) die Unmenge an Informationen, (2) deren Heterogenität und (3) die Plastizität der Inhalte.

(1) Die Unmenge an Informationen, die im Internet verfügbar ist, und laut Sudulich et al. das erste entscheidende Charakteristikum des Internets markiert, wird meist als Big Data diskutiert. Methodologisch impliziert Big Data für die empirische Sozialforschung, wie zuvor schon mit Kitchin (2017) gezeigt, dass das Internet einen großen Datenkorpus generiert. Zwar beinhaltet dieser nicht selten bedeutungslose Daten, „Noise“ oder auch „Rauschen“ genannt, das mitunter von Bots erzeugt, also nicht von Menschenhand, sondern automatisiert hergestellt wird, doch ebenso sind bedeutsame Informationen vorhanden. ${ }^{18}$ Das Vorliegen der Daten in digitaler Form ermöglicht, so Sudulich et al., deren Sammeln und die Reflexion in Bezug auf den originalen Inhalt:

The Internet generates an enormous corpus of politically relevant information, and the digital quality of this information means that it can be captured to faithfully reflect its original content (Sudulich et al., 2014, S. 9).

${ }^{18}$ Bots beziehungsweise automatisierte Konten sozialer Medien und Techniken, um Bots aufzudecken, thematisiert zum Beispiel Hai-Jew (2017a). Eine kritische Reflexion in Bezug auf Bots findet sich ferner bei Tuten und Solomon (2018, S. 113 f.). 
Allerdings gelte es zu beachten, dass mit den Ergebnissen von Social-MediaAnalysen stets kritisch umzugehen ist; neben dem Risiko der Datengenerierung durch Bots sei die besondere Qualität der den sozialen Medien entstammenden Daten zu bedenken. Im Hinblick auf diese kann beispielsweise auch erneut auf die zuvor bereits angesprochene digitale Spaltung verwiesen werden; methodologisch impliziert diese, so Sudulich et al., eine systematische Verzerrung der Ergebnisse und mindert damit deren Eignung für die Prognose gesellschaftlicher Trends und gesamtgesellschaftliche Verallgemeinerungen:

The methodological question of interest is whether the large volume of data generated online can be leveraged in order to produce either transformed datasets or sub-samples that can be used to make inferences about out-of-sample populations (Sudulich et al., 2014, S. 11).

Daher bewertet Kitchin (2017) Social-Media-Analysen als „nicht erschöpfend“.

(2) Die Heterogenität oder Diversität der Daten sozialer Medien geben Sudulich et al. als das zweite wesentliche Charakteristikum des Internets an. Daten existieren als Text oder Graphik beziehungsweise Bild, im Audio- sowie Video-Format. Methodologisch verlange diese Vielfalt an Formaten nach neuen methodischen Werkzeugen:

In order to capture such a diverse mass of content, new tools have to be designed to fit the complexity of the object of study. [... ] Social media offer new global and aggregate sources of data on popular behaviour and trends. Search tools, links, comments and 'like buttons' on social network profiles all present new means of measuring popularity and public interest in people, organisations and events. Tracing patterns of interaction and connection through hyperlinks and Twitter or YouTube networks [. . . ] can open up the 'weak ties' that bind individuals to a cause or movement (Sudulich et al., 2014, S. 11).

Im Hinblick auf Social-Media-Analysen unterscheiden Sudulich et al. Netzwerkund Inhaltsanalysen. Letztere würden für die Sozialforschung kein mit dem Internet oder sozialen Medien einhergehendes methodisches Novum markieren, sondern seien als „Prozess des Extrahierens bedeutungsvoller Informationen aus Daten im Textformat" eine zentrale Herausforderung einer Sozialforschung, die mit dem Internet arbeitet. Laut Sudulich et al. handelt es sich bei Inhaltsanalysen um eine der mächtigsten Techniken zur Ableitung von essentiellen Generalisierungen aus Daten. Im Hinblick auf Social-Media-Analysen gelte es vor allem bezüglich der Reliabilität und Validität zwischen einer manuellen und automatisierten Codierung zu unterscheiden: 
Whereas computerised techniques can claim perfect reliability, human coding provides a higher level of validity, as human coders with an awareness of the context can make inferences about content that are (currently) impossible for machines to reach. [... ] Automated coding requires a certain structure and volume of textual data and can be somewhat ambiguous in its output - often requiring subjective substantive interpretation to be usable as a source of meaningful political analysis. While of course this doesn't mean that human coding is precluded for digital data [. . . ], it does mean that a bias towards more automation is inevitably introduced to minimise loss of information. One potentially promising avenue of research that seeks to address this trade-off is the crowd sourcing of data coding, with large numbers of non-expert coders being used either alongside or instead of automated coding for large volumes of political text [... ]. However, with large-scale textual data generated online, human coding is increasingly used as a post-hoc check or confirmatory exercise on the results derived from computer-based methods (Sudulich et al., 2014, S. 13).

Insgesamt erkennen Sudulich et al. drei elementare Charakteristika des Internets als in methodologischer Hinsicht bedeutsam. (3) Dessen Plastizität wird auch als „dynamische Natur“ beschrieben:

The web, unlike previous electronic and print-based forms of media, is an everchanging space. Much of the data that it generates are ephemeral and can be subject to almost instantaneous change. [. . . ] [T]he transience of the Internet as a medium involves two dimensions: content and construction (Sudulich et al., 2014, S. 14).

Inhalte im Internet können, so Sudulich et al., mitunter schon nach kurzer Zeit wieder verschwunden sein und dann nicht über einen langen Zeitraum zurückverfolgt werden. Werden solche Daten anfangs nicht miterhoben, sind diese unwiederbringlich verloren. Dies könne gravierende Folgen für die Ergebnisse von Social-Media-Analysen haben und zu deren Verzerrung oder auch Ungültigkeit führen. Hinzu komme, dass die Inhalte des Internets stets rekonstruiert werden müssen; jedoch könnten soziale Medien nicht als konstant angesehen werden. Eine Rekonstruktion gesammelter Inhalte erfolgt laut Sudulich et al. oft nicht geradlinig über einen längeren Zeitraum. Außerdem könnten Veränderungen im Datenformat oder auch der Software die Rekonstruktion der zuvor gesammelten Daten negativ beeinflussen.

Sudulich et al. kommen zu dem Schluss, dass trotz der thematisierten methodologischen Herausforderungen auch erhebliche Potentiale mit Social-MediaAnalysen einhergehen. Anhand sozialer Medien könnten Phänomene erforscht werden, die zuvor nicht greifbar waren. Die drei von ihnen diskutierten, das Internet aus methodologischer Sicht der Sozialforschung ausmachenden Charakteristika hätten jedoch bedeutende Folgen im Hinblick auf das Forschungsdesign und die Schritte im Forschungsprozess, wie die Auswahl und Sammlung der Daten und schließlich auch deren Analyse: 
We argue that moving forward with the task of extracting meaningful information from web-based data requires a radical re-thinking of the optimal manner of approaching the research design process. Specifically, we need to revisit the choice between inductive and deductive approaches (Sudulich et al., 2014, S. 15).

Nach Ansicht von Sudulich et al. ist es daher an der Zeit, die Eignung deduktiver und damit theoriegeleiteter gegenüber induktiven und damit offenen Ansätzen, die sich nicht an Hypothesen, sondern an den Daten selbst und deren ,Beobachtung ‘ orientieren, also explorativ vorgehen, für Social-Media-Analysen zu reflektieren:

It should be acknowledged that modern political science is dominated by the Popperian 'positivist' approach. In line with this tradition, most studies of the political Internet have adopted a deductive approach, based on the classification and interpretation of web content on the basis of theoretical speculations. While this logic deeply characterises social science research, its application to web-originated data is somewhat more problematic than to other type of data. This is principally due to what we describe above as the 'plasticity' of online data. As we outlined above, the Internet and associated technologies have rapidly changed during its relatively short life as a mass medium and, the audience using these technologies has expanded rapidly. While a hypothesis may be valid at one point in the development of the Internet as a medium, there is no guarantee that it will remain so for a significant amount of time. Furthermore, certain hypotheses may only emerge in the light of fresh technological or audience developments while others may cease to make sense. [. . . ] [T] his rapid and continuous changes in both the audiences using online technologies and the types of participation and interaction that those technologies offer - combined with a relatively glacial grant application, research and publication cycle - means that many research findings about the political Internet are 'rendered obsolete by the time that they are published'. These considerations would point towards an indicative and highly conditional approach to the study of the political Internet. However, such an approach comes with associated costs. For instance, the lack of universally accepted theoretical classifications of online politically relevant material has produced a multiplicity of rather inconsistent empirical methods of data classification and analysis. [... ] [T] he field has deviated from such a template in a rather chaotic fashion, which makes cross-country and longitudinal comparison extremely difficult. We ought to regard such a lack of consistency as a shortcoming of inductive and ad hoc theoretical and methodological development in the study of the political Internet (Sudulich et al., 2014, S. 16 f.).

Neben Sudulich et al. betrachten noch einige andere Autoren Social-MediaAnalysen aus einer methodologischen Perspektive; so auch McCay-Peet und Quan-Haase (2017), die zwar ähnliche Aspekte wie Sudulich et al. nennen, aber auch ergänzen: 
What makes the study of social media relevant to many disciplines is the availability of vast amounts of varied data. Social media produces what has been referred to as big data and is characterized by high velocity, large volume, diverse variety, exhaustivity in scope, fine-grained resolution, relational in nature, and flexibility in its approach [...]. This creates new challenges for scholars, while also presenting great opportunity - this has given rise to several questions (McCay-Peet, \& Quan-Haase, 2017, S. 19).

Einerseits sehen McCay-Peet und Quan-Haase, dass Social-Media-Forschung Fragen aufgeworfen hat, die die Forscher zwingen, ,in diese hineinzublicken“ und sich mit den der Social-Media-Forschung inhärenten Herausforderungen auseinanderzusetzen; hierzu werden methodologische und ethische Fragen sowie Fragen in Bezug auf die Skalierung gezählt. Erstere wurden mit Sudulich et al. bereits behandelt; McCay-Peet und Quan-Haase legen hierzu keine nennenswerten Ergänzungen vor. Jedoch betrachten sie zusätzlich ethische Fragen, was mitunter damit zusammenhängt, dass diese vielerorts inzwischen in den Fokus der methodologischen Betrachtung gerückt sind:

Data collection, aggregation, and reporting of social media data has raised numerous ethical questions relating to issues such as personal privacy, accuracy, and accountability with which researchers and practitioners are only beginning to grapple. While social media data is often publicly available, there are still many ethical considerations that should give researchers reason to pause (McCay-Peet, \& Quan-Haase, 2017, S. 19).

Es herrsche in der Diskussion ethischer Aspekte von Social-Media-Analysen Einigkeit darin, dass nicht sämtliche Nutzer sozialer Medien mit dem Gebrauch ihrer Daten für Analysezwecke einverstanden oder sich dessen gar nicht bewusst sind. Jedoch sind, so betonen McCay-Peet und Quan-Haase, ethische Bedenken nicht nur im Kontext von Big Data Scarping relevant, sondern auch bei Untersuchungen mit kleiner Grundgesamtheit. Vor allem bei qualitativen Untersuchungen mit kleiner Stichprobe sei die Identifikation einzelner Nutzer wesentlich einfacher. Aus diesem Grund müsse sich die Social-Media-Forschung mit etlichen Fragen rund um das Thema „Datensicherheit“" beziehungsweise dem verantwortungsvollen Umgang mit Daten beschäftigen; es gelte zu klären, welche ethischen Richtlinien aufgestellt werden müssen, um zugleich der Wissenschaft zu neuen Erkenntnissen zu verhelfen und das Recht der Nutzer auf Datenschutz zu respektieren. ${ }^{19}$ Mit Fragen zur Skalierung der Social-Media-Forschung halten

\footnotetext{
${ }^{19}$ Teilweise hängen ethische Aspekte eng mit rechtlichen zusammen, wie beim Thema „Datenschutz". Aus forschungsstrategischen wie -ökonomischen Gründen geht diese Studie nicht darauf ein. Social Media als Gegenstand und Instrument der Forschung werden aus juristischer Perspektive ausführlich von Tscherwinka (2014) behandelt.
} 
McCay-Peet und Quan-Haase, ähnlich wie Kitchin (2017), fest, dass SocialMedia-Analysen auf zwei Arten angelegt sein können: Als quantitative und als qualitative Untersuchung, mit entsprechend größerer oder kleinerer Stichprobe. Beide Ansätze werden als relevant und valide bewertet, doch ebenso neue theoretische und methodologische Überlegungen in Bezug auf deren Kombination und Integration gefordert:

\begin{abstract}
A study can rely on either large data sets that aggregate terabytes of information or, through small-scale studies, examine the local behaviour of a few users. While both approaches are relevant and valid, they provide qualitatively different insights into a single phenomenon. So, how do we integrate findings from such disparate means of gaining knowledge? New theoretical and methodological assumptions are needed to link and integrate distinct data sets and findings (McCay-Peet, \& Quan-Haase, 2017, S. 19).
\end{abstract}

Andererseits thematisieren McCay-Peet und Quan-Haase „Forschung mit Social Media" und erkennen in den Daten sozialer Medien neben den der SocialMedia-Forschung inhärenten Herausforderungen, die Möglichkeiten, neue Fragestellungen zu verfolgen sowie bestehende Forschungsprobleme aus einem anderen Blickwinkel zu betrachten. Hierbei handelt es sich um Fragen, die die Nutzung sozialer Medien selbst betreffen, sowie um solche, deren Beantwortung dem Verständnis sozialer Phänomene dient. Bei Ersteren stünden Aktivitäten in Social Media im Fokus der Betrachtung. Organisationen müssten soziale Medien verstehen, um Strategien zur Unterstützung sowie zur Kontrolle ihrer Nutzung zu entwickeln. Bei Letzteren geht es um Social-Media-Analysen als neue Methode im Repertoire der Sozialforschung:

A second stream of social media research is the use of social media as a tool or method for academic research, for examining research questions and understanding complex problems otherwise examined through other, more traditional, methods (McCay-Peet, \& Quan-Haase, 2017, S. 20).

McCay-Peet und Quan-Haase zeigen die Chancen, die Social-Media-Analysen bieten, weisen jedoch ebenso auf die mit diesen für die Forschung einhergehenden Risiken hin. Dies sei das Verkennen der Tatsache, dass Nutzer mehrere soziale Medien oft parallel gebrauchen; verschiedene soziale Medien bedienen, so McCay-Peet und Quan-Haase, unterschiedliche Bedürfnisse. Ferner wird die Annahme gerügt, dass Social Media und die darin auftretenden Phänomene isoliert von anderen Lebensbereichen existieren. In Social-Media-Analysen gelte es den sozialen Kontext zu berücksichtigen: 
Hence social media needs to be studied as an expansion of daily life, a means to amplify social phenomena, and a catalyst for social phenomena in order to understand the larger ramifications of social media in society and points of intersection (McCay-Peet, \& Quan-Haase, 2017, S. 21).

Ferner empfehlen McCay-Peet und Quan-Haase eine fachliche Ausrichtung von Social-Media-Analysen und deren Einsatz für themenspezifische Fragestellungen; dies mache zum einen eine theoretische Verankerung der Forschung, zum anderen die Anpassung der Methode an spezifische Anforderungen möglich. Insgesamt nennen McCay-Peet und Quan-Haase sieben Beweggründe für das Engagement der Nutzer in Social Media und damit zugleich sieben Aspekte für die Ausrichtung von Social-Media-Analysen: (1) Presentation of Self, Reputation Management, and Privacy, (2) Action and Participation, (3) Uses and Gratifications, (4) Positive and Negative Experiences, (5) Usage and Activity Counts, (6) Social Context, (7) Platform Characteristics. ${ }^{20}$

McCay-Peet und Quan-Haase schließen mit der Prognose, dass die Entwicklung neuer Methoden für die Social-Media-Forschung zunehmen und mehr Geltung erlangen wird. Beide Tendenzen würden dazu beitragen, Methoden der Social-Media-Forschung und Wissen in Bezug auf diese in schon bestehende Rahmenbedingungen zu integrieren. Abschließend fordern allerdings auch McCay-Peet und Quan-Haase, wie dies bereits Sudulich et al. getan haben, mehr kritische Forschung zu methodologischen Grundlagen und ethischen Aspekten:

[. . . ] more critical research is needed to understand the biases inherent in using social media methodologies and to develop best practices concerning social media research, with the aim of both supporting researchers and protecting social media users. Of particular importance is the need to determine best practices around ethical considerations. [. . . ] There is much work to be done not only around the social phenomena under investigation on social media platforms, but also concerning how scholars are procuring, storing, interpreting, and making use of social media data (McCay-Peet, \& Quan-Haase, 2017, S. 21).

${ }^{20}$ Anhand einiger Beispiele für Forschungsfragen buchstabieren McCay-Peet und QuanHaase (2017, S. 21 f.) die von ihnen bestimmten sieben Kriterien der Nutzung von sozialen Medien aus. Diese scheinen selbsterklärend und werden daher und auch aus forschungsökonomischen wie -strategischen Gründen in dieser Arbeit nicht weiter vorgestellt. 


\subsection{Methodik von Social-Media-Analysen}

Social-Media-Analysen wurden bereits als eine neue, mit der Etablierung der sozialen Medien einhergehende methodische Entwicklung im Kontext der Computational Social Sciences und Digital Humanities thematisiert. Methodologische Besonderheiten und ethische Aspekte der Verfahren wurden ebenso behandelt. Es gilt nun den Blick noch einmal konkret auf die Methode der Social-MediaAnalysen zu richten. Der vorliegende Abschnitt zur Methodik von Social-MediaAnalysen zeigt den Status quo der Methode. Dabei richtet sich der Fokus auf soziale Medien als Instrument der Forschung; von der Betrachtung sozialer Medien als Gegenstand der Forschung wird abgesehen (König et al., 2014; Welker, \& Kloß, 2014; Zeller, 2017a, 2017b). ${ }^{21}$ Es sei vorangestellt, dass SocialMedia-Analysen eine heterogene, bisweilen auch diffuse Methode darstellen. Mitunter zeichnet sich diese dadurch aus, dass ihr Status quo aufgrund des rasanten Wandels, dem soziale Medien unterliegen, und des beständigen technologischen Fortschritts, der diesen Wandel mitbedingt, stets nur vorläufig und auf die Gegenwart bezogen sein kann. Im deutschen und auch englischen Sprachraum hat sich Frauke Zeller (2017a, 2017b) mit sozialen Medien innerhalb der empirischen Sozialforschung eingehend auseinandergesetzt. Mit ihren Beiträgen hat sie ein wesentliches Element für die theoretische Betrachtung von Social-MediaAnalysen vorgelegt. Daher wird deren Methodik in erster Linie anhand von zwei Arbeiten Zellers thematisiert. ${ }^{22} 23$

Gemeinhin gelten Social-Media-Analysen als eine neue und insbesondere im Hinblick auf ihre methodologische Reflexion noch in den Kinderschuhen steckende Variante der Online-Forschung. Zeller schlägt daher vor, etablierte

${ }^{21}$ Social Media als Forschungsgegenstand behandeln beispielsweise: Emmer, 2017; Kolo, 2017.

22 Von Seiten der Wirtschaftsinformatik haben Stieglitz, Dang-Xuan, Bruns und Neuberger (2014) einen Artikel über „Social Media Analytics“ vorgelegt; dieser bietet einen ersten Einstieg in deutscher Sprache. Da er inhaltlich jedoch keine ergänzenden Erkenntnisse zu den Beiträgen Zellers bereithält, einer für den Fokus dieser Arbeit nicht vordergründig relevanten Fachrichtung entstammt und daher auch nicht in die sozialwissenschaftlich relevanten Tiefen vorstößt, sei er hier nicht mehr als erwähnt.

${ }^{23}$ Ein sehr grober und wegen der Veröffentlichung in 2011 nicht mehr aktueller Überblick über Social-Media-Analysen findet sich unter dem Titel „Web Monitoring“ in dem Buch Social Web von Ebersbach, Glaser und Heigl (2011. S. 217 ff.). Zu erwähnen ist dieser aufgrund seiner Kürze und des Mangels an Tiefgang und vor allem, da der Überblick das Verständnis von Social-Media-Analysen widerspiegelt, das etliche Praktiker der Methode im Rahmen der empirischen Untersuchung dieser Arbeit gezeigt haben. 
Ansätze zu adaptieren und um Erkenntnisse der Big-Data-Forschung anzureichern. Forschung im Zusammenhang mit Social Media sei nicht nur mit technischen Anforderungen konfrontiert, sondern sehe sich ferner mit einem modifizierten Datenbegriff und Forschungsprozess konfrontiert. Geht es um soziale Medien als Forschungsinstrument, wird deren Nutzung laut Zeller zum Zweck der Erhebung oder Messung von bestimmten Aspekten betrachtet. Sowohl Onlineals auch Offline-Fragestellungen würden anhand sozialer Medien untersucht. Zeller bewertet soziale Medien als wirksames Instrument zur Informationsverarbeitung, Informationsbeschaffung und Datenerhebung. Unter Rekurs auf Robert Ackland (2013) sowie Martin Welker und Uwe Matzat (2009) merkt sie an, dass Online-Forschung in der Regel binär systematisiert wird und dies zudem in zweifacher Hinsicht: Zum einen in quantitative und qualitative Erhebungsund Auswertungsmethoden, zum anderen in reaktive und nicht-reaktive Verfahren der Datenerhebung. Social-Media-Analysen sind mit den Charakteristika sozialer Medien konfrontiert und setzen wegen der großen Menge an Rohdaten, die in ihrem Rahmen ausgewertet werden gemäß Zeller vor allem auf nicht-reaktive Verfahren (Ackland, 2013; Welker, \& Matzat, 2009; Welker et al., 2014; Zeller, 2017a, S. 390 f.; Zeller, 2017b, S. 387 f.). ${ }^{24}$

Wie in Abbildung 3.2 dargestellt, gibt Zeller einen die Erkenntnisse der universitären und kommerziellen Forschung integrierenden graphischen Überblick über die Varianten von Social-Media-Analysen; ihre kommunikationswissenschaftliche Perspektive, die ebenso als allgemein sozialwissenschaftliche gelesen werden kann, wird um diverse Ansätze benachbarter Disziplinen ergänzt. In Abbildung 3.2 werden über drei Zeilen quantitative und qualitative Verfahren sowie Methodenkombinationen unterschieden; zudem sind die drei Varianten durch eine weitere Spaltung der drei Zeilen in reaktive und nicht-reaktive Verfahren gegliedert, wobei Erstere auch interaktive Ansätze beinhalten. ${ }^{25}$ Des Weiteren sind die einzelnen Varianten von Social-Media-Analysen nicht nur in diese Zeilen einsortiert, sondern anhand von zwei Spalten ferner in empirische Methoden der Kommunikationswissenschaft und in von dieser beziehungsweise von der allgemeinen Sozialforschung adaptierte Methoden anderer Disziplinen systematisiert. Für die hier vorliegende Forschungsarbeit sind mit Blick auf die noch

${ }^{24}$ Graffigna und Riva (2015) betonen die Potentiale qualitativ ausgerichteter Social-MediaAnalysen sowie integrierten Methodenkombinationen und schlagen einen konkreten Ansatz vor.

${ }^{25}$ Interaktive Ansätze, wie Market Research Online Communities und Crowdsourcing, diskutiert vor allem die Marktforschung als vielversprechend. Vergleiche die im weiteren Verlauf behandelten Einsatzgebiete von Social-Media-Analysen, wie die Marktforschung im Web 2.0 (3.6). 


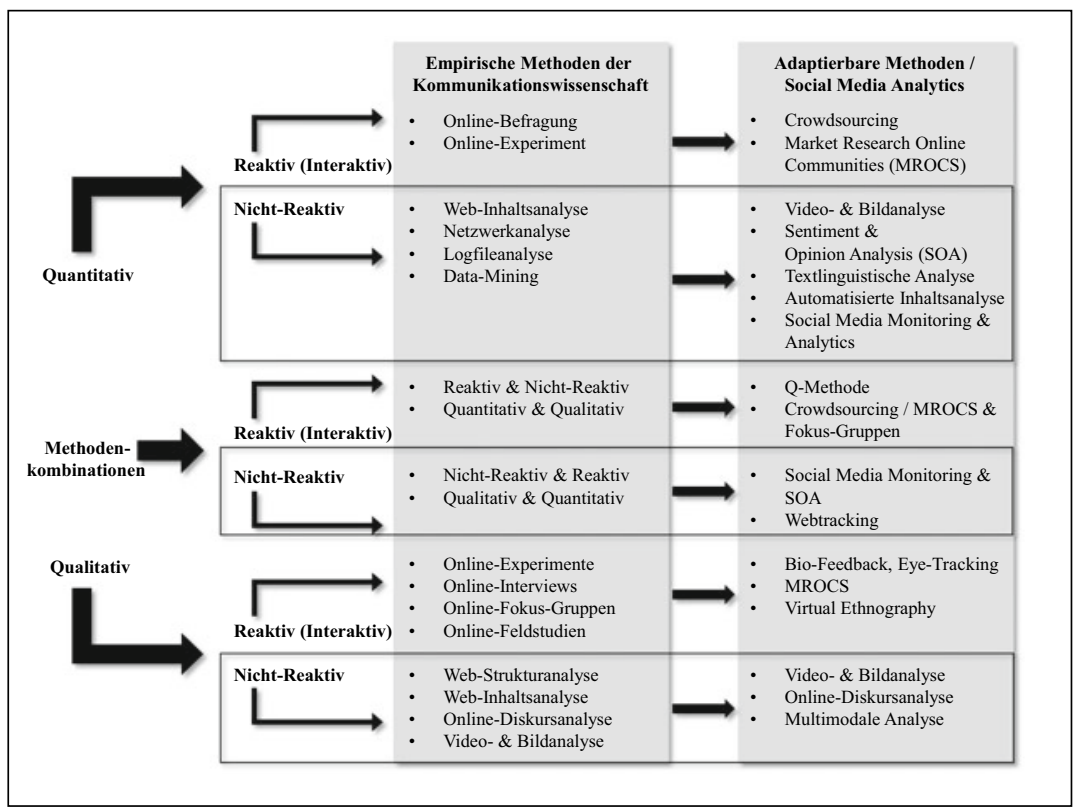

Abbildung 3.2 Social-Media-Analysen - Varianten (Zeller, 2017a, S. 392)

folgende Ergebnisdarstellung der empirischen Untersuchung insbesondere die nicht-reaktiven Verfahren, sowohl in quantitativer (1) als auch in qualitativer Ausprägung (2) sowie in ihrer Kombination (3) von Relevanz; daher werden diese nun näher betrachtet (Zeller, 2017a, S. 391 ff.; Zeller, 2017b, S. 391 ff.). ${ }^{26}$

(1) Quantitative, nicht-reaktive Methoden der Kommunikationswissenschaft umfassen nach Abbildung 3.2 mit Web-Inhaltsanalysen, Netzwerkanalysen, Logfileanalysen und Data Mining solche Verfahren, die zwar auf etablierten sozialwissenschaftlichen Methoden beruhen, aber des Einsatzes spezieller Software bedürfen. Zeller merkt an, dass Web-Inhaltsanalysen die bei klassischen quantitativen Inhaltsanalysen übliche Konzeption eines Codebuchs vorangehen sollte. Bei der Auswahl und auch der Nutzung einer Software, die für die Erhebung der benötigten Web-Inhalte herangezogen wird, seien die dieser mitunter inhärenten

${ }^{26}$ Aus forschungsökonomischen und -strategischen Gründen kann in dieser Arbeit nicht auf sämtliche in Abbildung 3.2 genannte Verfahren gesondert eingegangen werden. Siehe hierzu: Zeller, 2017, S. 393 ff.. 
Einschränkungen zu beachten; angesprochen sind beispielsweise Fragen in Bezug auf die Speicherung verschiedener Datenformate und, ob Software-Hyperlinks enthalten sind und wie diese dann kenntlich gemacht werden (Krippendorff, 2018; Herring, 2010; Vis, 2013; Zeller, 2017a, S. 393; Zeller, 2017b, S. 393). ${ }^{27}$

Quantitative, nicht-reaktive und von den Sozialwissenschaften adaptierte Methoden sind gemäß Abbildung 3.2 zunächst Video- \& Bildanalysen. Klassischerweise sind diese Verfahren qualitativ ausgerichtet, werden von Zeller aber wegen einer Beteiligung der Informatik als quantitativ klassifiziert. Hierzu zählen auch Ansätze, die die in sozialen Medien enthaltene Bilder und Videos automatisiert analysieren. Drei weitere Verfahren, die Zeller dieser Gruppe zurechnet und als Automatisierte Textanalysen zusammenfasst, sind Sentiment \& Opinion Analysis, Textlinguistische Analysen und Automatisierte Inhaltsanalysen. In erster Linie würden die drei Verfahren für große, Social Media entnommene Datensätze genutzt (Ghoshal, Ircing, \& Khudanpur, 2005; Goodrum, Devereaux, \& Smith, 2009; Lewis, Hermida, \& Zamith, 2013; Liu, 2015; Mehl, \& Gill, 2010; Scharkow, 2013; Tumasjan, Sprenger, Sandner, \& Welpe, 2010; Young, \& Soroka, 2012; Zeller, 2017a, S. 393 f.; Zeller, 2017b, S. 393). Social Media Monitoring \& Analytics ist das letzte Verfahren, das innerhalb des Blocks der quantitativen, nicht-reaktiven und von den Sozialwissenschaften adaptierten Methoden gelistet wird. Es entstammt laut Zeller der kommerziellen Marktforschung und weist eine Vielzahl an Ausprägungen auf, die in Abbildung 3.3 gesondert tabellarisiert sind (Zeller, 2017a, S. 394; Zeller, 2017b, S. 393 f.).

Laut Zeller ist es ausschlaggebend, dass alle Social Media Monitoring \& Analytics zuzurechnende Verfahren auf passiven Erhebungsmethoden und auch dem Zählen von Häufigkeiten beruhen. Nicht zu vernachlässigen sei in diesem Zusammenhang das Hinterfragen der Erhebung einzelner Daten und deren Repräsentativität; kommerzielle Anbieter würden oft keinen Einblick in Reichweite, Stichprobe und Repräsentativität gewähren (Scheffler, 2014; Zeller, 2017a, S. 394; Zeller, 2017b, S. 393f). ${ }^{28}$

\footnotetext{
${ }^{27} \mathrm{Zu}$ Netzwerkanalysen als Variante von Social-Media-Analysen liegen mehrere Arbeiten vor. Siehe zum Beispiel: Borgatti, Everett, \& Johnson, 2013; Fu, Luo, \& Boos, 2017; Scott, \& Carrington, 2011.

${ }^{28}$ Die Tabellarisierung von Social-Media-Analysen innerhalb der Marktforschung, wie sie Abbildung 3.3 zeigt, scheint selbsterklärend und wird daher im Rahmen dieser Studie nicht näher erläutert. Siehe hierzu: Zeller, 2017, S. 394 f..
} 


\begin{tabular}{|c|c|c|}
\hline & Nutzungsvariable & Indikator / Messung \\
\hline Monitoring & $\begin{array}{l}\text { Sichtbarkeit, Reichweite, } \\
\text { Aufmerksamkeit }\end{array}$ & $\begin{aligned}> & \text { Share of Buzz, Share of Voice } \\
> & \text { Anzahl Quellen / Posts } \\
> & \text { Anzahl Autoren } \\
> & \text { Anzahl positiver / negativer } \\
& \text { Nennungen }\end{aligned}$ \\
\hline Social Media Analytics & $\begin{array}{l}\text { Viralitäts- \& Einflusspotential von } \\
\text { Inhalt / Autoren / Kanälen }\end{array}$ & $\begin{aligned}> & \text { Vernetzungsgrade von Profilen, } \\
& \text { Weiterempfehlungen, Likes, } \\
& \text { Bewertungen } \\
> & \text { Interaktionsrate pro Post } \\
> & \text { Fanwachstum } \\
> & \text { Unique User / Views pro Kanal } \\
> & \text { Anzahl relevanter Fans }\end{aligned}$ \\
\hline Klassische Web-Analytics & Attraktivität des Inhalts / Autors & $\begin{array}{l}>\text { Webtracking } \\
>\text { Anzahl Website-Besucher } \\
\text { insgesamt } \\
>\text { Anteil Website-Besucher aus } \\
\text { Social Media generiert } \\
>\text { Verweildauer } \\
>\text { Sichtbarkeit }\end{array}$ \\
\hline
\end{tabular}

Abbildung 3.3 Social-Media-Analysen - Einsatzgebiet: Marktforschung (Zeller, 2017a, S. 394)

(2) Neben dem soeben bereits als nicht-reaktives, quantitatives Verfahren thematisierten Social Media Monitoring und der zuvor auch schon erwähnten Sentiment \& Opinion Analysis führt Zeller Webtracking als ein weiteres nicht-reaktives Verfahren im Bereich der Methodenkombinationen an. Es handelt sich dabei um eine Methode, die über in Webseiten integrierten Algorithmen deren Besucher registriert. Webtracking wird heute verstärkt hinsichtlich ethischer Aspekte diskutiert (Mayer, \& Mitchell, 2012; Zeller, 2017a, S. 396; Zeller, 2017b, S. 396).

(3) Neben quantitativen Methoden und Methodenkombinationen beinhaltet Abbildung 3.2 ferner qualitative, nicht-reaktive Verfahren; konkret genannt werden aus dem Bereich der empirischen Kommunikationswissenschaft WebStrukturanalysen, erneut Web-Inhaltsanalysen, Online-Diskursanalysen und die auch schon erwähnten Video- \& Bildanalysen. Die beiden zuletzt angeführten werden auch als von der Sozialforschung adaptierte Verfahren gelistet und hier noch um Multimodale Analysen ergänzt. Web-Strukturanalysen kommen in der Online-Community-Forschung zum Einsatz. Web-Inhaltsanalysen sind qualitativ konzipiert, wenn eine Untersuchung bestimmte Anbieter oder „CrossPlattform-Kommunikation" in den Blick nimmt. Online-Diskursanalysen und Video- \& Bildanalysen werden anhand verschiedener Paradigmen und in Bezug auf unterschiedliche Erkenntnishorizonte, welche dem Ursprung der Verfahren entsprechen, umgesetzt. Multimodale Analysen verbinden schließlich Ansätze 
der Cultural Studies, der Semiotik, der Linguistik und der Bildanalyse. So wird die Analyse multimedialer Social-Media-Inhalte möglich (Brügger, 2010; Fraas, Meier, \& Pentzold, 2013; Herring, 2010; Kress, 2009; O’Halloran, \& Smith, 2011; Pauwels, 2012; Welker, \& Wünsch 2010; Zeller, 2017a, S. 397; Zeller, 2017b, S. 395). ${ }^{29}$

Neben ihrer Auseinandersetzung mit den verschiedenen Varianten von SocialMedia-Analysen thematisiert Zeller die Erweiterung des empirischen Forschungsprozesses der Sozialwissenschaften aufgrund von Big Data und sozialen Medien beziehungsweise der neuen Art von Daten, die diese markieren. Da die Begriffe „Big Data“ und ,soziale Medien“ oft synonym gebraucht werden, ist ein Bezug zu Big Data für Social-Media-Analysen und deren theoretische Betrachtung relevant. Den Grund hierfür sieht Zeller in der hohen Quantität der Daten, die über soziale Medien generiert und auch ,abfragbar" gemacht werden (Zeller, 2017a, S. 397; Zeller, 2017b, S. 389). Big-Data-Forschung umreißt Zeller stark vereinfacht als

[. . . ] Forschung mit sehr großen Datensätzen, zu deren Erfassung und Bearbeitung technische Hilfsmittel (d. h. Computerprogramme und/oder Großrechnerkapazitäten) unabdingbar sind (Zeller, 2017a, S. 397).

Wie zuvor in dieser Arbeit schon dargelegt, weist auch Zeller darauf hin, dass sich der Begriff „Big Data“ in den letzten Jahren fast inflationär verbreitet hat, aber kontrovers diskutiert wird. Es fänden sich Beschreibungen, die Big Data ,das Öl der Informatik“ nennen, als allgemeinen Wettbewerbsvorteil von Unternehmen begreifen oder aber das Potential zuschreiben, als Ersatz für eine wissenschaftliche Forschung zu fungieren (Zeller, 2017a, S. 397 f.; Zeller, 2017b, S. 389). Den letzten Aspekt illustriert Zeller mit Worten von Chris Anderson (2008), der sich, wie auch Kitchin (2017), allerdings schon früher, mit der ,These vom Ende der Theorie“" aufgrund von Big Data beschäftigt hat:

${ }^{29}$ Sowohl der technologische Fortschritt als auch die Interdisziplinarität der Methode bedingen es, dass neben den von Zeller thematisierten und zum Zeitpunkt der Verfassung dieser Forschungsarbeit gängigen Varianten der Social-Media-Analyse weitere Verfahren entwickelt und diskutiert werden. Hinzu kommt, dass es sich bei Social-Media-Analysen um ein aktuelles Forschungsthema handelt; zu diesem wurden in den letzten Jahren interdisziplinär und weltweit unzählige Beiträge veröffentlicht. Innerhalb dieser Arbeit können aus forschungsstrategischen und -ökonomischen Gründen längst nicht alle Ansätze vorgestellt werden. Einige innovative, auch spezifische, oft aber noch nicht gänzlich etablierte Varianten der SocialMedia-Analysen und spezielle Tools werden in den Sammelbänden Social Media Research Methods von Sloan und Quan-Haase (2017) und Digital Methods for Social Science. An Interdisciplinary Guide to Research Innovation von Snee, Hine, Morey, Roberts und Watson (2016) vorgestellt; ein Blick in diese ergänzt die Arbeiten von Zeller. 
Forget taxonomy, ontology, and psychology. Who knows why people do what they do? The point is they do it, and we can track and measure it with unprecedented fidelity. With enough data, the numbers speak for themselves. [...] There's no reason to cling to our old ways. It's time to ask: What can science learn from Google? (Anderson, 2008).

Als ,weniger utopisch und verklärend positiv“, sondern vermehrt kritisch begreift Zeller den Big Data Diskurs der universitären Forschung, insbesondere den der Geistes- und Sozialwissenschaften (Zeller, 2017a, S. 398; Zeller, 2017b, S. 389). ${ }^{30}$ In Bezug auf Big Data merken Terry Flew, Christina Spurgeon, Anna Daniels und Adam Swift (2012) an:

It is important to remember that data refers not only to numeric and statistical records, but to any form of information represented in a digitised format including text, audio, photographic, and video files. The application of computational processes and techniques to mine, categorise, filter, amplify and transform this data into discernable trends, patterns and accurate and meaningful summaries generates information (Flew, Spurgeon, Daniels, \& Swift, 2012, S. 160, Hervorheb. i. O.).

Klaus Jensen (2014) spezifiziert dies, indem er erklärt, dass Daten durchweg entweder gesammelt oder produziert werden:

Data are either found or made (Jensen, 2014, S. 229).

Im Hinblick auf digitale Kommunikation und damit auch in Bezug auf Big Data sowie Social Media handelt es sich laut Jensen sogar um

[... ] a new type and scale of data [.. ]: big data or metadata that indicate who did what, with which information, together with whom, when, for how long and in which sequences and networks (Jensen, 2014, S. 229, Hervorheb. i. O.).

Diese neuen Daten der digitalen Kommunikation sind, so stellt Zeller mit Jensen fest, als Hybrid anzusehen, da sie gesammelt und produziert werden. Zum einen würden die Daten durch die Nutzung sozialer Medien generiert, könnten also online ,gefunden“ und gesammelt werden; zum anderen würden die Daten hergestellt, da für ihre Extraktion entsprechende Algorithmen programmiert, also erst „gemacht“ werden müssen. Diese „Daten-Hybridisierung“ beeinflusse auch den etablierten Prozess der empirischer Sozialforschung; dessen Erweiterung im

\footnotetext{
${ }^{30}$ Eine kritische Diskussion des Begriffs „Big Data“ haben bereits in 2012, wie in der Einleitung dieses Kapitels teilweise schon beschrieben, Boyd und Crawford (2012) sowie Manovich (2012) vorgelegt.
} 
Zuge von Social-Media-Analysen hat Zeller graphisch dargestellt (Jensen, 2014; Zeller, 2017a, S. 398 f.; Zeller, 2017b, 389 f.).

Abbildung 3.4 zeigt links den traditionellen sozialwissenschaftlichen Forschungsprozess, auf dessen Phasen im Rahmen dieser Arbeit nicht näher eingegangen wird. ${ }^{31}$ Rechts ist dessen aufgrund sozialer Medien notwendige Erweiterung abgebildet; diese fügt sich zwischen die Phasen der Datenerhebung und der Datenanalyse ein beziehungsweise erweitert den Bereich der Datenerhebung (Zeller, 2017a, S. 399; Zeller, 2017b, S. 389 f.).

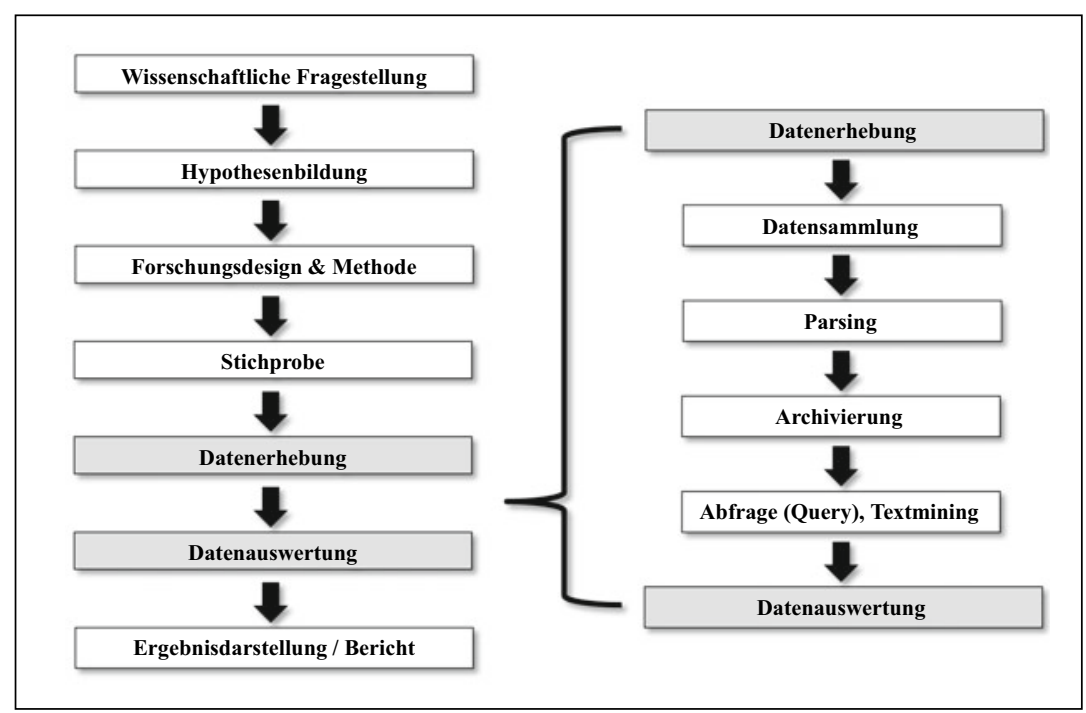

Abbildung 3.4 Forschungsprozess - Erweiterung bei Social-Media-Analysen (Zeller, 2017a, S. 399)

Gemäß Howard Welser, Marc Smith, Danyel Fisher und Eric Gleave (2008) besteht die Phase der Datenerhebung aus insgesamt vier Schritten, um die der Forschungsprozess bei Social-Media-Analysen aufgrund der neuen Art von Daten, die diese untersuchen, erweitert wird: (1) Datensammlung, (2) Datenerschließung und Datenaufbereitung, (3) Datenarchivierung, (4) Erstellung von Abfragen. (1)

${ }^{31} \mathrm{Da}$ dieser Forschungsprozess von kommunikationswissenschaftlicher Hand gezeichnet wurde, mutet er, zumindest auf den ersten Blick, als auf quantitatives Vorgehen ausgerichtet an. Mit entsprechenden Methodenkenntnissen ist eine Adaption für qualitative Verfahren möglich. 
Für die Datensammlung (Data Collection) werden bei Social-Media-Analysen computergestützte Verfahren genutzt; neben Application Programming Interfaces (APIs) kommen Web Crawler und Scraper zum Einsatz. APIs umfassen verschiedene Instrumente und Algorithmen, über die auf die Rohdaten der Betreiber sozialer Medien zugegriffen wird, um diese abzurufen. Web Crawler und auch Scraper laden Inhalte aus sozialen Medien systematisch herunter; entweder in Bezug auf vorab festgesetzte Themen oder im Hinblick auf Plattformen. (2) Während der Phase der Datenerschließung und -aufbereitung (Parsing) wird die oft große Menge gesammelter Rohdaten für die Analyse aufbereitet. In der Regel enthält das gesammelte Datenkorpus auch Rohdaten, die hinsichtlich der Fragestellung nicht benötigt werden; daher wird dieser systematisch durchgegangen und aussortiert, zum Beispiel von Spam befreit. Im Zuge der Datenaufbereitung werden die in Bezug auf die Fragestellung relevanten Rohdaten aus dem Datenkorpus extrahiert und in ein passendes Datenformat umgewandelt. (3 \& 4) Die Phasen der Datenarchivierung (Data Storing) und der Erstellung von Abfragen (Queries) fasst Zeller zusammen, da sie einer engen Abstimmung aufeinander bedürfen. Im Zuge der Datenarchivierung werden die Daten so gespeichert, dass sie bei Bedarf für Abfragen zur Verfügung stehen (Welser, Smith, Fisher, \& Gleave, 2008; Zeller, 2017a, S. 399 ff.; Zeller, 2017b, S. 390 f.; Zeller, 2014).

Zeller weist abschließend darauf hin, dass von der neuen Art der Daten, die im Rahmen von Social-Media-Analysen untersucht werden, nicht nur die Phase der Datenerhebung beeinflusst wird, sondern ebenso die daran anschließende Phase der Datenanalyse und auch der interpretative Umgang mit diesen Daten und den Analyseergebnissen. Bei dem Einsatz von Social-Media-Analysen in der Absicht, soziale Interaktionen in der sozialen Welt zu verstehen, gelte es, die Daten sozialer Medien in ihrem sozialen Kontext zu betrachten und zu kontextualisieren (Welser et al., 2008; Zeller, 2017a, S. 399 ff.; Zeller, 2017b, S. 386, S. 390 f.):

Linking social media data and other data sources can be regarded as a challenging and, at the same time, redeeming feature. It is challenging given the complexity of the data available online, and the research design needed for a sound research project analyzing multiple data formats and/or sources. The redeeming feature can be seen, for example, in the availability of vast quantities of different kinds of social media data, which help to contextualize findings and can potentially provide a work-around strategy to the anonymity challenge of online data. Social scientists are primarily interested in understanding social interaction embedded in our social world or context. This means we need to be able to contextualize data with the respective social world (usually demographic and other information). This is difficult - if not almost impossible given that anyone can pretend to be anybody on social media. Linking social media data and other data sources can help by enriching the results, and providing additional information (Zeller, 2017b, S. 386). 
Bei Social-Media-Analysen handelt es sich also um eine Methode, die unterschiedlich angewendet werden kann: Neben nicht-reaktiven sind reaktive Verfahren umsetzbar, neben quantitativen können qualitative Untersuchungen durchgeführt werden, neben einer automatisierten ist eine manuelle Umsetzung realisierbar. Sowohl an der Praxis von Social-Media-Analysen als auch an der Forschung mit der Methode und über sie sind mehrere Fachrichtungen beteiligt; dies wird im weiteren Verlauf dieser Arbeit noch deutlicher. Somit handelt es sich, wie zu Beginn schon erwähnt und nun mit Zeller dargelegt, um eine heterogene, mitunter auch diffuse Methode, die von Seiten der Sozialwissenschaften der Online-Forschung zugerechnet wird. Die Heterogenität und Diffusität der Methode gründet jedoch nicht nur in der großen Vielfalt der praktischen Umsetzungsmöglichkeiten von Social-Media-Analysen und der Beteiligung mehrerer unterschiedlicher Disziplinen, sondern ebenso in der Vielzahl möglicher Einsatzgebiete.

\subsection{Social-Media-Analysen aus weiteren Blickrichtungen}

Zeller (2017a, 2017b) hat, wie zuvor gezeigt, aus sozialwissenschaftlicher Perspektive einen ausführlichen und zur Zeit der Anfertigung dieser Arbeit aktuellen Überblick über Social-Media-Analysen vorgelegt. Im Hinblick auf die noch folgenden Ergebnisse der empirischen Untersuchung bildet vor allem dieser das theoretische Fundament der Methodik von Social-Media-Analysen in dieser Studie. Die Beschäftigung mit sozialen Medien und deren Analyse ist derzeit allerdings en vogue, weswegen eine Recherche nach Literatur, die deren Methodik und Methodologie behandelt, heute schon unzählige Ergebnisse hervorbringt und künftig wohl noch umfänglicher ausfallen wird. Einen umfassenden Literaturüberblick kann diese Forschungsarbeit nicht geben; lediglich ein Einblick ist möglich, basierend auf einer immer auch subjektiven, aber dem Fokus dieser Arbeit entsprechenden Selektion. Wegen ihrer Aktualität und insbesondere auch ihres sozialwissenschaftlichen Blicks wurden die Beiträge Zellers in den Vordergrund gerückt. Es soll jedoch nicht versäumt werden, auf weitere ausgewählte Arbeiten, die diese ergänzen, hinzuweisen; daher werden nun Social-Media-Analysen aus weiteren Blickrichtungen thematisiert.

Linda Lai und Wai Ming To (2015) unternehmen den Versuch, Social-MediaAnalysen als Methode anhand der Grounded Theory Methodologie zu bestimmen. Sie erkennen dabei eine Methode mit „hybridem Charakter“, die über eine quantitative Untersuchung „qualitative Inhalte“ sozialer Medien, wie die Eindrücke, Meinungen und Gefühle deren Nutzer, analysiert. Ihre Arbeit verstehen Lai und To selbst vor allem als einen Beitrag für Organisationen und deren E-Commerce-Aktivitäten: 
Nevertheless, social media content is widely accessible, up-to-date, and available in electronic format. Therefore, a systematic approach is necessary, as it helps electronic commerce researchers, organizations, and governments understand the commonality in various online text data that appear in social media. Using the information obtained from social media, researchers can gain valuable insights into the beliefs, values, attitudes, and perceptions of social media users with regard to the utility of usergenerated content and trust formation [. . . ]. Consequently, such information can help marketers monitor the perceptions of people regarding social networks and aid organizations in strategic planning. To address the gap between the availability of user-generated raw text and the contextual information of aggregated data, the present study introduces a grounded theory approach [. . . ] to analyze social media content to identify the underlying factor structure of the collected information and to interpret the identified structure in relation to the study objective (Lai, \& To, 2015, S. 138 f.).

Im Ergebnis unterscheidet sich das von Lai und To herausgearbeitete Bild von Social-Media-Analysen beziehungsweise des Forschungsprozesses der Methode kaum von der Darstellung Zellers; lediglich die Herangehensweise an die Methode über die Grounded Theory Methodologie ist neu. Eine Ergänzung zu Zeller besteht trotzdem darin, dass Lai und To die auch für diese Arbeit bedeutsame Relevanz von Social-Media-Analysen für Organisationen, wie Unternehmen, zeigen:

The decision making of managers is crucial to the survival of an organization, including government departments and business enterprises. With regard to the decision support process, the application of the proposed methodology [. . . ] highlights two areas of discussion, namely, social media content analysis as an information management tool and social media as a platform for building trust and information exchange (Lai, \& To, 2015, S. 148).

Lai und To verstehen in ihrer Betrachtung von Social-Media-Analysen die Methode als Werkzeug der Entscheidungsfindung, das dem Management von Organisationen zur Verfügung steht. Über sie sei es möglich, den Kunden ,zuzuhören'; dies, ohne dabei in deren Denkprozesse einzugreifen, wie es oftmals bei herkömmlichen Techniken der Marktforschung geschieht. Social-Media-Analysen beschreiben Lai und To damit als ,natürliche Beobachtungen'. Hinzu komme, dass soziale Medien Plattformen sind, auf denen ein Informationsaustausch erfolgt; dieser sei vor allem dadurch gekennzeichnet, dass er auf gegenseitigem Vertrauen der Nutzer beruht. Dies mache die Inhalte sozialer Medien bedeutsam für Managemententscheidungen (Lai, \& To, 2015, S. 148).

Neben den Kommunikations- und Wirtschaftswissenschaften, wie anhand der Beiträge von Zeller sowie Lai und To gezeigt, setzt sich vor allem die Informatik mit Social-Media-Analysen als Methode auseinander. Social-Media-Analysen 
werden mittlerweile, wie zuvor beschrieben, als Methode der Computational Social Sciences oder Digital Humanities gehandelt. Daher gibt diese Arbeit auch der Informatik einen gewissen Raum; allerdings wird nicht versucht SocialMedia-Analysen aus deren Blickwinkel darzustellen. Letzteres ist bereits an anderen Stellen mit entsprechendem fachlichem Hintergrund geschehen und wird dort vermutlich auch fortgeführt werden. Im Hinblick auf die Kooperation mit den Sozialwissenschaften bezüglich der Methode der Social-Media-Analysen hat die Informatik in den letzten Jahren einige Arbeiten veröffentlicht, von denen eine Auswahl nun noch gestreift wird. ${ }^{32}$

In ihrem Artikel The Power of Social Media Analytics (2016) legen aus Sicht der Informatik Weiguo Fan und Michael Gordon (2014) das Potential von Social-Media-Analysen dar. Anhand der Explosion sozialer Medien erkennen sie das Erfordernis von Social-Media-Analysen. Daher erklären sie den hinter der Methode liegenden Prozess und auch dessen Phasen, beschreiben die gebräuchlichsten Techniken und diskutieren, wie Unternehmen von der Methode profitieren können. Am Forschungsprozesses, den Fan und Gordon zeigen, wird für Sozialwissenschaftler die Notwendigkeit einer Kooperation von Informatik und Sozialwissenschaften ersichtlich. Im Gegensatz zu dem in dieser Arbeit nach Zeller bereits näher beschriebenen Prozess umfasst der von Fan und Gordon dargelegte insgesamt drei Phasen: (1) Capture, (2) Understand, (3) Present. In der Beschreibung der Phasen wird deutlich, dass nur wenig sozialwissenschaftliche Methodenkompetenz eingeflossen sein kann und Social-Media-Analysen als Methode der Informatik dargelegt werden; dies geschieht jedoch auf eine Art, die Social-Media-Analysen Nicht-Informatikern und Unternehmen näher bringen soll. Sechs Techniken nennen Fan und Gordon für die erste Phase: (1) Opinion Mining, (2) Sentiment Analysis, (3) Topic Modeling, (4) Social Network Analysis, (5) Trend Analysis, (6) Visual Analytics. Es sind zahlreiche Anwendungsfelder, die Fan und Gordon für Social-Media-Analysen in Unternehmen sehen; aus diesem Grund empfehlen sie die Methode für eine „Lebenszyklusanalyse“ von Produkten und Dienstleistungen:

\footnotetext{
${ }^{32}$ Es gibt unzählige weitere Beiträge der Informatik über Social-Media-Analysen. Siehe beispielsweise: Alhajj, \& Rokne, 2018; Atzmueller, Chin, Janssen, Schweizer, \& Trattner, 2016; Fu, 2014; Gaber, Cocea, Wiratunga, \& Goker, 2015; Goldbeck, 2013; Kawash, 2014; Kaya, Kawash, Khoury, \& Day, 2018; Kazienko, \& Chawla, 2015; Liu, 2015; Maynard, Roberts, Greenwood, Rout, \& Bontcheva, 2017; Meiselwitz, 2014, 2015, 2016, 2017a, 2017b; Missaoui, Abdessalem, \& Latapy, 2017; Moens, Li, \& Chua, 2014; Russel, 2013; Zafarani, Abbasi, \& Liu, 2014.
} 
Social media has changed our conversations about products and services but not the business activities underlying them. A life cycle analysis perspective considers the life of a product (or service) from its design through its disposal, as well as support activities that take place in parallel with these activities. [... ] one that is quite typical suffices for our purposes, having these four stages: design-development; production; utilization; and disposal [... ]. Social media is most relevant to the design-development and utilization stages, both of which we emphasize in our discussion. In addition, we comment as well on how social media analytics help firms gather competitive intelligence, i. e., help firms understand more completely their business environment, suppliers, and competitors (Fan, \& Gordon, 2014, S. 13 f.).

Genauer diskutieren Fan und Gordon die folgenden fünf Einsatzgebiete: (1) Product Design Development, (2) Product Production, (3) Product Utilization, (4) Product Disposal, (5) Competitive Intelligence. Die Methode halten sie vor allem dafür geeignet, Fragen des Marketings in Bezug auf die Nutzung sozialer Medien zu beantworten und hierzu Messungen durchzuführen. Trotz einiger Herausforderungen sprechen Fan und Gordon Social-Media-Analysen das Potential zu, die Geschäftswelt zu wandeln:

At the same time, social media are transforming the very nature of business. [. . . ] As we have suggested, social media are now supporting the "co-creation" of products, with consumers working online with companies' product designers [. . . ]. As these new commercial frontiers open, technical challenges loom. The extraordinary volume of "big data" will challenge social media analytics [. . . ]. Language issues add further complications as businesses begin to monitor and analyze social media conversations around the world. These challenges may swell as social media analytics begin to incorporate user-based location data facilitated by mobile technology and pressures rise to process and respond to social messages in real-time (Fan, \& Gordon, 2014, S. 21).

Einen Beitrag der Informatik, der sich explizit an die sozialwissenschaftliche Forschung richtet, haben Bogdan Batrinca und Philip Treleaven (2015) vorgelegt; auch sie gehen nicht nur auf die Potentiale von Social-Media-Analysen ein, sondern behandeln ebenso deren Herausforderungen, wozu sie auch die nur bedingte Aktualität ihres Beitrags wegen des rasanten technologischen Fortschritts zählen:

This paper is written for (social science) researchers seeking to analyze the wealth of social media now available. It presents a comprehensive review of software tools for social networking media, wikis, really simple syndication feeds, blogs, newsgroups, chat and news feeds. For completeness, it also includes introductions to social media scraping, storage, data cleaning and sentiment analysis (Batrinca, \& Treleaven, 2015, S. 89). 
Aus Sicht der Informatik betrachten Batrinca und Treleaven die Methodologie von und Kritik an Social-Media-Analysen. Sie erachten eine experimentelle Rechenumgebung als notwendig für die Methode. Insgesamt beschreiben sie Social-Media-Analysen zwar als eine Methode der Computational Social Sciences, sprechen sich aber auch für deren ,quantitative Ausschließlichkeit' aus, indem sie Social-Media-Analysen ausschließlich als für quantitative Messungen geeignet halten:

Social media is especially important for research into computational social science that investigates questions [. . . ] using quantitative techniques (e. g., computational statistics, machine learning and complexity) and so-called big data for data mining and simulation modeling [... ] (Batrinca, \& Treleaven, 2015, S. 89).

Die Position ,quantitativer Ausschließlichkeit` vertreten Bogdan und Treleaven, obwohl sie die Eignung von Social-Media-Analysen zur Erzeugung von Verständnis sehen:

Social media data is clearly the largest, richest and most dynamic evidence base of human behavior, bringing new opportunities to understand individuals, groups and society. Innovative scientists and industry professionals are increasingly finding novel ways of automatically collecting, combining and analyzing this wealth of data (Batrinca, \& Treleaven, 2015, S. 90).

Anhand des Beitrags von Batrinca und Treleaven wird deutlich, dass die Informatik nur anhand von Quantitäten beziehungsweise quantitativen Messungen Verständnis erzeugt und damit mitunter auf eine andere Weise als die Sozialwissenschaften, der neben den quantitativen Methoden bekanntlich auch qualitative zur Verfügung stehen. Doch nicht nur im Hinblick auf ihre grundlegende Methodik können sich die Informatik und die Sozialwissenschaften voneinander unterscheiden, auch ihre Beschäftigung mit Social-Media-Analysen kann sehr verschieden sein; dies macht eine gegenseitige Ergänzung umso fruchtbarer. Batrinca und Treleaven behandeln neben unterschiedlichen Social- Media-Daten verschiedene Social-Media-Provider, worauf diese Arbeit jedoch ebenso wenig eingehen wird wie auf die von ihnen erläuterten Varianten des Textaufbereitung, -markierung und -speicherung oder die zahlreichen Techniken, Tools und Plattformen für Social-Media-Analysen, die die Informatik für die Untersuchung sozialer Medien und deren Inhalte bereithält. Zusammenfassend erkennen Batrinca und Treleaven in der einfachen Verfügbarkeit sozialer Medien die Explosion deren Analysen begründet. Ihr größtes Bedenken besteht darin, dass Unternehmen den Zugriff auf Daten einschränken könnten, um diese zu monetarisieren. Damit 
bliebe der Forschung der Datenzugang verwehrt und Social-Media-Analysen würden, wohl ausschließlich in ihrer quantitativen Variante, zur ,exklusiven Domäne von Großkonzernen“:

Perhaps, the biggest concern is that companies are increasingly restricting access to their data to monetize their content. It is important that researchers have access to computational environments and especially 'big' social media data for experimentation. Otherwise, computational social science could become the exclusive domain of major companies, government agencies and a privileged set of academic researchers presiding over private data from which they produce papers that cannot be critiqued or replicated. Arguably what is required are public-domain computational environments and data facilities for quantitative social science, which can be accessed by researchers via a cloud-based facility (Batrinca, \& Treleaven, 2015, S. 115).

Anhand des Beitrags von Rui Gaspar, Cláudia Pedro, Panos Panagiotopoulos und Beate Seibt (2016) wird hier der Bogen von der Informatik zurück zur Psychologie und über diese zu den Sozialwissenschaften und in die Nähe Zellers gespannt; dies geschieht in Bezug auf ein zentrales Element vieler Social-Media-Analysen, die Stimmungsanalysen oder auch Sentiment-Analysen. Anders als die Informatik verstehen Gaspar et al. diese nicht als quantitative Untersuchungen, sondern beschreiben deren qualitative Variante. Ihr Artikel Beyond Positive or Negative: Qualitative Sentiment Analysis of Social Media Reactions to Unexpected Stressful Events (2016) behandelt eine Beispielstudie. ${ }^{33} 34$ Zwar sehen Gaspar et al. Anwendungsfälle für quantitative Stimmungsanalysen, merken aber an, dass diese gegebenenfalls nicht ausreichen und qualitativ zu ergänzen sind:

There are however valid reasons to believe that computer-based sentiment analysis techniques may not be adequate to assess social media reactions on their own; and that another complementary layer of human-based assessment should be put forward. More than triggering positive or negative affective reactions, potentially stressful events can be perceived both at the individual and social levels as posing threats or challenges to cope with, depending on the resources available and the demands posed by them [...]. Moreover, these perceptions may change over time, along the chain of events that might occur - the hazard sequence [. . . ]. This type of assessment requires a deeper understanding of people's affective expressions on social media and the context in which people express sentiment and other cognitive and behavioural manifestations while events unfold (Gaspar et al., 2016, S. 180).

${ }^{33}$ Diese Forschungsarbeit kann aus forschungsökonomischen wie -strategischen Gründen nicht auf die von Gaspar et al. umgesetzte qualitative Sentiment-Analyse und die dieser zugrunde liegende Theorie und deren Methodik wie Ergebnisse eingehen.

${ }^{34}$ Sentiment-Analysen behandelt aus dem Blickwinkel der Digital Humanities beispielsweise auch Hai-Jew (2017b, 2017c). Aus forschungsstrategischen wie -ökonomischen Gründen wird diese Diskussion in dieser Studie nicht vorgestellt. 
Gaspar et al. stellen fest, dass die qualitative Untersuchung von Beiträgen ein tieferes Verständnis des emotionalen Zustands der Nutzer sozialer Medien erzeugen kann als es Bewertungen wie ,positiv“, „,negativ“, „,neutral“" und ,ambivalent“ vermögen. Wegen der A-priori-Annahmen, die quantitativen Stimmungsanalysen zugrunde liegen, seien diese eingeschränkt:

1) [S] entiment is a one-dimensional concept characterised by valence (positive, negative, neutral, ambivalent), 2) circumscribed to a small set of emotions (e. g. fear, anger, surprise) and 3) expressed with no visible/explicit goal or function, or even "irrationally" (Gaspar et al., 2016, S. 180).

Oftmals sei dies der Fall bei unerwarteten und potentiell belastenden sozialen Krisen, die als eine Verschärfung der negativen, zu neutralisierenden Stimmung dargestellt werden. Gaspar et al. betonen, dass die Bedeutung der Äußerungen von Menschen so vielfältig wie individuell und daher qualitative Stimmungsanalysen unerlässlich sind:

Therefore, the valence of people's expressions during unexpected and potentially stressful events may only be the visible part of the "iceberg". [. . . ] In other words, people do not simply panic in response to an emergency but cope in diverse collective and individual ways [. . . ]. It is therefore important to consider the richness of social media expressions through qualitative analysis that takes into account all dimensions of valence (positive, negative, neutral, ambivalent) with respect to the underlying functions or goals that affective expressions may serve (Gaspar et al., 2016, S. 181).

Infolge ihrer Beispielstudie kommen Gaspar et al. zu dem Schluss, dass qualitative, manuell durchgeführte Stimmungsanalysen komplex und zeitaufwändig sind, außerdem nicht derart skalierbar und verständlich wie quantitative. Trotzdem, so betonen Gaspar et al., erzeugen qualitative Stimmungsanalysen, welche den Kontext der untersuchten Inhalte einbeziehen, tiefere und umfassendere Erkenntnisse im Hinblick auf die von Menschen formulierten Botschaften. Allerdings würden Social-Media-Analysen auch das Risiko mit sich bringen, dass anhand der Methode beziehungsweise der gesamten Big-Data-Forschung die sozialwissenschaftliche Trennung quantitativer und qualitativer Methoden eine neue Legitimierung erfährt. Mit Nachdruck sprechen sich Gaspar et al. gegen eine solche aus. Stattdessen propagieren sie neue methodische Wege über die Kombination beider Varianten sowie über eine Verbindung von Mensch und Maschine durch die Computational Social Sciences: 
As Boyd and Crawford (2012; p. 667) referred: “(...) working with Big Data is still subjective, and what it quantifies does not necessarily have a closer claim on objective truth - particularly when considering messages from social media sites. But there remains a mistaken belief that qualitative researchers are in the business of interpreting stories and quantitative researchers are in the business of producing facts. In this way, Big Data risks re-inscribing established divisions in the long running debates about scientific method and the legitimacy of social science and humanistic inquiry". [... ] Still, both approaches are limited in this regard, thus making salient the need to implement new methods to increase coding validity, for example increasing the number of independent coders and including a human validation step in computer based analysis. Moreover, attempts should be made at implementing mixed methods approaches and mixed computer-human based approaches, which may overcome limitations that each face separately. In addition to limitations, there is also value to qualitative sentiment analysis approaches. Arguably, sentiment analysis and monitoring of stressful and unexpected events, can support effective communication strategies and interventions (Gaspar et al., 2016, S. 188 f.).

\subsection{Einsatzgebiete von Social-Media-Analysen: Marktforschung im Web 2.0}

Social monitoring tools are search engines that track the content generated by Internet users. They allow companies to track customer conversations about brands, products, and services, measure the reputation of the brand, check the response of consumers after launching an advertising campaign, analyze brand-related sentiment, identify opinion leaders (influencers), develop online strategies, and resolve crisis situations (Soler-Labajos, \& Jiménez-Zarco, 2017, S. 50 f.).

Weiter führen Soler-Labajos und Jiménez-Zarco (2017) acht potentielle Gründe für die Anwendung von Social Monitoring Tools in Unternehmen an: (1) Markenwahrnehmung der Nutzer erfassen; (2) Community der Nutzer kennenlernen; (3) Möglichkeiten zur Verbesserung des Nutzererlebnisses entdecken; (4) Erkenntnisse zur Optimierung von Produkten und Kundenservice gewinnen sowie mit Verbrauchern kommunizieren; (5) Positionierung der Marke im digitalen Umfeld überprüfen; (6) Kampagnenwirkung evaluieren; (7) Zeiträume vergleichen und Veränderungen analysieren; (8) Return on Investment (ROI) messen. Bald in ähnliche, bald in weitere Richtungen tendieren die neun möglichen Einsatzgebiete von Social Media Monitoring Tools in Unternehmen, die Harriet Kasper, Moritz Dausinger, Holger Klett und Thomas Renner (2010) nennen: (1) Reputationsmanagement; (2) Event Detection, Issues- und Krisenmanagement; (3) Risikomanagement; (4) Wettbewerbsbeobachtung; (5) Trend- und Marktanalysen; (6) Kampagnen-Monitoring; (7) Meinungsführeridentifikation; (8) Customer 
Relationship Management; (9) Produkt- und Innovationsmanagement (Kasper, Dausinger, Klett, \& Renner, 2010, S. 8 ff.; Soler-Labajos, \& Jiménez-Zarco, 2017, S. 51$){ }^{35} 36$

Neben den Anwendungsfällen, die Soler-Labajos und Jiménez-Zarco sowie Kasper et al. zusammengefasst haben, wurden im Verlauf dieses Kapitels bereits zahlreiche weitere Einsatzgebiete von Social-Media-Analysen angeführt. Die diversen Zwecke, für die die Methode in Unternehmen eingesetzt wird, verdeutlichen deren Vielfalt. Die Absicht dieses Abschnitts, der die Einsatzgebiete von Social-Media-Analysen zusammenfasst und dabei die Marktforschung im Web 2.0 exemplarisch herausgreift, besteht jedoch nicht darin, erneut die Heterogenität und Diffusität der Methode darzulegen; vielmehr wird mit der Marktforschung ein Anwendungsfall der Verfahren herausgegriffen und als Einsatzgebiete von Social-Media-Analysen: Marktforschung im Web 2.0 beschrieben. Nicht nur in Bezug auf die organisationale Wissensgenese kommt der Marktforschung Bedeutung zu; neben der universitären beziehungsweise wissenschaftlichen Forschung beschäftigt sich vor allem die kommerzielle Marktforschung mit der Methode der Social-Media-Analysen. In der Entwicklung und Erprobung neuer Erhebungsmethoden scheint die Marktforschung der Wissenschaft teilweise sogar voraus zu sein und zeigt ihr neue Möglichkeiten (Zeller, 2017a, S. 402). Zeller (2017) ist es allerdings ein Anliegen, hierzu zu ergänzen,

\footnotetext{
${ }^{35}$ Im Praxishandbuch Ask, Measure, Learn. Using Social Media Analytics to Understand and Influence Customer Behavior (2014) betrachten außerdem Finger und Dutta Social-MediaAnalysen anhand diverser Einsatzgebiete oder auch Unternehmensfunktionen: Marketing, Sales, PR, Customer Care, Social CRM: Market Research, Gamig the System, Predictions.

${ }^{36}$ Einen Einblick in Issues Monitoring und Management und auch Krisen- und Risikomanagement bieten Wiedemann und Ries (2014). Der Zusammenhang von Corporate Foresight und Issues Management steht im Fokus bei Kuhn, Ruff und Splittgerber (2014). Medienanalysen thematisieren Blumhoff und Seiffert (2014). Aßmann und Pleil (2014) stellen allgemeine Grundlagen und Zielsetzungen von Social Media Monitoring mit einem Schwerpunkt auf die Unternehmenskommunikation vor und geben einen Einblick in Erfolgsmessung, Benchmarking, Kundeninsights und Kundenservice, Markt- und Themenanalysen, Issues und Agenda Setting und auch Reputation. Harder (2014) wirft den Blick auf Dienstleistungen für die Unternehmenskommunikation und damit in Richtung der Agenturen, die Social Media Monitoring im Auftrag von Unternehmen und damit auch für diese durchführen. Schwarz und Löffelholz (2014) setzen sich mit Krisenkommunikation auseinander und beleuchten Risikomanagement und Prävention.
} 
[. . . ] dass es insbesondere im Bereich universitärer Forschung liegt, neuen Trends und vor allem empirischen Messmethoden die notwendige kritische Aufmerksamkeit bezüglich deren Validität zukommen zu lassen. Da die universitäre Forschung eben nicht demselben extremen Zeitdruck unterliegt wie die kommerzielle Forschung, und zudem unabhängig ist, zählt es auch zu ihren Pflichten, die notwendige Zeit zu investieren, um die Wahrung wissenschaftlicher Standards zu garantieren. Hierzu zählen auch Fragestellungen rechtlich-ethischer Natur, welche insbesondere in der empirischen Forschung über und mit soziale(n) Medien eine gesonderte Rolle spielen (Zeller, 2017a, S. 402).

Es ist anzumerken, dass aufgrund der regen Beschäftigung der Marktforschung mit Social-Media-Analysen zahlreiche Literaturbeiträge über die Methode weniger der wissenschaftlichen als einer wirtschaftlichen Feder entstammen; , die Marktforschung' nimmt oft in Gestalt von Dienstleistungsunternehmen oder Agenturen für kommerzielle Marktforschung am Diskurs teil. Zudem ist zu erwähnen, dass die für Marktforschung zuständige Einheit von Unternehmen eine Durchführung von Social-Media-Analysen in der Regel entweder bei einer Agentur beauftragt oder die Methode über die Nutzung von Tools praktiziert oder aber die Umsetzung von Social-Media-Analysen manuell erfolgt. Dienstleister wiederum führen Social-Media-Analysen entweder manuell oder mit Tools oder aber anhand selbst entwickelter beziehungsweise erworbener Software durch. Im Internet stehen sowohl kostenlose als auch kostenpflichtige Tools bereit. ${ }^{37} 38$

Grundsätzlich wird die Marktforschung eher der Wirtschaftswissenschaft und innerhalb dieser dem Marketing zugerechnet als der Sozialforschung. Doch bedient sie sich meist deren Methoden, oftmals in ,pragmatischer Abwandlung', was ihr zuweilen das Etikett „quick and dirty“ einbringt. Da es für den weiteren Verlauf dieses Abschnitts wesentlich ist und sich zudem in den Ergebnissen der empirischen Untersuchung, auf denen diese Studie beruht, wiederfindet, ist ein wiederholt innerhalb der Literatur auftauchendes, methodisches Missverständnis ‘ anzusprechen, dem wohl manche Wirtschaftsforschung und damit auch Marktforschung zu unterliegen scheint. Soler-Labajos und Jiménez-Zarco, beide Wirtschaftswissenschaftler, beschreiben Social-Media-Analysen wie folgt:

\footnotetext{
${ }^{37}$ In ihrem weiteren Verlauf verwendet diese Arbeit die Bezeichnungen „Dienstleister“ und „Agenturen“ synonym.

${ }^{38}$ Einige Agenturen, die die Methode in Gänze oder Tools oder auch Software für deren Durchführung kommerziell vertreiben, haben den Prozess der von ihnen praktizierten SocialMedia-Analysen sowie die dabei zum Einsatz gebrachten Verfahren dargelegt. Siehe zum Beispiel: Böck, Köbler, Anderl, \& Le, 2017; BVDW, 2011; Hill, Dean, \& Murphy, 2014; IBM, 2010. Eine nicht mehr aktuelle Marktstudie über kommerzielle Anbieter von SocialMedia-Analysen und auch verfügbare Tools findet sich bei Kasper, Dausinger, Kett und Renner (2011).
} 
Social media analysis measures, studies, and interprets information that is generated in social media by quantifying data extraction and identifying the feeling showed by the users when they talk (Soler-Labajos, \& Jiménez-Zarco, 2017, S. 51).

Weiter konkretisieren sie die Methode, indem sie quantitative und qualitative Verfahren unterscheiden:

- Quantitative metrics measure numerical terms (e. g., number of visits to the company web page of the company, number of Twitter followers, number of views of YouTube videos).

- Qualitative metrics measure concepts related to perception, feeling, and the level of engagement of the users with the brand (e. g., the character of the mentions the brand receives, as positive, negative, or neutral) (Soler-Labajos, \& Jiménez-Zarco, S. 2017, S. 51, Hervorheb. i. O.).

In diesen beiden Zitaten von Soler-Labajos und Jiménez-Zarco offenbart sich das eben angesprochene ,methodische Missverständnis', dem etliche Praktiker von Social-Media-Analysen zu unterliegen scheinen: Wie zuvor in dieser Arbeit, im Zusammenhang der wissenssoziologischen Konzeption von Max Weber schon dargestellt, unterscheidet die empirische Sozialforschung quantitative von qualitativen Methoden und charakterisiert Erstere als erklärende, Letztere hingegen als verstehende Verfahren. Mit qualitativen, verstehenden Methoden nehmen Sozialwissenschaftler jedoch keine Messungen vor, sondern leisten Interpretationen und Deutungen. Es kann hier zwar nicht vollkommen ausgeschlossen werden, dass die Wirtschaftswissenschaft ein im Grundsatz anderes Methodenrepertoire nutzt als die empirische Sozialforschung, angezweifelt wird dies jedoch erheblich. Daher gilt es, das ,methodische Missverständnis', welches offenbar manchen wirtschaftswissenschaftlichen Arbeiten zugrunde liegt, in der Beschäftigung mit Social-Media-Analysen im Kontext der Marktforschung zu bedenken. ${ }^{39}$

\footnotetext{
${ }^{39}$ Unternehmen nutzen Social-Media-Analysen, wie erwähnt, nicht nur zum Zweck der Marktforschung, sondern insbesondere auch im Marketing, um beispielsweise die Wirkung von Kampagnen zu evaluieren oder für die Ermittlung von Influencern. Daher liegen inzwischen viele Arbeiten vor, die die Methode mit Fokus Marketing thematisieren. Zwar wird darin die Methode verschieden bezeichnet, der Titel enthält aber oft die Bestandteile „Analytics“ oder „Monitoring“ und ist in der Regel als Social-Media-Analyse, zumeist mit einer quantitativen Schwerpunktsetzung, auszumachen. Aus forschungsstrategischen und auch -ökonomischen Gründen verzichtet diese Studie darauf, Social-Media-Analysen aus der Perspektive des Marketings sowie als dessen Methode gesondert vorzustellen; die sozialwissenschaftliche Darlegung der theoretischen Aspekte von Social-Media-Analysen gilt genauso für das Marketing, das in seiner Praxis eigene Schwerpunktsetzungen vornimmt. Als spezielle
} 
Patrick Brauckmann und Madeleine Baldauf (2014) diskutieren Web Monitoring als ein "Instrument des Vertriebs“ und damit zugleich als eines der Marktforschung, das zum Zweck der Wissensgenese über die Kunden und Wettbewerber eingesetzt wird:

Längst sind alle Branchen vom Verteilungswettbewerb in den Verdrängungswettbewerb eingetreten. Im Rahmen eines solchen Wettbewerbs gewinnt das Wissen über Kunden und Konkurrenten wieder an Bedeutung. Dabei stellt sich die Frage, wie aus vertrieblicher Sicht eines Unternehmens Kundenmeinungen über ein Web-Monitoring aufgenommen und in ein Unternehmen integriert werden können. Beeinflusst davon sind der Produkt-Entwicklungs-Prozess ebenso wie Vertrieb und Marketing. Auch entstehen Veränderungen und Effekte auf die vertriebliche Organisation in Unternehmen, die wiederum Anpassungen im Rahmen eines Web-Monitorings notwendig machen (Brauckmann, \& Baldauf, 2014, S. 469).

Ähnlich wie Brauckmann und Baldauf rechtfertigen Soler-Labajos und JiménezZarco den Einsatz von Social Monitoring Tools in Unternehmen, gehen dabei aber noch einen Schritt weiter: Sie erklären, dass über Social-Media-Analysen erworbenes Wissen zur unternehmerischen Entscheidungsfindung beiträgt, vielmehr Unternehmen überhaupt erst zum Handeln befähigt:

We define information as a set of processed data that has significance (relevance, purpose, and context) and is useful for making decisions because it reduces uncertainty [...]. However, it is knowledge that allows us to act; knowledge integrates experience, values, and know-how, then uses them as the framework for the incorporation of new information and experiences. To gain knowledge and know whether the actions developed in social media are properly routed toward the goals we intend to achieve, it is necessary to measure and analyze information. Measurement allows us to know the results of the activities developed in social media, to check whether our objectives have been achieved and, above all, to value the performance of our strategies and the impact they have on the economic benefits to our companies (Soler-Labajos, \& Jiménez-Zarco, 2017, S. 51).

Damit sind die Beweggründe der Marktforschung, Web Monitoring oder auch Social Monitoring anzuwenden, als durchaus gewichtig für Unternehmen einzustufen.

Marketing-Literatur zu Social-Media-Analysen können folgenden Beispiele genannt werden: Gonçalves, 2017; Grabs, \& Bannour, 2014; Hemann, \& Burbary, 2013; Lamberton, \& Stephen, 2016; Sterne, 2011; Tuten, \& Solomon, 2018; Walsh, Hass, \& Kilian, 2011; Werner, 2013. Des Weiteren behandeln und systematisieren Misirlis und Vlachopoulou (2018) 52 Artikel, die von 2010 bis 2016 in Peer Review Journals erschienen oder auf internationalen Konferenzen vorgestellt worden sind. 
In der gesichteten Literatur ist keine Rede davon, die Marktforschung durch Social-Media-Analysen zu ersetzen; es geht stets um eine Ergänzung des Methodenrepertoires der Marktforschung und um die Kombination etablierter Methoden mit Social-Media-Analysen. Dirk Steffen (2014) schlägt für Ansätze, die die Methoden der klassischen Marktforschung mit Social-Media-Analysen verbinden, die Bezeichnung ,hybride Verfahren“ vor und betont,

[... ] dass Social-Media-Methoden für einige Forschungsfragen ausreichende Qualität bereitstellen, bzw. eine erste schnelle Orientierung ermöglichen. Für Forschungsgebiete, die auf Hochrechenbarkeit auf eine Grundgesamtheit angewiesen sind und einen definierten Qualitätsstandard sicherstellen müssen, bleiben sie jedoch unterlegen (Steffen, 2014, S. 108).

Daher plädiert Steffen für Verfahrenskombinationen, die es erlauben, zugleich sowohl die Vorteile, wie Geschwindigkeit, Skalierbarkeit, Dialogfähigkeit et cetera zu nutzen als auch eine quantitative beziehungsweise statistische Validierung der Ergebnisse zu erzielen (Steffen, 2014, S. 108).

Des Weiteren ist festzustellen, dass die Marktforschung in den bisherigen Beispielen nicht von ,Social-Media-Analysen“ spricht; ob sie nun als eine Subdisziplin der Wirtschaftswissenschaft oder als organisationale Einheit eines Unternehmens auftritt, die oft der des Marketings angegliedert ist. Stattdessen wählt sie die Bezeichnung „Social Media Monitoring“" oder Variationen davon. Der Grund hierfür ist einerseits wohl in dem bereits dargelegten ,methodischen Missverständnis ' zu sehen, andererseits in der ,methodischen Vorliebe', die die Wirtschaftswissenschaft, das Marketing und die Marktforschung für quantitative Methoden hegen, wie in dieser Arbeit auch schon gezeigt. Zwar bevorzugt die Marktforschung Social Media Monitoring als Variante von Social-Media-Analysen, spricht dieser aber keine Ausschließlichkeit zu, sondern kennt zudem deren Variante des Social Media Listening. Zum Beispiel unterscheidet Steffen Social Media Monitoring Tools von Social Media Listening Tools, jedoch ohne dabei deren Unterschied zu spezifizieren. Auch Alex Gonçalves (2017) thematisiert Social Media Monitoring und Social Media Listening; Ersteres wird als Analytik dargelegt, Letzteres metaphorisch mit ,Zuhören' assoziiert. Allerdings fügt Gonçalves hinzu, dass beide Bezeichnungen teilweise auch synonym gebraucht werden (Gonçalves, 2017; Herrmann, \& Moeller, 2006, S. 114 f.; Steffen, 2014, S. 98):

Social media listening received this name because it relates to the analyst being able to "hear" what the market is saying about the brand via social media channels. Many marketers also refer to it as social media monitoring (Gonçalves, 2017, S. 59, Hervorheb. i. O.). 
Ferner werden Social Monitoring und Social Listening explizit von Tracy Tuten und Michael Solomon (2018) unterschieden und teilweise anhand einer weiteren Metapher definiert:

Social monitoring and listening go hand in hand. Social monitoring is the process of tracking mentions of specific word or phrases on social media sites. Monitoring enables companies to be notified when a social mention warrants a reaction. The monitoring process acts like a trigger. Social listening also identifies and collects information shared on social media sites, but for listening applications, the data collected is analysed for insights to inform strategic marketing decisions. Though both activities mine social data, monitoring is reactive when listening is proactive. Dan Neely, CEO of Networked Insights, described the difference like this "Monitoring sees trees; listening sees the forest" (Tuten, \& Solomon, 2018, S. 317). ${ }^{40}$

Sozialwissenschaftlich betrachtet handelt es sich bei Social Media Listening in gewisser Weise um die qualitative Variante von Social-Media-Analysen. Allerdings würde eine methodisch fundierte und reflektierte Sozialforschung qualitatives Vorgehen nicht als ,Zuhören“, abtun“ und herabwürdigen, wie dies bei Gonçalves geschieht. Im Gegensatz zu diesem erkennt nämlich zum Beispiel Kate Crawford (2009) durchaus Potential in der Metapher des ,Zuhörens“ und entwickelt

$[\ldots]$ the concept of listening as a metaphor for paying attention online. Pejorative terms such as 'lurking' have failed to capture much detail about the experience of presence online. Instead, much online media research has focused on 'having a voice', be it in blogs, wikis, social media, or discussion lists. The metaphor of listening can offer a productive way to analyse the forms of online engagement that have previously been overlooked, while also allowing a deeper consideration of the emerging disciplines of online attention (Crawford, 2009, S. 525).

Listening beschreibt Crawford als eine Metapher für die Teilnahme an Diskussionen und Debatten im Internet; es sei ein nützliches Mittel, um die Erfahrungen der Nutzer sozialer Medien festzuhalten. Organisationen beziehungsweise Unternehmen haben, so Crawford, längst die Vorteile eines ,Zuhörens ‘ in sozialen Medien erkannt. Mit Social Media Listening würden diese nun versuchen, eine engere Beziehung zu ihren Kunden aufzubauen, Informationen über Produkte zu erhalten und auch ihre eigene öffentliche Wahrnehmung zu verbessern:

\footnotetext{
${ }^{40}$ Mit beständigem Fokus auf das Einsatzgebiet Marketing, einschließlich spezieller Anmerkungen in Richtung Marktforschung, legen Tuten und Solomon (2018, S. 319 ff.) den Forschungsprozess von Social-Media-Analysen dar und thematisieren zudem unterschiedliche Verfahren der Methode.
} 
For companies, the value of listening could be considered in three ways: being seen to participate in a community and hearing people's opinions; utilizing a rapid and lowercost form of customer support (as compared to the telephone); and gaining a dispersed global awareness of how a brand is discussed and the patterns of consumer use and satisfaction (Crawford, 2009, S. 531 f.).

Jedoch ist nach Ansicht Crawfords weitere Forschung zu den „Listening Practices" in sozialen Medien vonnöten; diese könnte weitere Ansätze auftun, um die Nuancen der Vernetzung und Kommunikation, die Social Media bieten, noch besser zu verstehen. ${ }^{41}$

Social Media Monitoring deckt demgegenüber die vorrangig quantitativen Verfahren beziehungsweise Bestandteile von Social-Media-Analysen ab. Wohl wegen der generell eher quantitativen Ausrichtung der Marktforschung scheint diese mitunter nur wenig Bewusstsein für qualitative Methoden zu haben, zumindest in Bezug auf Social-Media-Analysen. Durch das Herabstufen von Listening gegenüber Monitoring wird letztlich auch dem qualitativen Vorgehen sein Potential abgesprochen. Außerdem erklärt die verstärkt quantitative Ausrichtung, welche die Marktforschung aufweist, nicht nur den Vorzug, den sie quantitativen Verfahren gibt, sondern auch, dass die Bezeichnung „Social Media Listening“ im Vergleich zu „Social Media Monitoring“, erheblich seltener in der Literatur und in Praxisberichten zu finden ist.

Wie schon angesprochen, ist das Potential qualitativer Varianten von SocialMedia-Analysen und vor allem von Methodenkombinationen längst erkannt worden. Dieser Befund spiegelt sich auch in den Stimmen der Marktforschung sowie in Arbeiten wider, die Social-Media-Analysen mit Bezug zu dieser behandeln; dies wird im Folgenden anhand einiger Beiträge veranschaulicht. ${ }^{42}$

\footnotetext{
${ }^{41}$ Aus forschungsökonomischen wie -strategischen Gründen kann diese Arbeit nicht näher auf die von Crawford (2009) entfaltete Metapher des ,Zuhörens' in sozialen Medien eingehen. Mittlerweile haben sich zum Beispiel auch Stewart und Arnold (2017) weiter mit Social Listening beschäftigt, dieses auf eine Literaturrecherche hin als ,,[...] an emerging type of listening and as a means of attaining interpersonal information and social intelligence that can empower relationships and influence the way we listen to and communicate with one another through increasingly popular mediated channels" (Stewart, \& Arnold, 2017, S. 1) definiert und außerdem als „Organizational Social Listening“ dargelegt.

${ }^{42}$ Lee (2017) systematisiert von Seiten des Informatik Social-Media-Analysen für Unternehmen anhand einer Typologie und der Darstellung ihres Prozesses sowie unterschiedlicher Verfahren. Auf diesen gut zugänglichen und aus der Sichtweise der Informatik wohl umfassenden Überblick wird im Rahmen dieser Arbeit wegen deren sozialwissenschaftlichen Fokus jedoch nicht eingegangen. Trotzdem ist zu erwähnen, dass auch Lee Marketing als primäres Einsatzgebiet von Social-Media-Analysen in Unternehmen nennt; der Aufbau von Social Media Intelligence sei das vorrangige Ziel des Einsatzes der Methode. Zwar betont Lee,
} 
Michael Schenk, Monika Taddicken und Martin Welker (2008) hinterfragen das Web 2.0 bezüglich der Chancen und Herausforderungen, die es für die Marktforschung und Sozialforschung bereithält, vor allem im Zusammenhang mit der Datenerhebung:

Insgesamt wird im Web 2.0 eine Fülle an Informationen über die Einstellungen, Meinungen, Vorlieben, Wünsche und Kritikpunkte der Web-2.0-Nutzer durch die Nutzer selbst generiert. Auch Produkte, Marken und Konsumerlebnisse sind oftmals Thema von Beiträgen in Blogs und Foren. Die Informationsfülle kann somit wertvolle Aspekte für die Forschung enthalten. Vereinzelt werden das Web 2.0 im Allgemeinen und Blogs im Speziellen entsprechend sogar als ,Goldgrube der Marktforschung ' bezeichnet (Schenk, Taddicken, \& Welker, 2008, S. 244).

Den Innovationsgrad des Web 2.0 erkennen Schenk et al., die Social-MediaAnalysen in erster Linie als eine explorative und damit qualitative Methode auffassen, in der neuen Offenheit der Nutzer von sozialen Medien; Vernetzung und Interaktivität seien deren zentrale Konstitutionsmerkmale. Entsprechend spannen sie einen Klassifikationsrahmen für Studien im Web 2.0 anhand einiger sozialwissenschaftlicher Schlüsselbegriffe und Forschungsbezüge auf, die den fünf zentralen Konzepten von Social Media entsprechen: (1) „Koorientierung“ und kollektive Intelligenz ${ }^{43}$, (2) Partizipation und Dynamisierung, (3) Interaktion beziehungsweise Kommunikation und Interaktivität, (4) Authentizität und virtuelle Gemeinschaft, (5) Selektion und Aggregation sowie Rekombination. Neue Möglichkeiten der Datenerhebung ergeben sich laut Schenk et al. vor allem durch das veränderte Nutzerverhalten und neuartige Anwendungen. Dank der „Koorientierung" des Web 2.0 (1) könne die Forschung individuelle Entscheidungen, die schließlich zu öffentlichen Gütern führen, verfolgen; dies sei im Hinblick auf die Simulation von Märkten relevant. Über Social-Media-Analysen kann damit gemäß Schenk et al. die Entstehung von Trends und Meinungen untersucht werden:

dass dafür alle Aktivitäten interdisziplinär gestaltet werden und Monitoring wie Listening umfassen müssen, doch zeigt die Darlegung des Prozesses und der Verfahren von SocialMedia-Analysen, dass die Methode wieder einzig quantitativ gedacht wird. Zurückzuführen ist dies wohl erneut auf die quantitative Ausrichtung von Informatik und Wirtschaftswissenschaft. ${ }^{43}$ Die das Web 2.0 auszeichnende „Koorientierung“ oder eben auch kollektive Intelligenz beruht auf der „Weisheit der Vielen“, die Surowiecki in seinem Werk The Wisdom of Crowds (2005) beschreibt. Hinter diesem Konzept steckt die systemtheoretisch begründete Annahme, dass eine Gruppe von Individuen gemeinsam ein Problem besser zu lösen vermag als dies ein einzelnes Individuum alleine kann. Die Kompetenz zur Lösung eines Problems wird zwar, so die Vorstellung, auf kollektiver Ebene erreicht, aber trotzdem durch das Handeln Einzelner, das sich begrenzt an anderen Individuen orientiert (Schenk et al., 2008, S. 247). Einen Überblick gibt zum Beispiel auch Send (2012). 
Aus Perspektive der Marktforschung können also insgesamt hilfreiche Erkenntnisse durch die sowohl quantitative als auch qualitative Analyse von Web-2.0-Anwendungen gewonnen werden, beispielsweise innerhalb der Trendforschung und für die Problemund Kaufentscheidungsanalyse. Auch wird ein effizientes regelmäßiges Monitoring als Frühwarnsystem oder für PR- und Marketing-Controlling-Zwecke ermöglicht, das ohne den Rückgriff auf das Web 2.0 nur mit höherem Ressourceneinsatz durchführbar wäre (Schenk et al., 2008, S. 256).

Weiterhin ermögliche die Interaktivität des Web 2.0 (3) den Einsatz dialogorientierter Instrumente. Diese würden ein besseres Verständnis der Zielgruppe generieren, anhand dessen beispielsweise die Marketingmaßnahmen optimiert werden könnten. Schenk et al. erkennen darin ferner neue Gestaltungsräume,

[. . . ] um leistungsfähige Wertschöpfungsmuster und flexiblere Kooperationsformen der Neuproduktentwicklung zu schaffen. Dieser Ansatz der, Community-based Innovation' kann sowohl in verschiedenen Prozessphasen verfolgt werden als auch mittels unterschiedlicher Rollenverständnisse bzw. -interpretationen, bis hin zum Web-2.0-Nutzer als ,Co-Produzent‘ [ . . ] (Schenk et al., 2008, S. 257).

Zudem profitiere die Forschung von der zunehmenden Vernetzung der Nutzer; dies sei sowohl in der Partizipation des Einzelnen (2) begründet als auch in der Authentizität seiner Äußerungen (4). Über bestimmte Anwendungen könnten Daten zur Analyse der Funktionsweise sozialer Netzwerke erhoben und ferner soziale Beziehungen der Nutzer analysiert werden. Dadurch werde es für die Markt- und Meinungsforschung möglich, Meinungsführer zu identifizieren; Schenk et al. verweisen auf virales Marketing und Schneeballeffekte. ${ }^{44}$ Das Zwischenfazit von Schenk et al. lautet:

\footnotetext{
${ }^{44}$ Der Begriff „Meinungsführer“ oder „Opinion Leader“ entstammt der Studie The People's Choice. How the Voter Makes Up his Mind in a Presidential Campaign (1944/1968) von Lazarsfeld, Berelson und Gaudet. Durch diese wurde erstmals erkannt, dass in sozialen Gruppen einige wenige Meinungsführer eine besonders aktivierende Wirkung auf andere Gruppenmitglieder haben. Meinungsführer sind dadurch gekennzeichnet, dass sie besser informiert und vernetzt sind als ihr soziales Umfeld; des Weiteren sind sie gut in ihre Gruppe integriert und im Umgang mit deren Normen vertraut. Aus diesen Gründen wird ihnen eine meinungsbeeinflussende oder auch -verstärkende Funktion zugesprochen. Meinungsführer stellen somit eine strategische Komponente bei der Bildung von Gruppenmeinungen und im Prozess sozialen Einflusses dar. Unternehmen sprechen die Meinungsführer ihrer eigenen Zielgruppe bewusst an. Im Zusammenhang mit sozialen Medien werden Meinungsführer heute meist „Influencer" genannt (Schenk et al., 2008, S. 257 f.). „Influencer“ ist eine Bezeichnung von Seiten des Marketings; siehe hierzu: Tuten, \& Solomon, 2018, S. 84 ff..
} 
Insgesamt ermöglicht das Social Web also neue Formen der Einblicke in die ,Black Boxes ' der Nutzer und Konsumenten, sodass für die Forschung neue Möglichkeiten gegeben sind, individuelle und soziale Prozesse zu verfolgen bzw. Kunden besser zu verstehen und so ihre Wünsche und Bedürfnisse besser befriedigen zu können (Schenk et al., 2008, S. 258).

Den angesprochenen Chancen, die Social-Media-Analysen der Marktforschung bieten, sehen Schenk et al. allerdings auch einige Herausforderungen gegenüberstehen, die den im Verlauf dieses Kapitels bereits thematisierten entsprechen: Die Auswertung der Informationen sei mit hohem Aufwand verbunden; in einem sensiblen Feld, wie es soziale Medien darstellen, würden ethische und rechtliche Grundsätze die Offenlegung der Forschungsaktivitäten erfordern; bei quantitativen Auswertungen könne es zu Fehlinterpretationen kommen; qualitative Auswertungen würden eines hohen Einsatzes von Ressourcen bedürfen; wegen der digitalen Spaltung und des damit einhergehenden Mangels an Repräsentativität seien die Ergebnisse nicht verallgemeinerbar; trotz der Authentizität der Inhalte könnten diese unwahr sein und seien nicht überprüfbar. Obwohl diese Einschränkungen bestehen und manche Fragen noch unbeantwortet sind, konstatieren Schenk et al. aber ein großes Potential, das Social-Media-Analysen in der Markt- und Sozialforschung zukommt; dieses sehen sie in den drei Grundprinzipien des Web 2.0, seiner Offenheit, Vernetzung und Interaktivität, begründet:

\begin{abstract}
Aus diesem Grund kann dem Research 2.0 abschließend ein großes Forschungspotenzial zugestanden werden. Dieses beruht (derzeit) vor allem auf der Verwendungsmöglichkeit für die explorative Forschung, weshalb Research 2.0 eine ideale Ergänzung zu anderen Markt- und Sozialforschungsergebnissen darstellt. Insbesondere bietet sich der qualitativen Online-Forschung die Chance, sich zu etablieren. Für die Zukunft dürfen wir deshalb (weiterhin) viele interessante Ergebnisse aus dem Research 2.0 erwarten (Schenk et al., 2008, S. 262).
\end{abstract}

Joachim Scholz (2008) beleuchtet das Verhältnis von klassischer Marktforschung zu Online-Forschung. Im Gegensatz zu Schenk et al. betrachtet er Social-MediaAnalysen aber als quantitative Verfahren, wie er sämtliche Online-Forschung als quantitativ begreift. Deren zögerlichen Einzug und zaghafte Etablierung in Deutschland, die sich verglichen mit den Zuwachsraten der Nutzer des Web 2.0 unterproportional entwickelt, sind auch heute, lange Zeit nach der Veröffentlichung des Beitrags von Scholz, noch zu verzeichnen. Bereits 2008 hat Scholz dieses Phänomen mit enormen Vorbehalten gegenüber dem neuen Instrument zur Datenerhebung, als das er Social-Media-Analysen ansieht, erklärt. Laut Scholz ist die deutsche empirische Sozialforschung stolz auf ihre wissenschaftliche Tradition; diese sei mit den Mängeln der Marktforschung im Web 2.0 nicht zu vereinen und dennoch werde sich Letztere etablieren: 
Und doch haben sich die Rahmenbedingungen geändert, und kaum ein Branchenkenner zweifelt mehr daran, dass das Internet künftig eine bedeutende Rolle in der Forschung spielen wird. [. . . ] Online-Forschung wird zudem auch bahnbrechende Veränderungen in den empirischen Methoden mit sich bringen. Denn bei aller berechtigten Kritik [. . . ]: Immer mehr setzt sich die Erkenntnis durch, dass mittels OnlineForschung Forschungsfragen beantwortet werden können, die mittels keiner anderen Erhebungsmethode realisiert werden können (Scholz, 2008, S. 230 f.).

Veränderung in der Datenerfassung bewertet auch Scholz als den größten Wandel. Das Web 2.0 biete spezielle Darstellungstechniken und Interaktionsmöglichkeiten mit den Nutzern. Allerdings fehle es noch an Standards und verlässlichen Verfahren. Auch die Bedeutung der visuellen Kommunikation wird gemäß Scholz zunehmen:

Ein Bild sagt mehr als tausend Worte. Denn die Lebenswelten der Zielgruppen unterscheiden sich deutlich von den Vorstellungen der Produktentscheider in den Unternehmen. Ein Blick auf Konsum und Umfeld via Handyfoto dürfte unpräzise Milieudefinitionen mindestens ergänzen, wenn nicht sogar ersetzen. Der Versuch, mit Zielgruppentypologien mehr über den, unbekannten' Konsumenten zu erfahren, glückt nur zum Teil. Direktes Feedback in Form von Bildern wird erheblich mehr Aufklärung leisten (Scholz, 2008, S. 235).

Zwar befänden sich die Erhebungs- und Auswertungstechniken für Bildmaterial noch in der Entwicklung, doch würde dessen Bedeutung im Hinblick auf Erkenntnisse über die Lebenswelten der Konsumenten steigen. Auch multimodale Optionen seien attraktiv, vor allem im Hinblick auf Online-Befragungen; Scholz nimmt an, dass dank dieser die Teilnahmebereitschaft, die derzeit auf ein bedenklich niedriges Niveau gesunken ist und die Studienrepräsentativität gefährdet, steigen wird. Insgesamt sieht Scholz aber nicht nur Chancen, sondern auch zahlreiche Herausforderungen, denen eine Marktforschung, die online agieren möchte, gegenübersteht. Im Forschungsalltag seien die Argumente für die Online-Forschung noch nicht angekommen. Es mangle bislang an gangbaren Wegen, adaptierten Methoden und statistischen Auswertungsverfahren; oft fehle es den Forschungsinstituten an Informatikkenntnissen. Noch etliche Hürden seien in Richtung virtueller Forschungswelten zu überwinden. Zwar wurden, so merkt Scholz an, Blogs und Communities bereits als vielversprechende Quellen für die Marktforschung erkannt, doch in diesen werden auch etliche spezifische Themen diskutiert: 
Viele dieser Diskussionen haben in der Vergangenheit in abgeschlossenen, nicht zugänglichen Zirkeln stattgefunden, jetzt sind sie in der Regel jedem zugänglich. $\mathrm{Ob}$ daraus forscherisch valide und reliable Erkenntnisse gewonnen werden können, ist noch offen. [. . . ] Entweder, weil die Zuordnung der Daten zu einer bestimmten Zielgruppe nicht gelingt, oder, weil entscheidende Informationslücken eine Interpretation unmöglich machen (Scholz, 2008, S. 239).

Meinungsführer betrachtet Scholz in Bezug auf die Daten als Problem; diese würden die Diskussion dominieren und Einfluss auf Konsumenten ausüben, wobei deren Meinung nicht deckungsgleich mit der des Verbraucherdurchschnitts sei. Trotz der Hürden, die es noch zu nehmen gilt, ist letztlich aber auch Scholz vom Erfolg der Online-Forschung überzeugt:

Die Online-Forschung wird weiter wachsen. Für die Forscher ergeben sich neue, spannende Möglichkeiten - und neue Herausforderungen. Die Zahl der offenen Fragen und Problemstellungen sind bei allem Erfolg noch mannigfaltig (Scholz, 2008, S. 241).

Unter der Parole „Listening Is the New Asking“ thematisieren Holger Lütters und Marc Egger (2013) Social-Media-Analysen im Kontext der Marktforschung und beschreiben die Methode als einen nicht-reaktiven, eher qualitativen Ansatz, der Auswirkungen auf klassische reaktive Erhebungsverfahren hat. Ihre These ist, dass die Marktforschung in sozialen Medien ,zuhören' kann, anstatt klassische Befragungen durchzuführen:

Die in den sozialen Netzwerken entstehenden Datensammlungen sind prädestiniert für die Integration in den Marktforschungsprozess [. . . ]. Mit der gewachsenen Menge an Nutzerbeiträgen und der gestiegenen Bedeutung im Prozess der Kaufentscheidung eröffnet sich für die Marktforschung eine neue Grundlage zur Generierung von Erkenntnissen. Die Analyse dieser riesigen Datenmengen stellt eine Herausforderung für Marketing und Marktforschung dar und wird derzeit auch im Kontext des Stichwortes Big Data diskutiert (Lütters, \& Egger, 2013, S. 34).

Lütters und Egger beleuchten verschiedene Ansätze der Social-Media-Forschung in der Praxis und legen den gesamten Prozess von Social-Media-Analysen als ein vierstufiges, iteratives Modell dar. Dieses beinhalte die Schritte der Datensammlung, deren Analyse, der Darstellung und der Interpretation. Während die ersten drei Schritte, so Lütters und Egger, von Maschinen übernommen werden, obliegt der letztgenannte dem Menschen: 
Die vor wenigen Jahren noch unvorstellbaren Möglichkeiten der automatisierten Datenerhebung und der neuen Methoden der Analyse sozialer Netzwerke zum Zwecke der Marktforschung erscheinen faszinierend. Der Marktforscher erhofft sich durch der [sic] Social Media-Forschung die primär maschinelle Verarbeitung auf allen Prozessstufen. Zuweilen wird dabei vergessen, dass diese Schritte weiterhin nicht allein maschinell erfolgen können und nicht nur die Interpretation der aufbereiteten Daten letztendlich dem Menschen obliegt (Lütters, \& Egger, 2013, S. 40).

Schwierigkeiten werden im Zusammenhang mit der Frage nach relevanten Quellen, die einer Social-Media-Analyse zugrunde liegen, sowie in Bezug auf die Repräsentativität hinsichtlich der Grundgesamtheit durchschnittlicher Konsumenten gesehen. Obwohl die Rede ist vom „Potential, die Marktforschung zu revolutionieren“, sehen Lütters und Egger noch erhebliche Herausforderungen hinsichtlich der technischen Machbarkeit und juristischen Bewertung. Ihre Zukunftsprognose für die Marktforschung lautet:

Die Zukunft der Marktforschung wird in der Anwendung eine Kombination aus technischem Know-how gepaart mit einem fundierten Verständnis für die Mechanismen der Analyse erforderlich machen. Diese Grundvoraussetzungen dürfen vom klassischen Marktforscher heute noch nicht erwartet werden. Der Marktforscher sollte sich aber für die Zukunft darauf einstellen, die Social Media-Analyse als einen selbstverständlichen Baustein in einem integrierten Methodenkanon verschiedener qualitativer und quantitativer sowie reaktiver und nicht-reaktiver Methoden zu betrachten (Lütters, \& Egger, 2013, S. 40 f.).

Anhand eines Fallbeispiels beschreiben Matthias Schultze und Annika Postler (2008), wie ein Unternehmen seinen Kunden mit Online-Trend-Monitoring ,zuhört'. Ziel sei es Stimmungen zu identifizieren und Frühwarnsignale zu erkennen. $\mathrm{Zu}$ diesen Zwecken würden folgende Fragestellungen untersucht: (1) Wie viel kommuniziert wird, (2) was diskutiert wird, (3) wer in welchem Kontext teilnimmt, (4) wo der Austausch stattfindet. Ursprünglich habe das Unternehmen das Web 2.0 als neues Kommunikationsmedium in Richtung der Kunden wahrgenommen, inzwischen habe es sich des Weiteren zu einem wichtigen Analysemedium entwickelt. Entsprechend erkennen Schultze und Postler im Web 2.0 auch Erweiterungsmöglichkeiten für die klassische Marktforschung:

Denn wer als Unternehmen besonders innovativ und kundennah agieren will, muss das ,Ohr am Verbraucher' haben und alle Möglichkeiten nutzen, um neue Impulse aufzunehmen. Und was eignet sich - neben der klassischen Marktforschung - dabei besser als das Internet mit seinen vielfältigen Möglichkeiten, in die Belange der Konsumenten hineinzuschauen und ihre Bedürfnisse und Forderungen, aber auch Kritik und Verbesserungswünsche aufzunehmen (Schultze, \& Postler, 2008, S. 371). 
Im Gegensatz zum hohen Aufwand klassischer Marktforschung seien von Social Media Monitoring schnelle und kostengünstige Informationen erwartet worden. Es handle sich dabei allerdings nicht um ein Werkzeug, das fertige Ergebnisse liefert; vielmehr bestehe dieses aus unterschiedlichen Verfahren, die das Internet nach festgelegten Begriffen und Themen durchforsten. In Abhängigkeit von Aufgabenstellung und Analysebedarf werde entschieden, welche Module zu verwenden sind. Zum Einsatz komme es in Bezug auf unterschiedliche Fragstellungen und Themen, wobei es zwei Vorgehensweisen gebe: Einen Online-Trend-Radar sowie Ad-Hoc-Untersuchungen. Mittlerweile begreift das Unternehmen SocialMedia-Analysen, so Schultze und Postler, als zentralen „Baustein im Reporting“; sowohl die Ergebnisse der Webstatistik als auch die der Marktforschung würden durch die Methode ergänzt werden. Solange Social-Media-Analysen aber nicht repräsentativ seien, sei nicht damit zu rechnen, dass die kostenintensive Marktforschung durch diese ersetzt wird. Einen eindeutigen Vorteil der Methode erkennen Schultze und Postler darin, dass diese nicht-reaktiv ist, , beobachtend ' agiert und auf diese Weise eine Ergebnisverzerrung ausschließt.

Nichtsdestoweniger stecken die Verfahren der Online-Analyse (vor allem im Vergleich zur ,klassischen “ Marktforschung) noch in den Kinderschuhen. Wir sehen hier einen großen Entwicklungsbedarf. Valide Ergebnisse werden sich erst im Zusammenspiel von Wissenschaft und Forschung, Unternehmen und Internet-Marken wie Google \& Co. erzielen lassen (Schultze, \& Postler, 2008, S. 381).

Hartmut Scheffler (2014) diskutiert des Weiteren, ob es sich bei Social-MediaAnalysen um einen Hype oder Zukunftstrend handelt. Er merkt an, dass in sozialen Medien die unterschiedlichsten Daten entstehen, die, da von Individuen produziert, per se für die Markt- und Sozialforschung vom Interesse sind. Schefflers Ergebnis lautet:

Social Media ist aus dem täglichen Leben der allergrößten Anzahl der Menschen nicht mehr wegzudenken und diesen Menschen wird in Zukunft eine Vielzahl neuer Angebote gemacht, eine Vielzahl neuer Möglichkeiten eröffnet. Social Media ist kein Hype, sondern relevante Realität und als solche Gegenstand der Forschung (Scheffler, 2014, S. 17).

Social Media beziehungsweise die darin schlummernden „Datenschätze“ seien von der Marktforschung längst als Forschungsgegenstand erkannt worden. Ferner haben sich, so Scheffler weiter, bereits zwei verschiedene Richtungen etabliert: Zum einen die passive Nutzung und Auswertung existierender Daten, zum anderen die aktive Nutzung zur Erzeugung von Daten. Die Marktforschung hat, 
so erklärt Scheffler, ihre zentralen Themenfelder daraufhin beleuchtet, inwieweit soziale Medien bei seit jeher bestehenden und immer wiederkehrenden Fragen neue Lösungen und Methoden bereithalten oder auch neue Fragen aufwerfen und zugleich ihre Beantwortung ermöglichen. Nach den Themenfeldern der Marktforschung gegliedert, gibt Scheffler hierzu einige Beispiele:

Social Media als Gegenstand der Forschung wird die notwendigen Prozesse von Unternehmen und Marken im Hinblick auf die Herausforderungen der Digitalisierung begleiten. Social Media wird Gegenstand der Forschung bleiben und noch verstärkt werden, weil es sich bei Social Media um ein gesellschaftliches Phänomen mit wachsender Bedeutung handelt, weil Social Media im Tagesablauf einer zunehmenden großen Anzahl von Menschen nicht mehr wegzudenken ist und weil Social Media folglich relevant für Kommunikation, für Informationssuche, für Einstellungsbildung und -änderung bis hin zum Verhalten [. . . ] ist (Scheffler, 2014, S. 18f.).

Zentrale Themen in Bezug auf soziale Medien als Gegenstand der Marktforschung sind für Scheffler methodologische Fragen mit dem Ziel eines optimierten Methodenkanons und die Beantwortung sich wiederholender Fragestellungen. Insgesamt macht Scheffler drei Komponenten aus, die die Anforderungen an soziale Medien als Gegenstand der Marktforschung antreiben: (1) Entwicklung und Planung großer Anbieter, (2) daran angepasste, an Volumen zunehmende Fragestellungen von Unternehmensseite, (3) Möglichkeiten durch das mobile Internet. Ebenso wie soziale Medien neue Daten und Erkenntnismöglichkeiten bereithalten, bieten sie laut Scheffler ein neues Spektrum an Instrumenten: (A) Aktive, (B) passive, (C) interaktive Methoden. (B) Mit passiven Verfahren erfolge die ,Beobachtung ‘ und Messung von Verhaltensdaten; dazu werden Social-Media-Analysen in Form von Social Media Monitoring und die Erfassung von Klickverhalten gezählt. (A) Aktive Ansätze seien Befragungen, die sowohl unabhängig vom Verhalten in sozialen Medien stattfinden als auch unmittelbar daran anschließen könnten. (C) Interaktive Methoden sind für Scheffler Crowdsourcing und Communities. Scheffler folgert: 
Es gibt eine Vielzahl an Instrumenten und Tools, die stärker als bisher in Richtung der Chancen, aber auch Grenzen des Einsatzes und in Richtung der Transparenz der Methodik und damit der Eignung für ausgewählte Fragestellungen dokumentiert werden müssen. Social Media schafft völlig neue Erkenntnismöglichkeiten, hat aber auch eindeutig systemimmanente Grenzen, von denen die zwei wichtigsten die Unklarheit der Grundgesamtheit, auf die hin verallgemeinert werden kann, zum einen und die Gefahr von gefakten Informationen zum zweiten sind. Ein nächster Schritt muss deshalb die Verknüpfung von Social Media Research, Social Media Monitoring mit klassischen Instrumenten sein. So können z. B. Informationen aus dem Suchverhalten, aus Beobachtung/Webtracking, aus Social Media Monitoring/Inhaltsanalyse kombiniert werden mit (repräsentativen) Befragungen. Die zweite Aufgabe der Marktforschung ist die einer stärkeren Systematisierung in Form einer Strukturierung der Forschungsgegenstände, Vereinheitlichung der Begriffe, Strukturierung der Methoden, vor allem Vereinheitlichung der Metriken und nicht zu vergessen, der Festlegung von methodenangepassten Ethik- und Qualitätskriterien (Scheffler, 2014, S. 23).

Ein Spannungsverhältnis zwischen Marketing und Marktforschung erkennt Jillian Ney (2016) in Bezug auf Social-Media-Analysen und fragt:

Are Social Analysis Tools Holding Back Social Media Intelligence as a Market Research Method (Ney, 2016, S. 1)?

Ursprung der Frage sei die Feststellung, dass „Social Media Intelligence“, die laut Ney in der Industrie gängige Bezeichnung für Social-Media-Forschung, enormen Problemen gegenüberstehe; beispielhaft nennt sie die Unfähigkeit, bestehende Forschungsfragen zu beantworten, und das Fehlen von Modellen zur Analyse. Ursache dieser Probleme und dafür, dass sich Social-Media-Analysen noch nicht als Methode der Marktforschung etabliert hätten, seien die für die quantitativen Messungen des Marketings entwickelten Tools:

Such challenges are further compounded by the social tool domination of the industry and their limited analytical functionality for research purposes. The very tools that researchers must use to analyse large volumes of social media conversations are stalling the growth of social media intelligence as a market research method. The industry has been plagued by a flood of underwhelming insights reports because of the promise from social tools to 'find insight in the noise', and it could be argued that this marketing promise is degrading the perception of what an insight should be. The tools have been developed for marketers as end users, not researchers, and the focus on quantitative measures and fuzzy metrics are problematic for a researcher looking to generate deep customer insight. Currently social tools are not attempting to change their measurement approach and it is left to the Researcher to overcome the limited functionality to effectively answer traditional market research questions (Ney, 2016, S. 1). 
Mit den vorhandenen Tools sei es der Marktforschung fast nicht möglich, die geforderte Analysetiefe zu erreichen. Wenn exakte Forschungsziele gesetzt, ein maßgeschneiderter Rahmen für Untersuchungen entwickelt und mehrere Tools verwendet würden, könnten Social-Media-Analysen jedoch neue, bisher eventuell übersehene Fragen beantworten. Ney nennt vier Herausforderungen, denen Social-Media-Forschung in Organisationen gegenübersteht: (1) Focus on Marketing Measurement, (2) Social Tool Domination, (3) Lack of Measurement Metrics, (4) Human Behaviour.

(1) Organisationen würden Social-Media-Analysen für Marketingzwecke einsetzen und dadurch das Potential der Social-Media-Forschung verkennen:

There are indications that social media intelligence could be something more powerful to organisations if the focus of analysis is extended beyond marketing measurement, and that the need for this new analysis approach may still be unknown to the market. The idea that the market does not know what they need, are not aware of what is available, or do not understand how a given technology or service can benefit them [... ] is plausible given that social media intelligence is a relatively new concept. To date, Social analysis tools have been developed to assist in analysing large volumes of unstructured data in respect of the tasks or 'jobs to be done' [ . . ] of marketers. Meaning that the analysis of social media is both led and constrained by social analysis tools on the market. The focus of marketers on measuring brand content, engagement and performance and the need for social analysis tools to provide the means to achieve this may be perpetuating the problem of 'underwhelming' insights and slowing the use of social media intelligence in market research (Ney, 2016, S. 3).

(2) Das Marketing habe sich ausschließlich auf Tools konzentriert und die wichtigste Phase von Social-Media-Analysen, die Interpretation der Daten, übergangen; daher seien auch die Ansprüche des Marketings an Tools falsch. Ney betont, dass Tools keine Erkenntnisse generieren; um an verwertbare Erkenntnisse zu gelangen, müssten Daten interpretiert werden:

The most important stage of data analysis, the interpretation stage, has largely been excluded from discussion and has seen a change in what an insight is perceived to be. The control of social media intelligence initiatives by marketing functions, the need to focus measurement on marketing output and active engagement, and the emphasis of social tools on pursuing marketers are their target customer has led to professionals interpreting the data who are inexperienced in developing customer or market insight. This inexperience has directly impacted on the type of insight generated, and the quality of this insight because their inexperience fails to recognise that the marketing claims of social analysis tools are false. Social analysis tools do not provide insight - the user must interpret the data from the tool to generate actionable business intelligence (Ney, 2016, S. 3). 
Die vom Marketing praktizierte lösungsorientierte Sichtweise auf die Methode und die Fokussierung auf Metriken und quantitative Messungen mit Tools behindert, so Ney, die Etablierung von Social-Media-Analysen in der Marktforschung. Letztere müsse zur Beantwortung ihrer Forschungsfragen in andere Analysetiefen vorstoßen können.

(3) Ney bewertet Social-Media-Forschung in Bezug auf die fehlenden Messgrößen in zweifacher Hinsicht als herausfordernd: Zum einen mangle es an etablierten Praktiken und auch an Literatur bezüglich der Entwicklung der Verfahren; zum anderen könnten Forscher ihre Fragen nicht leiten, da SocialMedia-Analysen passive und nicht-reaktive Verfahren sind. Die Entwicklung von Methoden und Metriken sei nötig:

$[\ldots]$ the same data can be analysed both qualitative and quantitatively $[\ldots]$. The challenge becomes the development of research methods and metrics which can extract value by combining these two perspectives (Ney, 2016, S. 4).

Hinzu kommt des Weiteren, so Ney, das menschliche Verhalten (4), das die Inhalte von sozialen Medien und auch die Kommunikation in diesen bestimmt:

The unstructured nature of social media data is driven human participation, thus rendering content and communication subject to the psychology of human behaviour. [... ] In a business sense, social data is the artefact of communications that are making or breaking brands. Experience has become the new cultural capital, with an increase in individuals 'lifecasting' [...] insights about their daily life. [... ] As a market research method, social media intelligence can offer a new, effective approach to the study of human behaviour when the associated challenges of analysis have been overcome (Ney, 2016, S. 5).

Wenn die angeführten Herausforderungen der organisationalen Social-MediaForschung überwunden sind, stehe der Marktforschung in Gestalt von SocialMedia-Analysen eine innovative Methode zur Untersuchung menschlichen Verhaltens zur Verfügung. Ney befindet die Social-Media-Forschung als geeignet für organisationale Marktforschung: 
Social media intelligence should focus on assisting organisations to identify their strengths, weaknesses, opportunities and threats as well as provide insight to strategically plan for future decisions. To successfully capture the market research industry, social analysis tools must consider the 'jobs to be done' [... of the researcher and not shoehorn these tasks into the tool configuration for marketing measurement. Although social media intelligence can offer a new avenue for market research, it's widespread adoption in business is being held back because the social analysis tools are focused on the measurement of marketing performance (Ney, 2016, S. 9).

Ney fordert von Unternehmen, zu erkennen, dass Tools keine Erkenntnisse generieren; weder würden sie strategische Einblicke geben noch Fragen beantworten. Zwar hätten Tools, da sie den Datenzugriff erleichtern und als kostengünstig empfunden werden, die Zugangsbarrieren zur Marktforschung reduziert, doch dies würde sich auf die Qualität der Erkenntnisse und deren Umsetzbarkeit in Unternehmensentscheidungen auswirken.

Social analysis tools are controlling what 'insight' is perceived to be, and a wave of inexperienced professionals are perpetuating 'underwhelming' insights reports that are holding back the credibility of social media intelligence as a market research method (Ney, 2016, S. 10).

Somit kommt Ney zu dem Schluss, dass Marktforschung durch Tools nicht zu ersetzen ist. Tools der Zukunft sollten, so Ney, darauf abzielen, Marktforschung zu unterstützen, ein besseres Verständnis des Kundenverhaltens zu generieren, die Bedürfnisse und auch Befürchtungen der Kunden zu verstehen und die Markenmessung zu optimieren. Ferner fordert Ney, dass die „Black Boxed Algorithms“ der Tools transparenter werden; nur dann seien deren Einschränkungen und Schwachstellen zu erkennen.

Der vorliegende Abschnitt hat Einsatzgebiete von Social-Media-Analysen, allen voran die Marktforschung im Web 2.0 thematisiert. Dabei wurden Listening und Monitoring als zwei Varianten von Social-Media-Analysen kontrastiert und deren Potentiale wie Herausforderungen im Kontext der Marktforschung dargelegt. Mit der Unterscheidung von passiven, aktiven und interaktiven Verfahren wurde zudem eine Option gezeigt, die in der Sozialforschung etablierte Klassifizierung von Methoden in quantitative und in qualitative Ansätze aufzubrechen. Darüber werden Social-Media-Analysen verstanden als eine „Methodeninnovation“, die neu klassifizierbar ist. Die Klassifizierung geschieht in Abhängigkeit 
vom Verhalten des Forschers, das aktiv oder passiv sein kann, und vom Einbezug der Nutzer sozialer Medien, der ausbleiben kann oder erfolgen kann und die Methode dann interaktiv werden lässt. Passive Methoden lassen sich des Weiteren in Monitoring und Listening untergliedern. In Bezug auf die Datenauswertung umschreibt Monitoring ein ,Beobachten 'von Social Media, das zumeist automatisiert erfolgt und quantitative Ergebnisse hervorbringt; Listening meint demgegenüber ein ,Zuhören' in sozialen Medien, das in Bezug auf den Einbezug des Kontexts der Inhalte oft manuell umgesetzt wird und zu diesem Zweck auf qualitative Verfahren zurückgreift. ${ }^{45}$

\footnotetext{
${ }^{45}$ Im vorliegenden Abschnitt wurden Einsatzgebiete von Social-Media-Analysen und Marktforschung im Web 2.0 thematisiert; ein entsprechender Fokus bestimmte die Auswahl der nachgezeichneten Beiträge aus der Unmenge der dazu mittlerweile vorhandenen Literatur. Dass die Marktforschung einem Wandel unterworfen ist, nicht zuletzt aufgrund des Web 2.0 und sozialer Medien, sollte deutlich geworden sein. In dieser Studie bleibt kein Raum, deren Zukunft näher zu beleuchten. Anderenorts ist dies bereits geschehen, teilweise mit explizitem Bezug zu Web 2.0 und sozialen Medien. Siehe hierzu zum Beispiel: Gansser, \& Krol, 2017; Keller, Klein, \& Tuschl, 2015, 2016; Theobald, 2017, 2018.
} 
Open Access Dieses Kapitel wird unter der Creative Commons Namensnennung 4.0 International Lizenz (http://creativecommons.org/licenses/by/4.0/deed.de) veröffentlicht, welche die Nutzung, Vervielfältigung, Bearbeitung, Verbreitung und Wiedergabe in jeglichem Medium und Format erlaubt, sofern Sie den/die ursprünglichen Autor(en) und die Quelle ordnungsgemäß nennen, einen Link zur Creative Commons Lizenz beifügen und angeben, ob Änderungen vorgenommen wurden.

Die in diesem Kapitel enthaltenen Bilder und sonstiges Drittmaterial unterliegen ebenfalls der genannten Creative Commons Lizenz, sofern sich aus der Abbildungslegende nichts anderes ergibt. Sofern das betreffende Material nicht unter der genannten Creative Commons Lizenz steht und die betreffende Handlung nicht nach gesetzlichen Vorschriften erlaubt ist, ist für die oben aufgeführten Weiterverwendungen des Materials die Einwilligung des jeweiligen Rechteinhabers einzuholen.

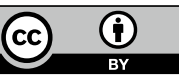

